\title{
Permanent Closure of the CFA-688 Diesel Underground Storage Tank 98CFA00260 (DEQ Facility ID\# 6-120608)
}

May 2018

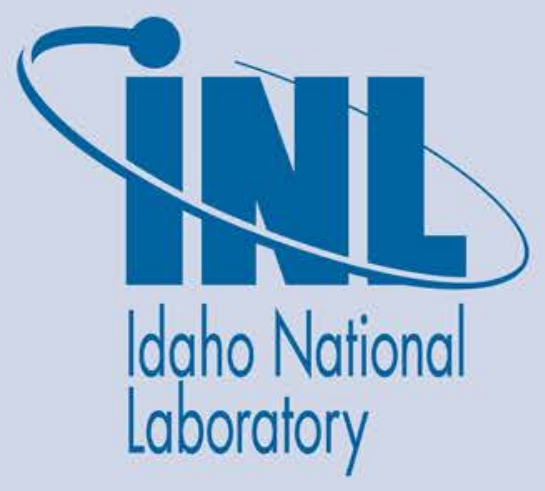

The INL is a U.S. Department of Energy National Laboratory operated by Battelle Energy Alliance 


\section{DISCLAIMER}

This information was prepared as an account of work sponsored by an agency of the U.S. Government. Neither the U.S. Government nor any agency thereof, nor any of their employees, makes any warranty, expressed or implied, or assumes any legal liability or responsibility for the accuracy, completeness, or usefulness, of any information, apparatus, product, or process disclosed, or represents that its use would not infringe privately owned rights. References herein to any specific commercial product, process, or service by trade name, trade mark, manufacturer, or otherwise, does not necessarily constitute or imply its endorsement, recommendation, or favoring by the U.S. Government or any agency thereof. The views and opinions of authors expressed herein do not necessarily state or reflect those of the U.S. Government or any agency thereof. 


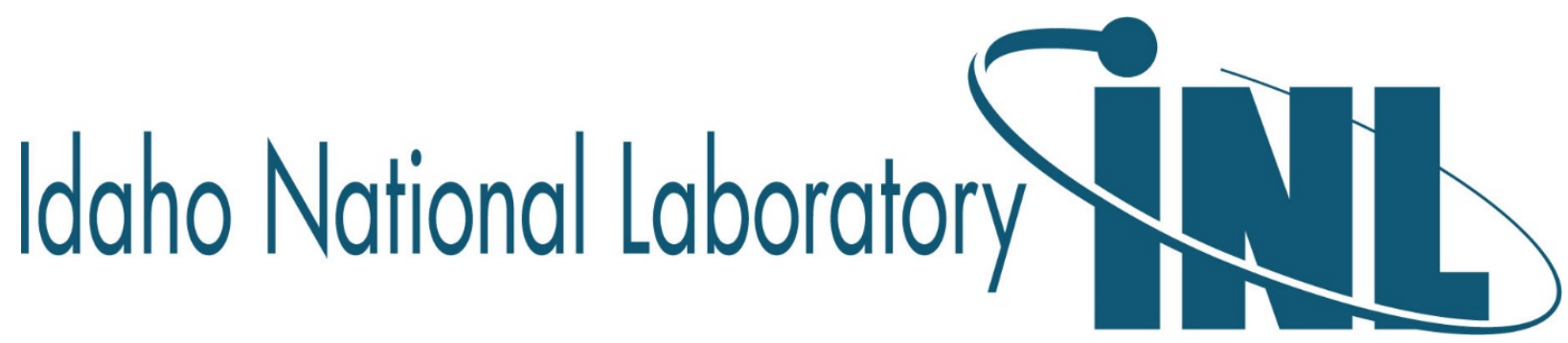

\section{Permanent Closure of the CFA-688 Diesel Underground Storage Tank 98CFA00260 (DEQ Facility ID\# 6-120608)}

May 2018

Idaho National Laboratory Idaho Falls, Idaho 83415

http://www.inl.gov

Prepared for the U.S. Department of Energy Office of Nuclear Energy Under DOE Idaho Operations Office Contract DE-AC07-05ID14517 



\section{CONTENTS}

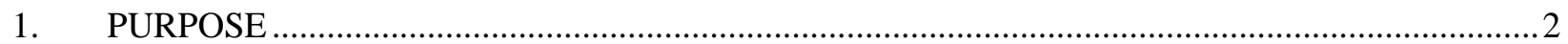

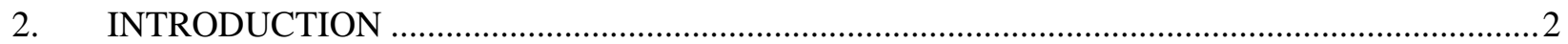

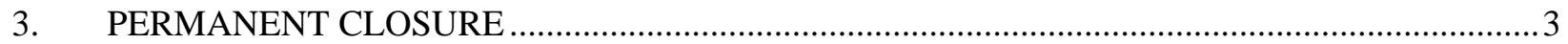

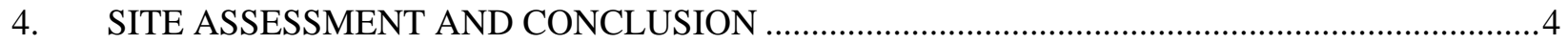

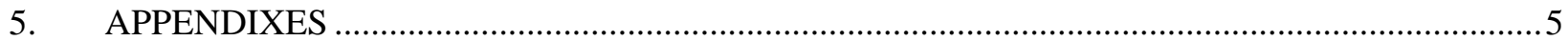

Appendix A, CCN: 241059 - 30 Day Notification for Underground Storage Tank Systems for tank Closure at CF-688........................................................................

Appendix B, CCN: 242079 - Closure Notification of CF-688 UST (DEQ Facility ID\# 6-120608).....12

Appendix C, CCN-242150 Conference call to Idaho Department of Environmental Quality -

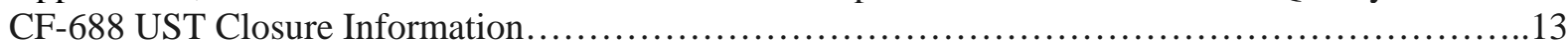

Appendix D, CCN: 242503 Preliminary Sampling Data Package Information for CF-688 Underground Storage Tank (DEQ Facility ID\# 6-120608).................................. 14

Appendix E, Sample Analytical Report TOS-231............................................15

Appendix F, Idaho Risk Evaluation Manual for Petroleum Releases - Table 2. Screening Level Concentrations for Soil, Groundwater, and Soil Vapor.......................................111

\section{FIGURES}

Figure 1. CFA-688 Underground Storage Tank Removal .................................................................

Figures 2 and 3. CFA-688 Underground Storage Tank Soil Sampling ...............................................5

\section{TABLES}

1. Key personnel. 2 


\section{PURPOSE}

This closure package documents the site assessment and petitions for permanent closure of the Idaho National Laboratory (INL) Central Facilities Area (CFA) diesel underground storage tank 98CFA00260 (DEQ Facility ID\# 6-120608), in accordance with the regulatory requirements established in 40 CFR 280.71, "Permanent Closure and Changes-In-Service”.

\section{INTRODUCTION}

The CFA diesel underground storage tank 98CFA00260 is a 2,500-gallon; double-walled, fiberglass reinforced plastic tank with fiberglass reinforced flexible plastic piping, and is located at the Idaho National Laboratory’s (INL) Central Facilities Area, CFA-688. This tank is identified under the Idaho Department of Environmental Quality (DEQ) Tank Management Plan facility identification number 6-120608 and tank number 98CFA00260.

An Idaho Department of Environmental Quality (DEQ) Underground Storage Tank (UST) inspection in September 2017 identified no violations to this tank.

The tank was installed August of 1994 to supply diesel fuel to an emergency diesel generator located inside building CF-688. The tank interstitial and sump sensors were monitored by a Gilbarco Veeder-Root TLS-350 Automatic Tank Gauge (ATG). The piping from the emergency diesel generator is fiberglass reinforced pipe.

Key personnel that were involved in this closure/decommissioning activity are listed in Table 1.

Table 1. Key personnel.

\begin{tabular}{lll}
\multicolumn{1}{c}{ Organization/Title } & \multicolumn{1}{c}{ Name } & \multicolumn{1}{c}{ Responsibilities } \\
\hline BEA Project Manager & Jeremy Bishop & Project execution and completion \\
\hline BEA Project Supervisor & Eric Walker & Project execution and completion \\
CFA Facility Manager & Bryan Crofts & Manage/approve facility activities \\
BEA Environmental Compliance & Bradley Griffith/ George & Coordinate UST closure activity
\end{tabular}




\section{PERMANENT CLOSURE}

In accordance with 40 CFR 280.71(a), a 30-day closure notification was mailed on August 30, 2017, (Appendix A, CCN 241059) notifying Idaho DEQ of INL's intent to permanently close the CFA-688 tank 98CFA00260 diesel underground storage tank (DEQ ID\# 6-120608). Michael Summers (DEQ) was contacted regarding a sampling and analysis plan. Michael stated that a sampling and analysis plan would not be required.

On February 12, 2018, a conference call was placed to Michael Summers at the Idaho DEQ Idaho Falls Office, informing him of INL's intent to remove the UST tentatively on February 19, 2018 and to identify if DEQ wanted be present during any part of the removal process. Michael stated that DEQ wanted to inspect the fill port when the asphalt and concrete was removed from around the fill port. Michael also stated that he wanted to be present during the tank removal and sampling process. It was stated that the INL would contact him when an exact date was confirmed or to any delays. Michael also e-mailed two documents for use in sampling process: Waste Management and Remediation Division Statewide Generic Quality Assurance Project Plan and Fact Sheet: How DEQ Evaluates Sample Collection and Data Analysis for UST Closures and Release Investigations (Appendix B - CCN 242150).

In preparation for demolition and permanent tank closure, the remaining fuel was removed from the tank on March 19, 2018 by Clean Harbors to as low as reasonably achievable (less than 1 inch of fuel).

In preparation for permanent closure in accordance with 40 CFR 280.71, on March 22, 2018 the UST at CF-688 was removed with Michael Summers (DEQ) in attendance. INL's Environmental Monitoring personnel collected two soil samples from under the tank (one at fill port and one at opposite end of tank) and one sample under the fuel supply line in the area identified by Michael Summers. Soil samples were sent to GEL Laboratories LLC in Charleston, South Carolina for analysis. Laboratory analysis was requested for Chemicals of Interest for Various Petroleum Products (diesel) as identified in IDAPA 58.01.24.800.01 table 1, with laboratory detection limits for the Residential Use Screening Levels in table 2. The sampling collection and handling process adhered to the Waste Management and Remediation Division Statewide Generic Quality Assurance Project Plan.

On April 4, 2018, Jill Lundell from INL Environmental Monitoring e-mailed a preliminary sampling data package received from GEL Laboratories LLC. The preliminary sampling data showed that the screening levels for all of the Chemicals of Interest for Various Petroleum Products (diesel) as identified in IDAPA 58.01.24.800.01 table 1, were reported as non-detectable with laboratory detection limits below the Residential Use Screening Levels in table 2.

On April 10, 2018, preliminary sampling data results from the analysis received from GEL Laboratories LLC was e-mailed to Michael Summers. The e-mail also stated when the final analysis data package is received, a site assessment closure report for the underground storage tank removal would be submitted to the Department of Environmental Quality for final closure (Appendix C - CCN 242150).

On April 17, 2018, the INL received a final Report from GEL Laboratories LLC for the CF-688 diesel tank and piping sampling (Appendix E). The INL compared the analysis to the Idaho Risk Evaluation Manual for Petroleum Releases, Table 2 - Screening Level Concentrations for Soil and identified that the concentrations for the chemicals of interest were below the DEQ identified screening levels for these analytes. 


\section{SITE ASSESSMENT AND CONCLUSION}

This site assessment was performed in accordance with IDAPA 58.01.24.200, "Risk Evaluation Process.” A screening evaluation was performed according to the chemicals of interest for diesel fuel found in the Idaho Risk Evaluation Manual for Petroleum Releases, Table 2 - Screening Level Concentrations for Soil, Groundwater, and Soil Vapor.

Per 40 CFR 280.71 "Permanent Closure and Changes-In-Service” all liquids and accumulated sludge was removed from the UST.

Sample results were received on April 17, 2018, and were compared to the Idaho Risk Evaluation Manual for Petroleum Releases, Table 2 - Screening Level Concentrations for Soil, Groundwater, and Soil Vapor maximum media-specific (soil) petroleum contaminant concentrations. The concentrations for the chemicals of interest are below the DEQ identified screening levels for these analytes.

According to IDAPA 58.01.24.200.01.c., "if the maximum media-specific petroleum contaminant concentrations at the site do not exceed the screening levels, the owner and/or operator may petition for site closure, subject to other Department regulatory obligations”. This site assessment meets the requirements of IDAPA 58.01.24.200. The INL is petitioning the DEQ for site closure of the CFA-688 diesel tank 98CFA00260 (DEQ ID\# 6-120608).

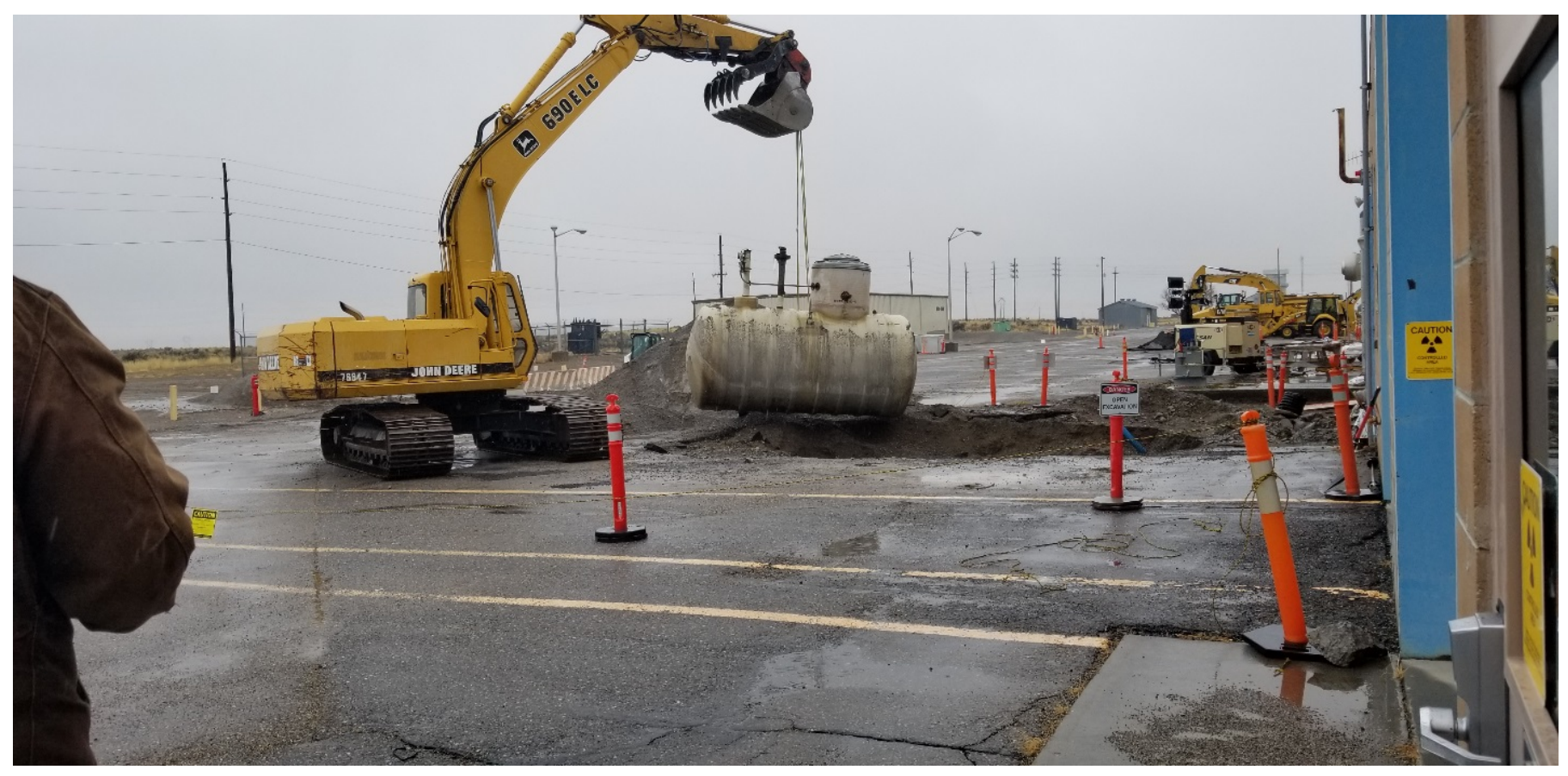

Figure 1. CFA-688 Underground Storage Tank Removal 

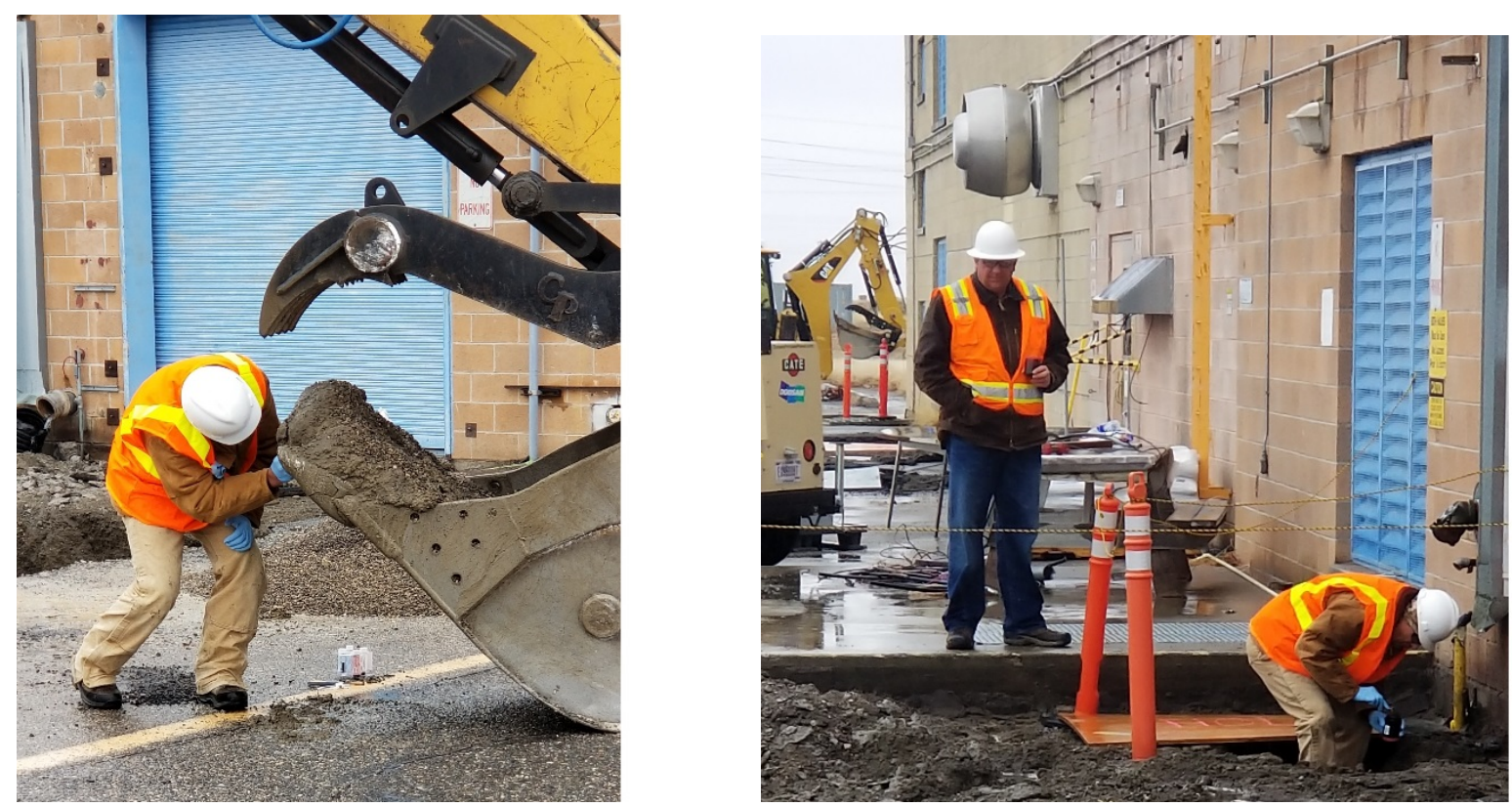

Figures 2 and 3. CFA-688 Underground Storage Tank Soil Sampling

\section{APPENDIXES}

Appendix A, CCN: 241059 - 30 Day Notification for Underground Storage Tank Systems for tank Closure at CF-688

Appendix B, CCN: 242079 - Closure Notification of CF-688 UST (DEQ Facility ID\# 6-120608)

Appendix C, CCN-242150 Conference call to Idaho Department of Environmental Quality - CF-688 UST Closure Information

Appendix D, CCN: 242503 Preliminary Sampling Data Package Information for CF-688 underground storage tank (DEQ ID\# 6-120608)

Appendix E, Sample Analytical Report TOS-231

Appendix F, Idaho Risk Evaluation Manual for Petroleum Releases - Table 2. Screening Level Concentrations for Soil, Groundwater, and Soil Vapor 


\section{Appendix A, Notification of Closure (CCN 241059)}

Nisson, Kerry L <kerry.nisson@inl.gov>

Ieroro Necional lebortion

\section{CCN: 241059 - 30 Day Notification for Underground Storage Tank Systems for tank closure at CF-688 \\ 1 message}

Nisson, Kerry L <kerry.nisson@inl.gov>

Wed, Aug 30, 2017 at 10:29 AM

To: Michael.Summers@deq.idaho.gov

Cc: Timothy A Miller <Timothy.Miller@inl.gov>, Kent Miller <kent.miller@inl.gov>, James F Graham

<James.Graham@inl.gov>, Bradley K Griffith <bradley.griffith@inl.gov>, ENVIRONMENTAL CORRESPONDENCE ServiceID <envaff@inl.gov>, BEA CORRESPONDENCE CONTROL ServicelD <beacc@inl.gov>, Jason Sturm <STURMJR@id.doe.gov>, Jeremy K Bishop <jeremy.bishop@inl.gov>

Michael

I previously sent you 30 Day Notification for Underground Storage Tank Systems for four tank closures that the Idaho National Laboratory (INL) has intended to remove this fiscal year. The tanks are:

. DEQ Facility Identification Number 6-120612 - Tank ID\# 98CFA00057 - 15,000 diesel located at Central Facilities building CF-608,

- DEQ Facility Identification Number 6-120613 - Tank ID\# 98CFA00061 - 12,000 diesel located at Central Facilities building CF-609,

- DEQ Facility Identification Number 6-120615 - Tank ID\# 99ANL00011 - 2,500 unleaded located at the Materials and Fuels Complex MFC-783, and

- DEQ Facility Identification Number 6-120615 - Tank ID\# 99ANL00012 - 2,500 diesel located at the Materials and Fuels Complex MFC-783.

Due to funding issues, these four tanks will not be removed this fiscal year. The INL has plans to remove these tanks next fiscal year, if funding is available.

The INL is performing a decommissioning and demolition (D\&D) of building CF-688 at the Central Facilities Area. This building has 2,500 gallon underground storage tank (UST) that supplied an emergency diesel generator. As part of the facility D\&D, the UST will be removed. This is tentatively scheduled for October. Attached is the 30 Day Notification for Underground Storage Tank Systems for tank closure, DEQ Facility Identification Number 6-120608 - Tank ID\# 98CFA00260.

Kerry L. Nisson

Nuclear Operations Environmental Support - UST TPOC

Office (208) 533-7102

Cell (208) 569-4721

email: kerry.nisson@inl.gov

Materials and Fuels Complex - Mail Stop 6134

\footnotetext{
CFA-688 UST-notification-form closure.pdf

$533 \mathrm{~K}$
} 
DEQ Version of EPA 7530-1 (Revised 3/2017)

\begin{tabular}{|c|c|}
\hline NOTIFICATION FOR UNDERGROUND STORAGE TANK SYSTEMS & Facility ID \\
\hline Idaho Department of Environmental Quality, 1410 N Hilton, Boise ID 83706 & $\underline{6-120608}$ \\
\hline
\end{tabular}

INSTRUCTIONS - See additional instructions on page 6

Please type or use ink. This form must be completed for each location containing underground storage tanks. If more than five (5) tanks are owned at this location, photocopy the following sheets, and attach continuation sheets to the form (pages 3,4 , \& 5)

Notification is required by law for all underground storage tanks (USTs)

storing regulated substances that are brought into use after May 8,1986 , or USTs in the ground as of May 8,1986, that have stored regulated substances at any time since January 1,1974 . The information requested is required by Section 9002 of the Resource Conservation and Recovery Act (RCRA), as amended.

The primary purpose of this notification form is to provide information about the installation, existence, changes to, and closure of USTs that store or have stored petroleum or hazardous substances. The information you provide will be based on reasonably available records, or in the absence of such records, your knowledge or recollection.

Who must notify? Unless exempted, owners of USTs that store or will store regulated substances must notify $D E Q$.

1. Owner means -

a) in the case of an UST in use on November 8,1984 , or brought into use after that date, any person who owns an underground storage tank used for the storage, use, or dispensing of regulated substances

b) in the case of an UST in use before November 8,1984 , but no longer in use on that date, any person who owned such tank immediately before the discontinuation of its use

c) in the case of a new installation on or after April 2, 2008, any person who will install an underground storage tank system

d) in the case of an underground storage tank closure, any person who will remove or close in place such tank

e) in the case of changes or updates, any person who will make a change to an UST system

What tanks are included? Underground storage tank is defined as any one or combination of tanks that is used to contain an accumulation of "regulated substances," and whose volume (including connected underground piping) is $10 \%$ or more beneath the ground.

What tanks are excluded?

1.Tanks with a capacity of 110 gallons or less

2. Farm or residential tanks of 1,100 gallons or less capacity used for storing

motor fuel for noncommercial purposes

3. Tanks used for storing heating oil for consumptive use on the premises

where stored

4. Septic tanks

5. Certain pipeline facilities regulated under chapters 601 and 603 of Title 49

6. Surface impoundments, pits, ponds, or lagoons

7. Stormwater or wastewater collection systems

8. Flow-through process tanks
9. Liquid traps or associated gathering lines directly related to oil or gas production and gathering operations

10. Tanks on or above the floor of underground areas, such as basements or tunnels

11. Wastewater treatment tanks

12. UST systems containing radioactive material that are regulated under the Atomic Energy Act of 1954

13. UST systems that are part of an emergency generator system at nuclear power generation facilities regulated by the Nuclear

Regulatory Commission under 10 CFR Part 50

What substances are covered? The notification requirements apply to USTs containing petroleum or certain hazardous substances. Petroleum includes gasoline, used oil, diese fuel, crude oil, or any fraction thereof which is liquid at standard conditions of temperature and pressure ( 60 degrees Fahrenheit and 14.7 pounds per square inch absolute). Hazardous substances are those found in section 101 (14) of the Comprehensive Environmental Response, Compensation and Liability Act of 1980, with the exception of those substances regulated as hazardous waste under Subtitle C of the Resource Conservation and Recovery Act.

Where to notify? Send completed forms to:

UST Coordinator
Idaho Department of Environmental Quality
1410 N. Hilton
Boise, ID 83706 Telephone: (208) 373-0502

When to notify? Owners of underground storage tank systems that are still in the ground must notify immediately. Owners who bring USTs into use after May 8,1986, must notify within 30 days of bringing the tanks into use. Owners who will install an UST system must notify 30 days prior to the installation. Owners who will replace $50 \%$ of piping connected to a single underground storage tank must notify 24 hours prior to the replacement. Owners who will close an UST must notify 30 days prior to the closure. Owners who have closed an UST must notify and indicate the date of closure. New owners must notify within 30 days of ownership.

Penalties: Any owner who knowingly fails to notify or submits false information shall be subject to a clvil penalty.

Name U.S. Department of Energy, Idaho Operations Office (DOE-ID)

Mailing Address 1955 Fremont Avenue

City Idaho Falls

State Idaho

ZIP Code 83401

County Bonneville

Phone Number (With Area Code) (208) 526-2493

Email sturmir@id.doe.gov

(If same as Section I, mark box here $\square$ ) Name U.S. Department of Energy, Idaho Operations Office (DOE-ID) Street Address (no PO Box) Central Facilities Area

City Scoville

State Idaho

ZIP Code 83415

County Butte 


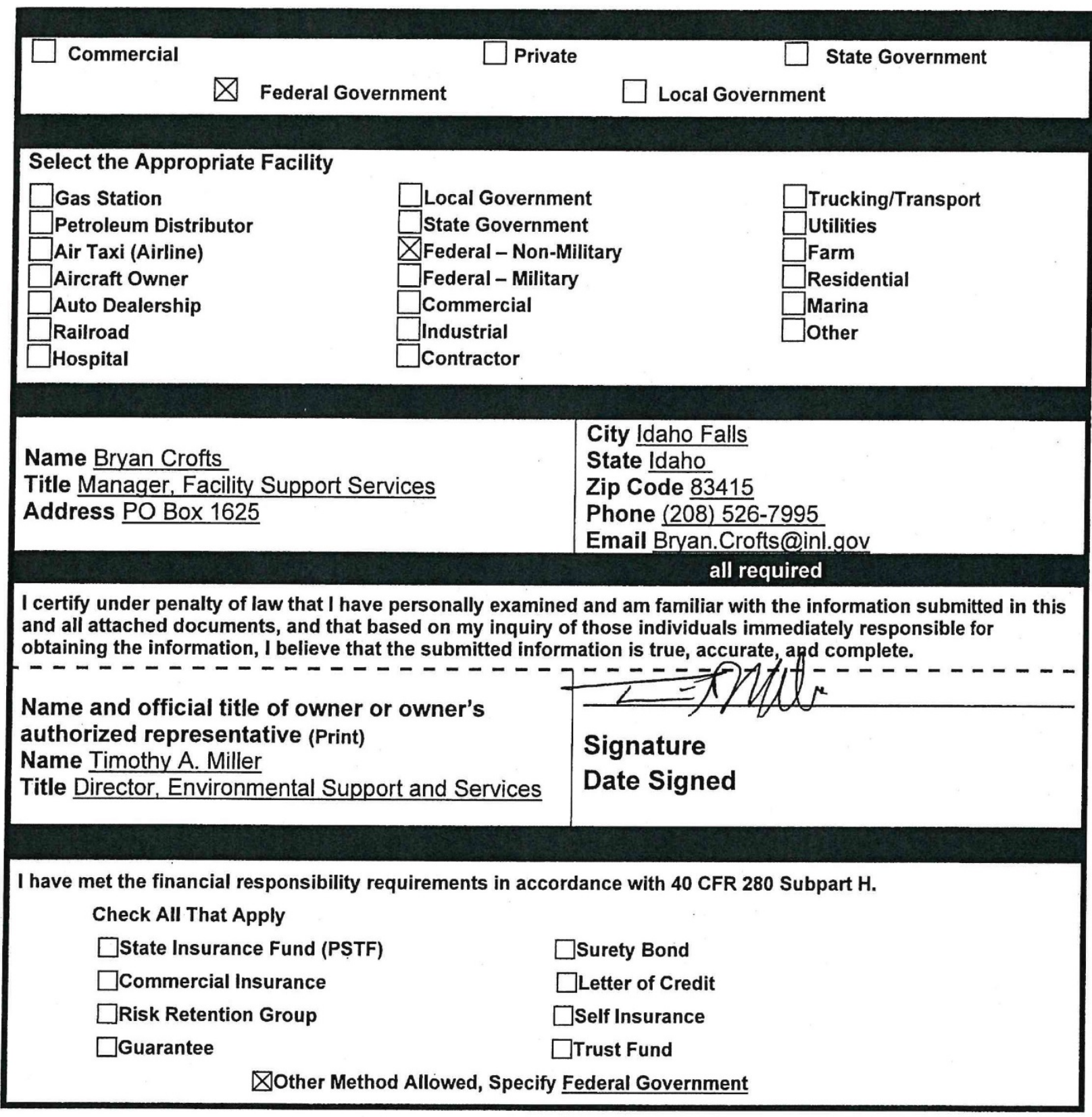




\begin{tabular}{|c|c|c|c|c|c|}
\hline IDENTIFICATION NUMBER & $\begin{array}{c}\text { Tank No. } \\
\text { 98CFA00260 }\end{array}$ & Tank No. & Tank No. & Tank No. & Tank No. \\
\hline \multicolumn{6}{|c|}{ A. 30-day Tank and Piping Installation/24-hr Piping Replacement Notifications (see page 7) } \\
\hline \multicolumn{6}{|c|}{\begin{tabular}{|r|} 
When will tank be installed or replaced? \\
When will piping be installed or
\end{tabular}} \\
\hline $\begin{array}{r}\text { When will piping be installed or } \\
\text { replaced? }\end{array}$ & & & & & \\
\hline \multicolumn{6}{|l|}{ B. 30-day Notice of Closures (see page 7) } \\
\hline When will tank be closed? & Oct. 2, 2017 & & & & \\
\hline \multicolumn{6}{|l|}{ Date tank was last used? } \\
\hline \multicolumn{6}{|l|}{ Closure to be performed by: } \\
\hline \multirow{2}{*}{\multicolumn{6}{|c|}{$\begin{array}{l}\text { Company Site Supervisor: } \\
\text { Phone: }\end{array}$}} \\
\hline & & & & & \\
\hline \multirow{2}{*}{\multicolumn{6}{|c|}{$\frac{\text { IDENTIFICATION NUMBER }}{\text { A. Type of Tank (check all that apply) }}$}} \\
\hline A. Type of Tank (check all that apply) & & & & & \\
\hline$\square$ Compartment & \multicolumn{2}{|c|}{ 凶Emergency Generator } & & \multicolumn{2}{|c|}{$\square$ Airport Fuel Hydrant } \\
\hline$\square$ Manifold & \multicolumn{2}{|c|}{$\square$ Field-Constructed } & & & \\
\hline \multicolumn{6}{|l|}{ B. Status of Tank } \\
\hline Currently In Use & No & Select & Select & Select & Select \\
\hline \multirow{2}{*}{\multicolumn{6}{|c|}{$\begin{array}{r}\text { Temporarily Out of Use } \\
\text { (Complete Section X, estimated date last used) } \\
\text { Permanently Out of Use }\end{array}$}} \\
\hline & Select & Select & Select & Select & Select \\
\hline Date of Installation & $8 / 94$ & & & & \\
\hline Total Capacity (gallons) & 2500 & & & & \\
\hline Substance Currently or Last Stored & Diesel & Select & Select & Select & Select \\
\hline \multicolumn{6}{|l|}{ CERCLA Name or CAS \# (if hazardous) } \\
\hline \multicolumn{6}{|l|}{ C. Tank Construction (Mark all that apply) } \\
\hline Fiberglass Reinforced Plastic & 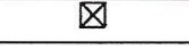 & $\square$ & $\square$ & $\square$ & $\square$ \\
\hline Cathodically Protected Steel (STIP-3) & $\square$ & $\square$ & $\square$ & $\square$ & $\square$ \\
\hline $\begin{array}{r}\text { Cathodically Protected Steel } \\
\text { (Impressed Current) }\end{array}$ & $\square$ & $\square$ & $\square$ & $\square$ & $\square$ \\
\hline Composite (Steel with Fiberglass) & $\square$ & $\square$ & $\square$ & $\square$ & $\square$ \\
\hline Asphalt Coated or Bare Steel & $\square$ & $\square$ & $\square$ & $\square$ & $\square$ \\
\hline Concrete & $\square$ & $\square$ & $\square$ & $\square$ & $\square$ \\
\hline Double-Walled & 凶 & $\square$ & $\square$ & $\square$ & $\square$ \\
\hline Lined Interior & $\square$ & $\square$ & $\square$ & $\square$ & $\square$ \\
\hline Polyethylene Tank Jacket & $\square$ & $\square$ & $\square$ & $\square$ & $\square$ \\
\hline Unknown & $\square$ & $\square$ & $\square$ & $\square$ & $\square$ \\
\hline Other, Please Specify & $\square$ & $\square$ & $\square$ & $\square$ & $\square$ \\
\hline & No & Select & Select & Select & Select \\
\hline \multicolumn{6}{|l|}{ Has tank been repaired? (circle one) } \\
\hline \multicolumn{6}{|l|}{ D. Spill and Overfill Protection } \\
\hline $\begin{array}{r}\text { Overfill Device Installed? } \\
\text { (Alarm, Flapper) }\end{array}$ & Alarm & Select & Select & Select & Select \\
\hline
\end{tabular}

Page 3 


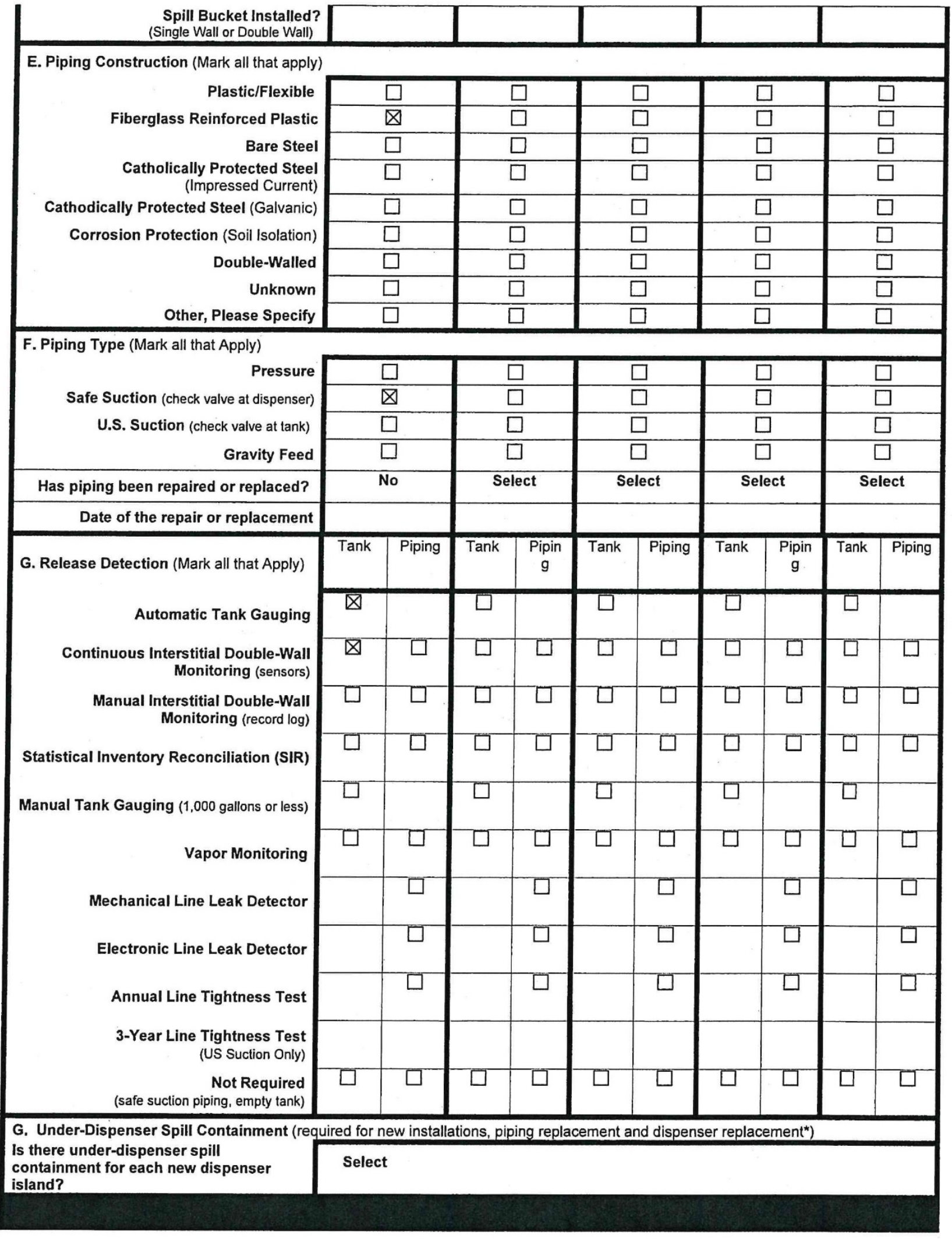

Page 4 


\begin{tabular}{|c|c|c|c|c|c|}
\hline TANK IDENTIFICATION NUMBER & Tank No. & Tank No. & Tank No. & Tank No. & Tank No. \\
\hline \multicolumn{6}{|l|}{ Closing of Tank } \\
\hline \multirow{2}{*}{$\begin{array}{c}\text { Tank Was Removed From Ground } \\
\text { Tank Was Closed In Ground }\end{array}$} & $\square$ & $\square$ & $\square$ & $\square$ & $\square$ \\
\hline & $\square$ & $\square$ & $\square$ & $\square$ & $\square$ \\
\hline \multicolumn{6}{|l|}{ Estimated Date Last Used } \\
\hline $\begin{array}{l}\text { Tank Filled With Inert Material } \\
\text { (indicate material - sand, concrete) }\end{array}$ & & & & & \\
\hline $\begin{array}{c}\text { Change in Service } \\
\text { (No longer holds a regulated substance) }\end{array}$ & $\square$ & $\square$ & $\square$ & $\square$ & $\square$ \\
\hline $\begin{array}{r}\text { Site Assessment Completed } \\
\text { (samples taken) }\end{array}$ & Select & Select & Select & Select & Select \\
\hline Evidence of a Release Detected? & Select & Select & Select & Select & Select \\
\hline Release Reported to DEQ? & Select & Select & Select & Select & Select \\
\hline Date Release Reported to DEQ & & & & & \\
\hline
\end{tabular}

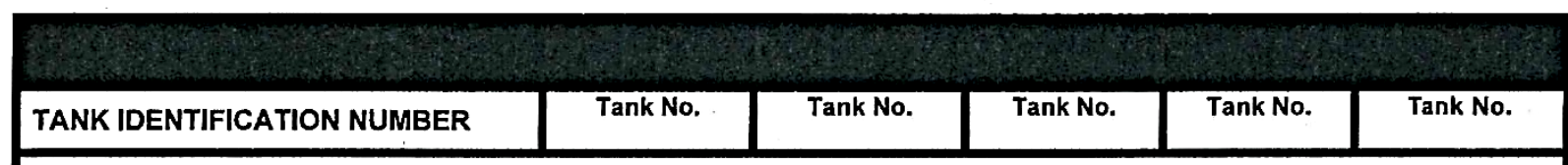

A. Installation (Mark all that apply)

\begin{tabular}{|c|c|c|c|c|c|}
\hline $\begin{array}{r}\text { Installer certified by tank and piping } \\
\text { manufacturers }\end{array}$ & $\square$ & $\square$ & $\square$ & $\square$ & $\square$ \\
\hline Installer certified or licensed by a State & $\square$ & $\square$ & $\square$ & $\square$ & $\square$ \\
\hline $\begin{array}{r}\text { Installation is inspected by a registered } \\
\text { engineer }\end{array}$ & $\square$ & $\square$ & $\square$ & $\square$ & $\square$ \\
\hline Installation inspected by DEQ & $\square$ & $\square$ & $\square$ & $\square$ & $\square$ \\
\hline $\begin{array}{r}\text { Manufacturer's installation checklists } \\
\text { have been completed }\end{array}$ & $\square$ & $\square$ & $\square$ & $\square$ & $\square$ \\
\hline
\end{tabular}

Note: The installer must complete this section only if work on your UST system has taken place since December 22, 1988.

OATH: I certify the information concerning installation is true to the best of my belief and knowledge.

Installation Company

Address:

Installer Name

Phone

Signature

Date

"When a dispenser is replaced and any equipment necessary to connect the dispenser to the underground storage tank system under the dispenser is installed; under dispenser containment is required. 


\section{Appendix B \\ CCN-242150 Conference call to Idaho Department of Environmental Quality - CF-688 UST Closure Information}

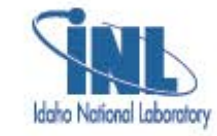

Nisson, Kerry L <kerry.niss on@inl.gov>

CCN: 242079 - Closure Notification of CF-688 UST (DEQ Facility ID\# 6-120608)

1 message

Nisson, Kerry $\mathrm{L}<$ kerry.nisson@inl.gov>

To: Michael.Summers@deq.idaho.gov

Cc: Bradley K Griffith <bradley.griffith@inl.gov>, "Krauszer II, George J" <george krausze rii@inl. gov>, James F Graham <James.Graham@inl.gov>, Kent Miller <kent.miller@inl.gov>, Timothy A Miller <T imothy.Miller@inl.gov>, Jason Sturm <STURMR@id.doe.gov>

Michael

In an email dated August 30, 2018 (ref. CCN: 241059 - 30 D ay N otification for Underground Stor age Tank Systems for tank closure at CF-688), I sent you the DEQ form Notification for Underground Starage Tank Systems for the closure of the UST atCF-688 (DEQ Facility ID\# 6-120608). The project projected that the tank would be removed in October 2017. Due to various factors, that did not happen

The project has now resumed and will have the rem airing fuel in the tark removed on February 13, 2018. The project anticipates removal of the tank during the week of $\mathrm{F}$ ebruary 19-26, 2018.

I know that the DEQ wants to be there when the tark is removed and for the sampling requirements once the tarik is removed.

Is there any additional time that you want to be present while the tank and piping are being uncovered?

I have attached the Notification for Underground Storage Tark Systems that was previously submitted in August, 2017.

I will keep you inf ormed as the project progresses and provide you the recuired 48 hour notice before the UST is removed.

Kerry L. Nisson

Nuclear Operations Environmental Support - UST TPOC

Office (208) 533-7102

Cell (208) 569-4721

em ail: kerry nisson@inl.gov

Materials and Fuels Complex - Mril Stop 6134

7 CFA-688 UST-notification-form closure.pdf

$533 \mathrm{~K}$ 


\title{
Appendix C \\ CCN-242150 Conference call to Idaho Department of Environmental Quality - CF-688 UST Closure Information
}

\author{
VN1 \\ Nisson, Kerry L <kerry.niss on@inl.gov \\ CCN-242150 Conference call to Idaho Department of Environmental Quality - CF-688 \\ UST Closure Information \\ 1 message \\ Nisson, Kerry $\mathrm{L}<$ kerry.nisson@inl.gov> \\ Mon, Feb 12,2018 at $11: 31$ AM \\ To: Kent Miller <kent.miller@inl.gov> \\ Cc: Bradley K Griffith <bradley.griffith@inl.gov>, "Krauszer II, George J" <george krauszerii@inl. gov>, James F Graham \\ $<$ James.Graham@inl.gov>, Jason Sturm <STURMJR@id.doe.gov>, ENVIRONMENTAL CORRESPONDENCE ServiceID \\ <envaff@inl.gov>,BEA CORRESPONDENCE CONTROL ServicelD <beacc@inl.gov> \\ On February 12,2018 at $\sim 07: 58$, a conference call was place to Michael Summers at the Idaho Department \\ of Environmental Quality (IDEQ). The call was placed at the request from Michael Summers [ref. attached \\ email, RE: 242079 - Closure Notification of CF-688 UST (DEQ Facility ID\# 6-120608)] to ensure that the \\ INL was meeting the DEQ's Waste Management and Remediation Division Statewide Generic Quality \\ Assurance Project Plan - for the underground storage tank closure for the removal of the 2,500 gallon \\ emergency diesel generator underground storage tank at CF-688. The conference call included Michael \\ Summers (DEQ), Jason Sturm (DOE-ID), Bradley Griffith (BEA - UST TPOC Backup), George Krauszer II \\ (BEA), and Kerry Nisson (BEA - UST TPOC).
}

Michael emailed two links to Kerry Nisson - *DEQ's Waste Management and Remediation Division Statewide Generic Quality Assurance Project Plan - Third-Party Petroleum Storage Tank Release Investigation and UST Closure and ${ }^{*}$ Change-in-Service and Idaho Department of Environmental Quality Fact Sheet: How DEQ Evaluates Sample Collection and Data Analysis for UST Closures and Release Investigations. The information in the se links needs to be followed for the sampling and analysis during the removal of the underground storage tank at CF-688 to ensure proper sample collection/handling and the sample analysis samples are being conducted according to the DEQ's standards. The email and links were distributed by email to those listed on the conference call.

Michael requested to be contacted when the asphalt and concrete was removed from around the fill port so that he could inspect this area for potential fuel leakage. Michael al so requested to be present once the tank was ready to be removed and for sampling under the tank and line.

Michael was informed that the UST removal project was tentatively scheduled for the week of February $19^{\text {th }}$.

As a side note, Michael requested that we send him an Automatic Tank Gauge (Veeder-Root monitor) tape printout showing the corrected time and date for the monitor at IF-603. The time and date on the monitor tape was found to be incorrect at the time of the three year inspection. The monitor had been repaired when an unexpected power outage damaged the circuit board. The circuit board was replaced and the monitor had to be reprogrammed. The Service Provider did not enter the current date and time when reprogramming the monitor software. The date and time reverted to the software's last update time and date. It was stated that we would send him a new tape from the monitor showing that the time and date is now current.

The conference call concluded at $\sim 08: 11$. 


\section{Appendix D \\ CCN: 242503 Preliminary Sampling Data Package Information for CF-688 Underground Storage Tank (DEQ ID\# 6-120608)}

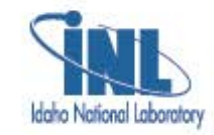

Nisson, Kerry L <kerry.niss onäinl.gov>

CCN: 242503 Preliminary Sampling Data Package Information for CF-688

underground storage tank (DEQ ID\# 6-120608)

1 message

Nisson, Kerry $L<$ kerry.nisson@inl.gov>

To: michael.summers@ deq.idaho.gov

Cc: BEA CORRESPONDENCE CONTROL ServiceID <beacc@inl. gov>, ENVIRONMENTAL CORRESPONDENCE

ServicelD <envaff@inl.gov>, Kent Miller <kent. millen@inl. gov>, James F Graham <James.Graham@inl. gov>, Bradley K

Griffith <bradley.griffith@inl.gov>, Jason Sturm <STURMUR@id.doe.gov>, "Krauszer II, George J"

<george.krauszerii@inl.gov>. Bryan P Crofts <bryan.crofts@inl.gov>,Timothy A Miller <Timothy.Miller@inl.gov>

Michael

A preliminary sampling data package for samples taken under the CF- 688 underground storage tank (DEQ ID\# 6-120608) and associated piping, show that the screening levels for all of the Chemicals of Interest for Various Petroleum Products (diesel) as identified in IDAPA 58.01.24.800.01 table 1, were reported as nondetectable and laboratory detection limits are below the Residential Use Screening Levels in table 2.

When the final analysis date package is received, a full closure report for the underground storage tank removal will be submitted to the Department of Environmental Quality for final closure.

I al so want to restate that the tanks at CF-608 (DEQ D\# 6-120612) and CF-609 (DEQ D\# 6-120613) are in temporary closure status. I am attaching a copy of the Notification for Underground Storage Tank System form for both of these two underground storage tanks that you should al ready have.

Kerry L. Nisson

Nuclear Operations Environmental Support - UST TPOC

Office (208) 533-7102

Cell (208) 569-4721

em sil: kerrynisson@inl.gov

Materials and Fuels Complex - Mri1 Stop 6134

2 attachments

园 CF-608 30 Day Notification Form Temporary Closure(signed).pdf $751 \mathrm{~K}$

团 $\begin{aligned} & \text { CF-609 } 30 \text { Day Notification Form Temporary Closure(signed).pdf } \\ & 510 \mathrm{~K}\end{aligned}$ 


\section{Appendix E \\ Sample Analytical Report \\ TOS-231 Data Package 1}

\section{GEL Laboratories LLC}

a member of The GEL Group INC

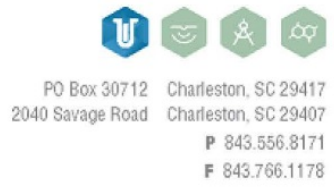

gel.com

March 30, 2018

Ms. Jill Lundell

North Wind - Portage

1075 South Utah Ave.

Suite 200

Idaho Falls, Idaho 83402

Re: Analytical for TOS-231

Work Order: 446517

Dear Ms. Lundell:

GEL Laboratories, LLC (GEL) appreciates the opportunity to provide the enclosed analytical results for the sample(s) we received on March 23, 2018. This original report has been prepared and reviewed in accordance with GEL's standard operating procedures.

Our policy is to provide high quality, personalized analytical services to enable you to meet your analytical needs on time every time. We trust that you will find everything in order and to your satisfaction. If you have any questions, please do not hesitate to call me at (843) 556-8171, ext. 4453.

Sincerely,

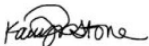

Kaitlyn Stone for

Edith Kent

Project Manager

Purchase Order: 23270002P01

Chain of Custody: 116

Enclosures 


\section{Table of Contents}

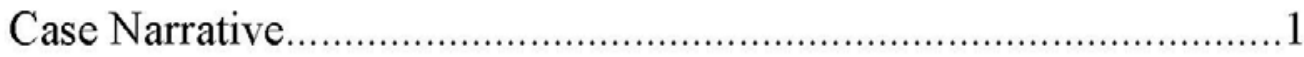

Chain of Custody and Supporting Documentation...............................4

Data Review Qualifier Definitions..................................................

Laboratory Certifications....................................................................10

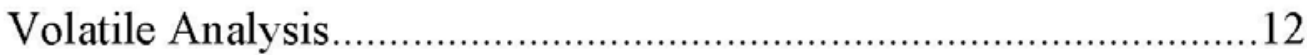

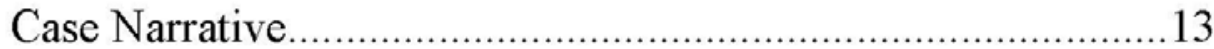

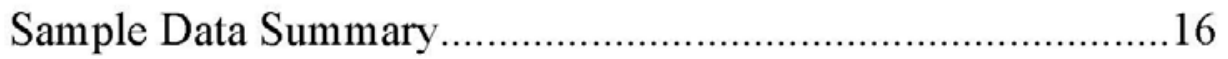

Quality Control Summary ......................................................21

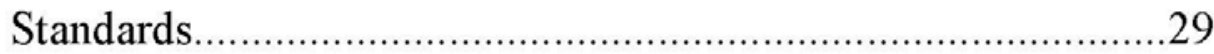

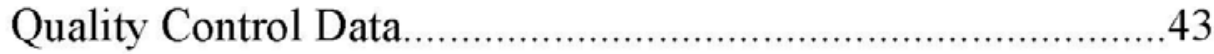

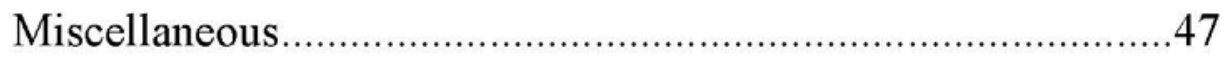

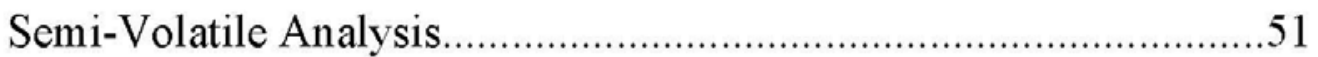

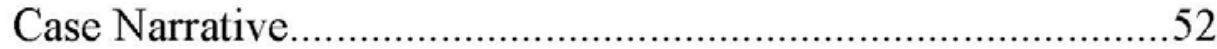

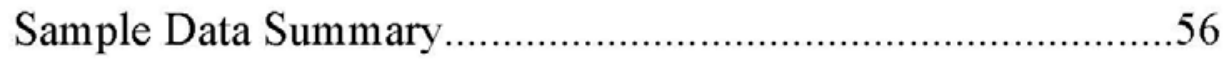

Quality Control Summary........................................................61

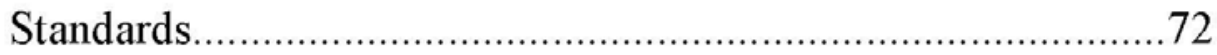

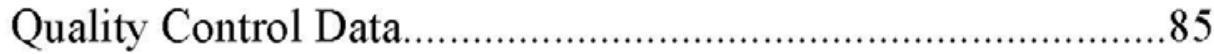

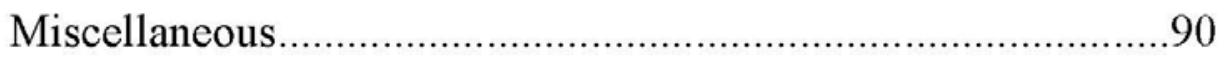




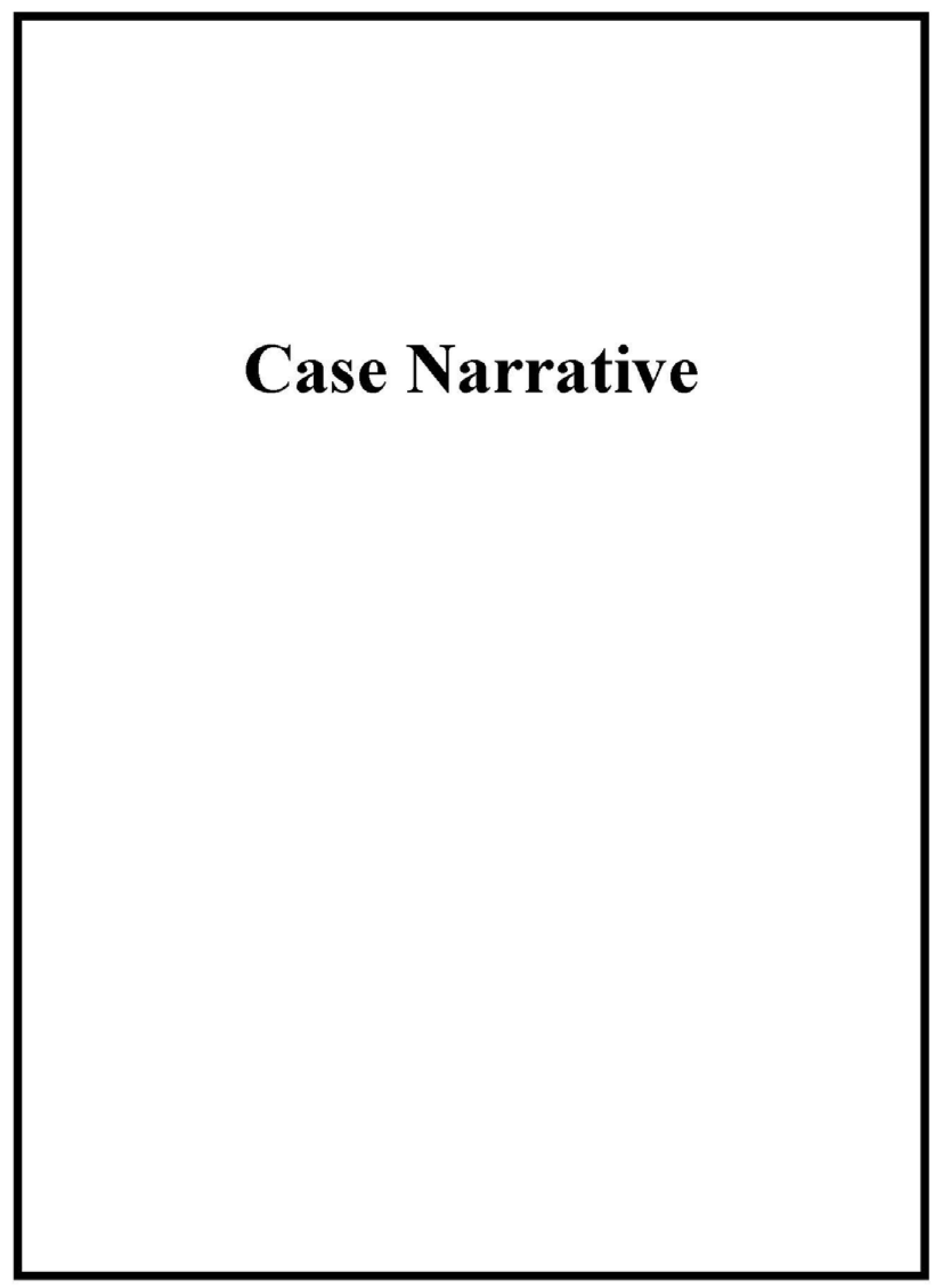

Page 1 of 94 


\section{Case Narrative \\ for \\ North Wind - Portage (8-00000013) \\ SDG: 446517}

March 30, 2018

Laboratory Identification:

GEL Laboratories LLC

2040 Savage Road

Charleston, South Carolina 29407

(843) 556-8171

$\underline{\operatorname{TOS}}$

Analytical for

Project Title

Tank Removal at CFA

Summary:

Sample Receipt The samples arrived at GEL Laboratories LLC, Charleston, South Carolina on March 23, 2018 for analysis. The samples were delivered with proper chain of custody documentation and signatures. All sample containers arrived without any visible signs of tampering or breakage. Shipping container temperatures were checked, documented, and within specifications. There are no additional comments concerning sample receipt.

Items of Note There are no additional items of note concerning this SDG.

Sample Identification The laboratory received the following sample(s):

Laboratory ID Client ID

446517001

446517001

446517002

446517002

446517003

446517003

446517004

446517004
CFA18001 - Soil Location \#1

CFA18001 - Soil Location \#1

CFA18002 - Soil Location \#2

CFA18002 - Soil Location \#2

CFA18003 - Soil Location \#3

CFA18003 - Soil Location \#3

CFA18003 - DUP - Soil Location \#3 Soil

CFA18003 - DUP - Soil Location \#3 Soil
Matrix $\underline{\text { LIC }}$

Soil SVO-A-007

Soil VOA-A-013

Soil SVO-A-007

Soil VOA-A-013

Soil SVO-A-007

Soil VOA-A-013

SVO-A-007

VOA-A-013

\section{Case Narrative}

Sample analyses were conducted using methodology as outlined in GEL Laboratories, LLC (GEL) Standard Operating Procedures. Any technical or administrative problems during analysis, data review, and reduction are contained in the analytical case narratives in the enclosed data package.

\section{GEL Laboratories uc}




\title{
Prep Methods and Prep Dates
}

\section{Method $\quad$ Run Date ID}

SW846 3541 29-MAR-2018

SW846 5035 22-MAR-2018

\section{Analysis Methods and Analysis Dates}

$\begin{array}{lr}\text { Method } & \underline{\text { Run Date ID }} \\ \text { SW846 3541/8270D SIM PAH } & \text { 29-MAR-2018 } \\ \text { SW846 3541/8270D SIM PAH } & \text { 30-MAR-2018 } \\ \text { SW846 8260B } & \text { 29-MAR-2018 }\end{array}$

\section{Certification Statement}

I certify that this data package is in compliance with the terms and conditions of SOW-0062 and any applicable TOSs for this project, both technically and for completeness, for other than the conditions detailed in this case narrative. Release of the data contained in this data package and also in any associated computer-readable data submitted has been authorized by the laboratory manager or manager's designee.

\author{
ranfostone \\ Kaitlyn Stone for \\ Edith Kent \\ Project Manager
}




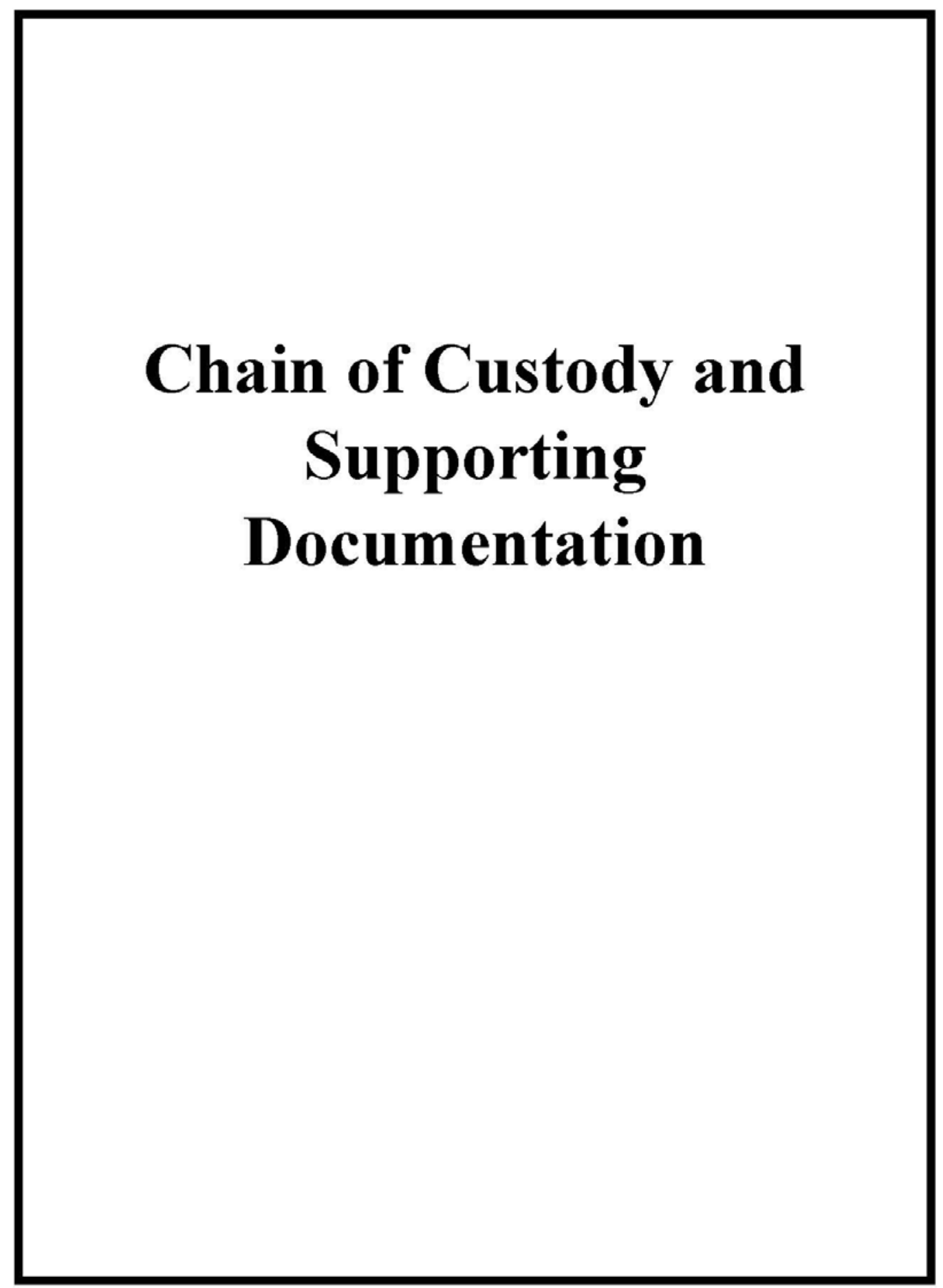

Page 4 of 94 


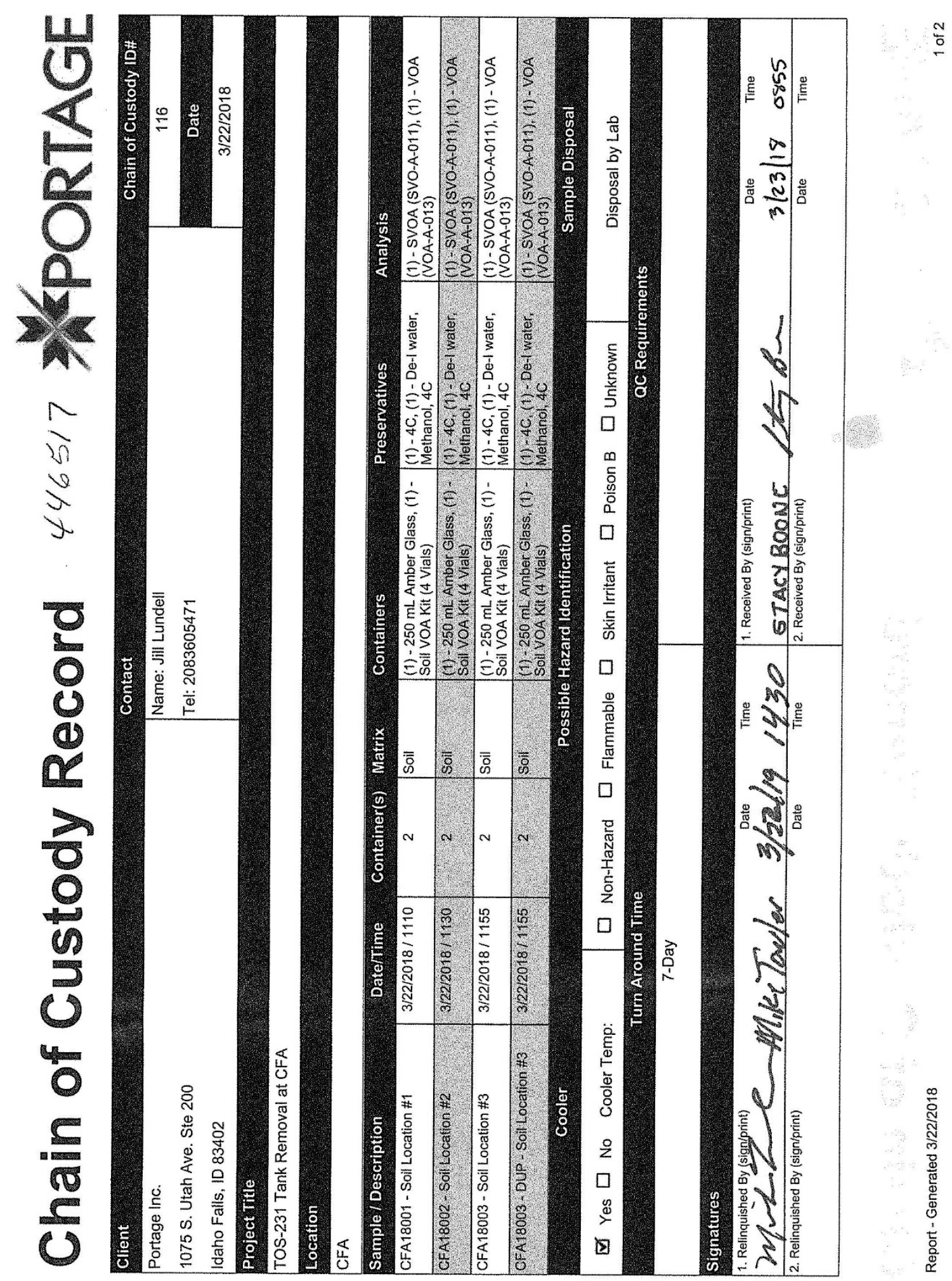

Page 5 of 94 
G피 Laboratories LC

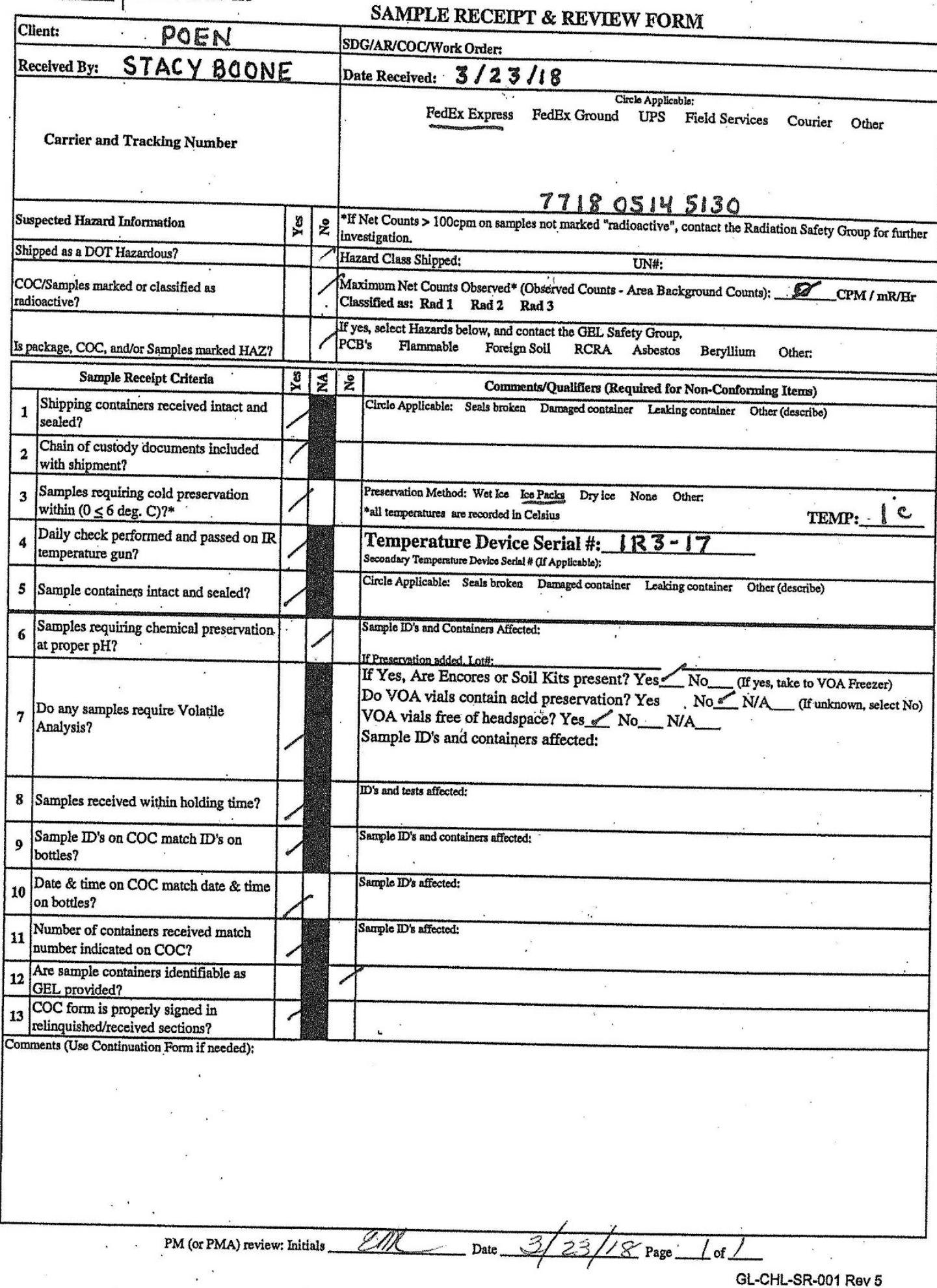

Page 6 of 94 


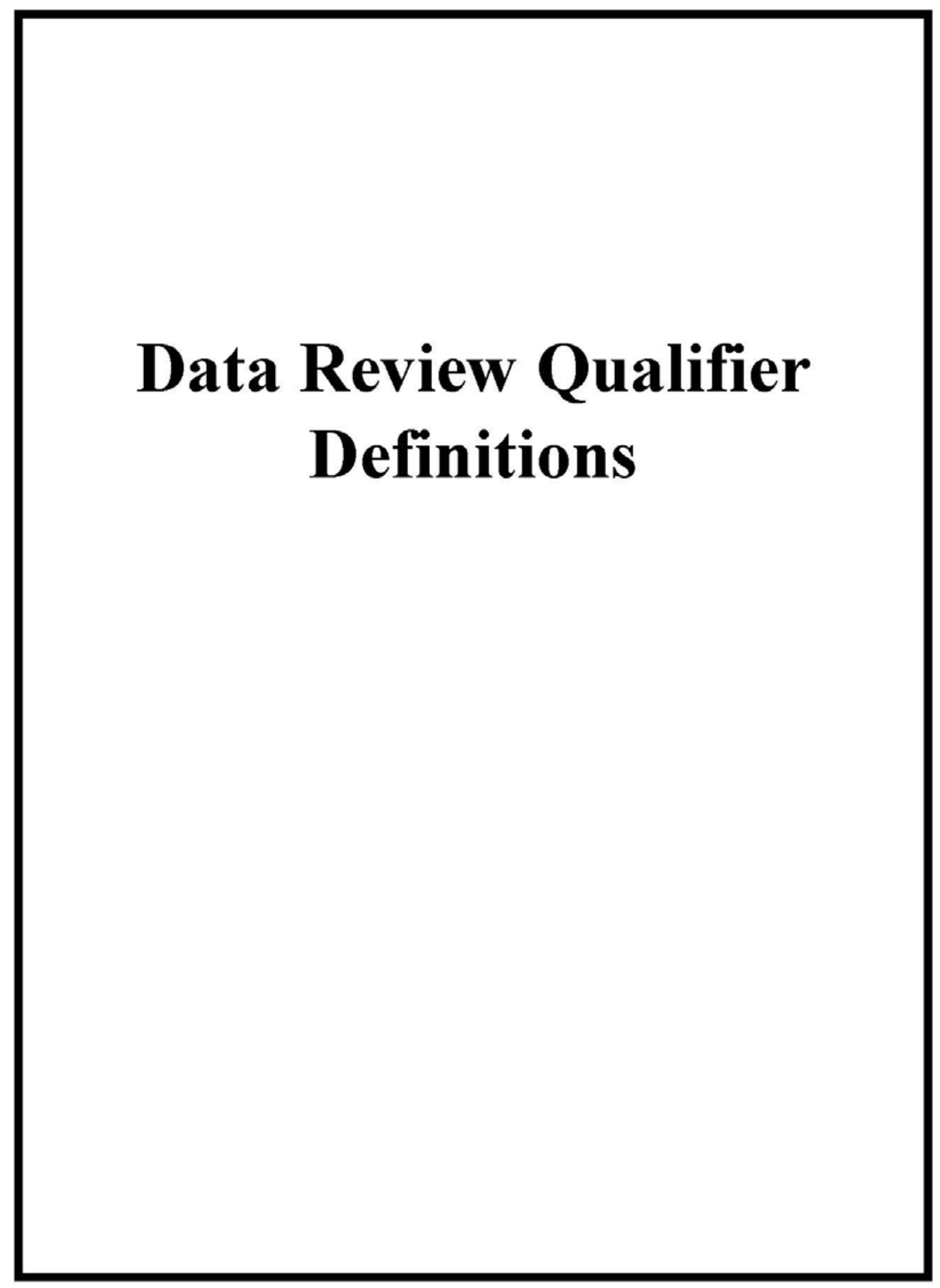

Page 7 of 94 


\section{Data Review Qualifier Definitions}

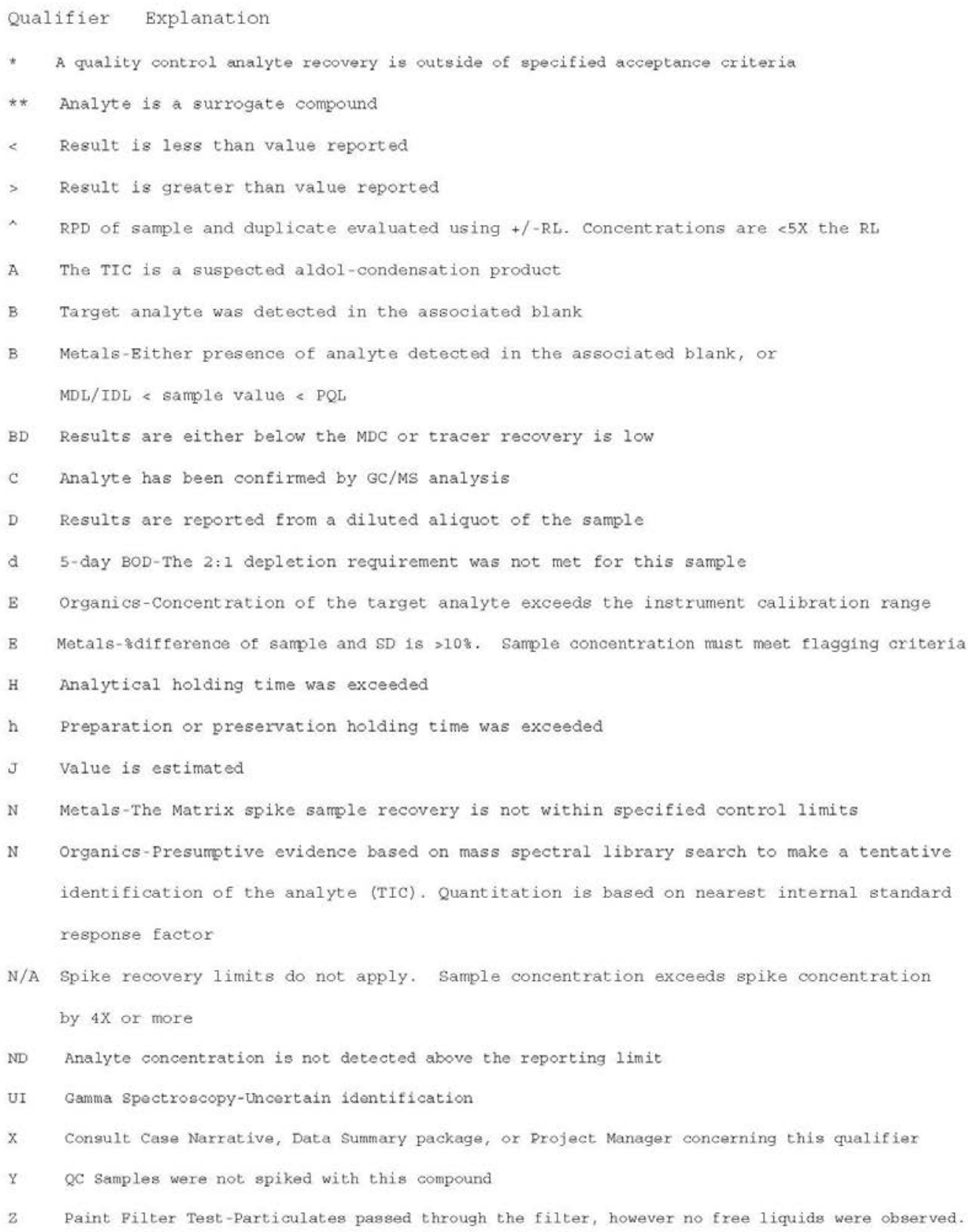

Page 8 of 94 
Organics-The concentrations between the primary and confirmation columns/detectors is $>40$ difference.

For HPLC, the difference is $>70 \%$.

U Analyte was analyzed for, but not detected above the MDL, MDA, or LOD.

Page 9 of 94 


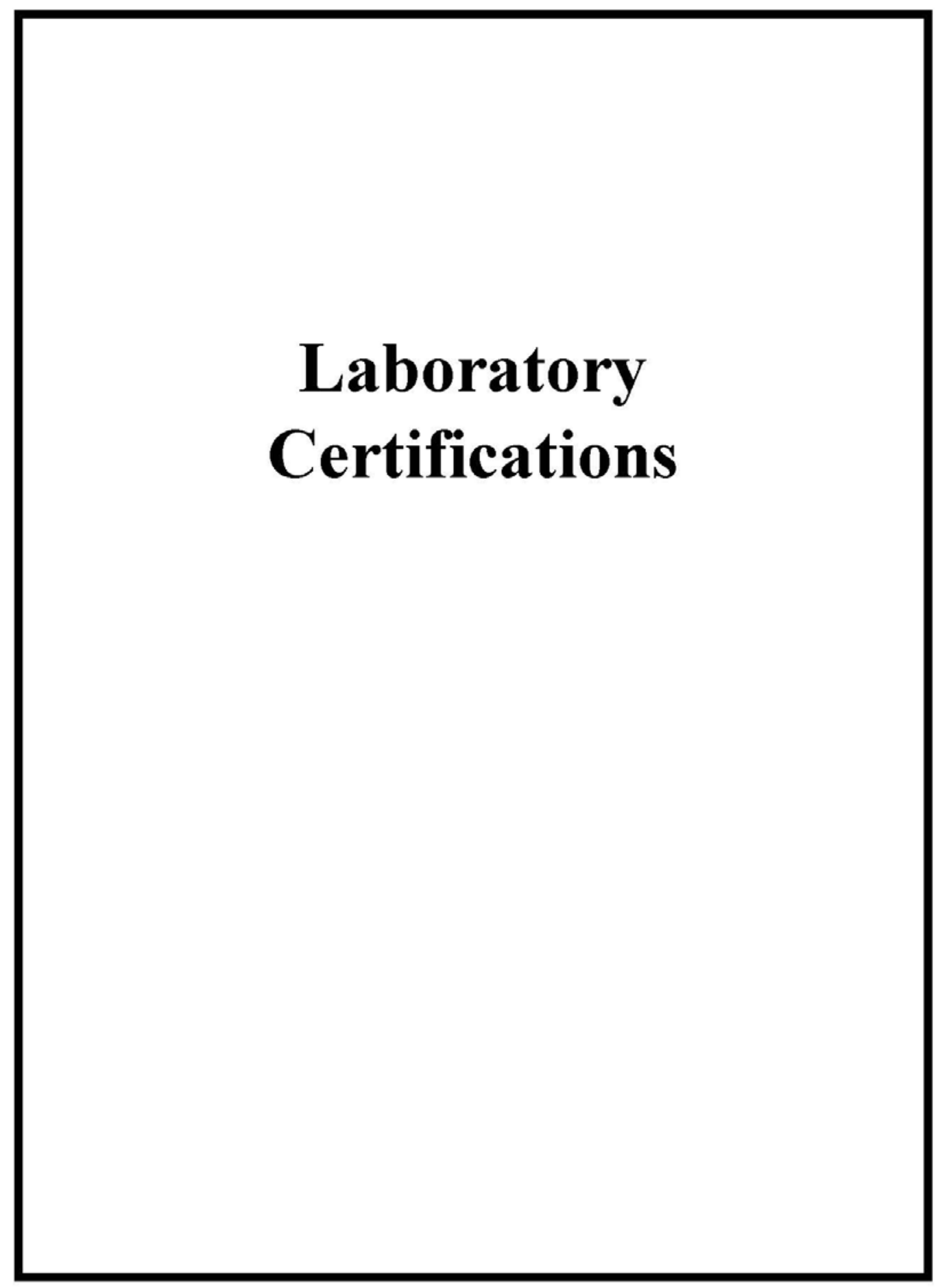

Page 10 of 94 
List of current GEL Certifications as of 30 March 2018

\begin{tabular}{|c|c|}
\hline State & Certification \\
\hline Alaska & $17-018$ \\
\hline Arkansas & $88-0651$ \\
\hline CLIA & 42D0904046 \\
\hline California & 2940 \\
\hline Colorado & $\mathrm{SC} 00012$ \\
\hline Connecticut & $\mathrm{PH}-0169$ \\
\hline Delaware & SC00012 \\
\hline DoD ELAP/ ISO17025 A2LA & 2567.01 \\
\hline Florida NELAP & E87156 \\
\hline Foreign Soils Permit & $\mathrm{P} 330-15-00283, \mathrm{P} 330-15-00253$ \\
\hline Georgia & $\mathrm{SC} 00012$ \\
\hline Georgia SDWA & 967 \\
\hline Hawaii & $\mathrm{SC} 00012$ \\
\hline Idaho Chemistry & SC00012 \\
\hline Idaho Radiochemistry & $\mathrm{SC} 00012$ \\
\hline Illinois NELAP & 200029 \\
\hline Indiana & $\mathrm{C}-\mathrm{SC}-01$ \\
\hline Kansas NELAP & E-10332 \\
\hline Kentucky SDWA & 90129 \\
\hline Kentucky Wastewater & 90129 \\
\hline Louisiana NELAP & 03046 (AI33904) \\
\hline Louisiana SDWA & LA180011 \\
\hline Maryland & 270 \\
\hline Massachusetts & $\mathrm{M}-\mathrm{SC} 012$ \\
\hline Michigan & 9976 \\
\hline Mississippi & SC00012 \\
\hline Nebraska & $\mathrm{NE}-\mathrm{OS}-26-13$ \\
\hline Nevada & SC000122018-1 \\
\hline New Hampshire NELAP & 205415 \\
\hline New Jersey NELAP & $\mathrm{SC} 002$ \\
\hline New Mexico & $\mathrm{SC} 00012$ \\
\hline New York NELAP & 11501 \\
\hline North Carolina & 233 \\
\hline North Carolina SDWA & 45709 \\
\hline North Dakota & $\mathrm{R}-158$ \\
\hline Oklahoma & 9904 \\
\hline Pennsylvania NELAP & $68-00485$ \\
\hline Puerto Rico & $\mathrm{SC} 00012$ \\
\hline S. Carolina Radiochem & 10120002 \\
\hline South Carolina Chemistry & 10120001 \\
\hline Tennessee & TN 02934 \\
\hline Texas NELAP & T104704235-18-13 \\
\hline Utah NELAP & SC000122018-26 \\
\hline Vermont & VT87156 \\
\hline Virginia NELAP & 460202 \\
\hline Washington & $\mathrm{C} 780$ \\
\hline West Virginia & 997404 \\
\hline
\end{tabular}

Page 11 of 94 


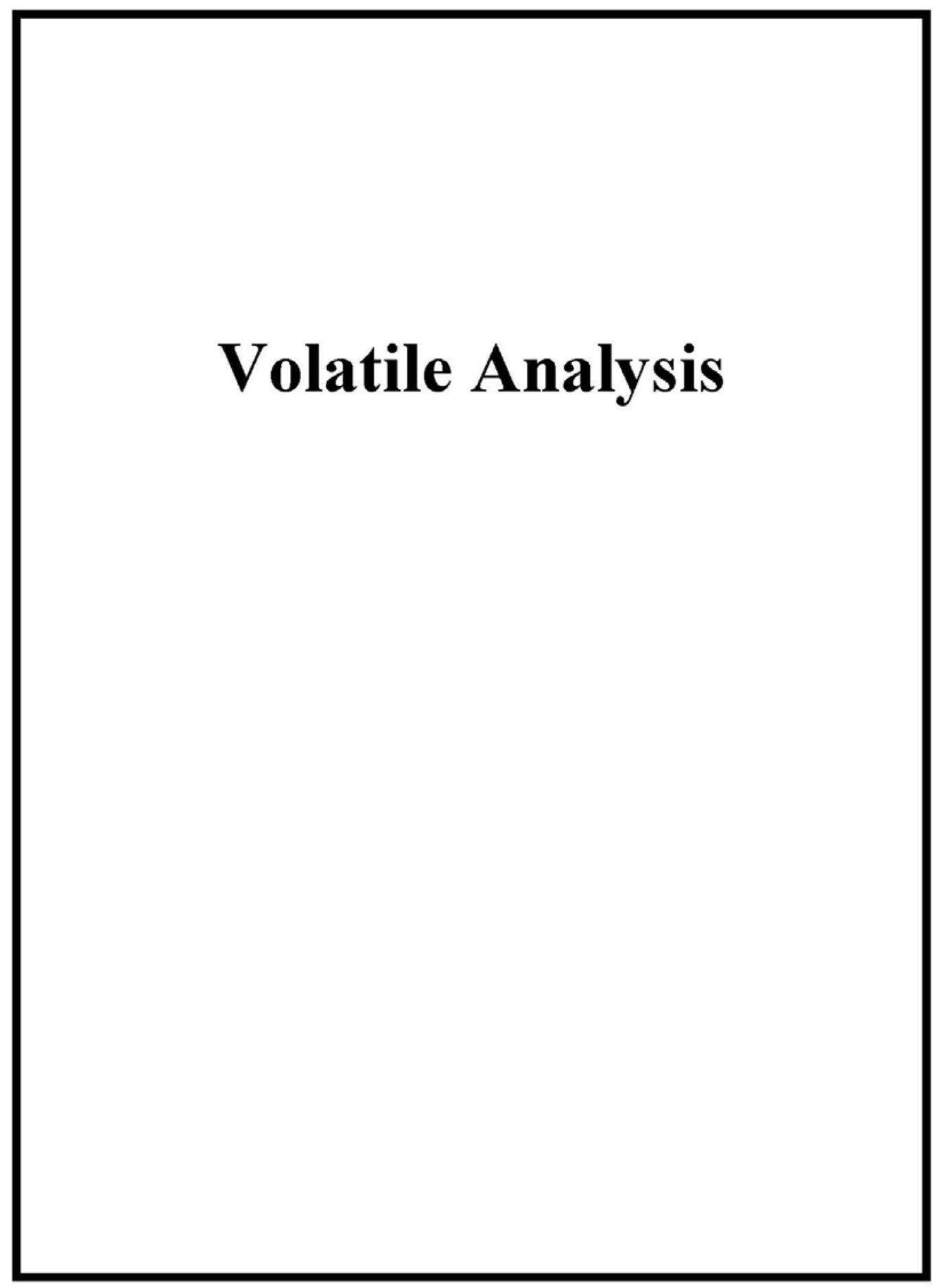

Page 12 of 94 


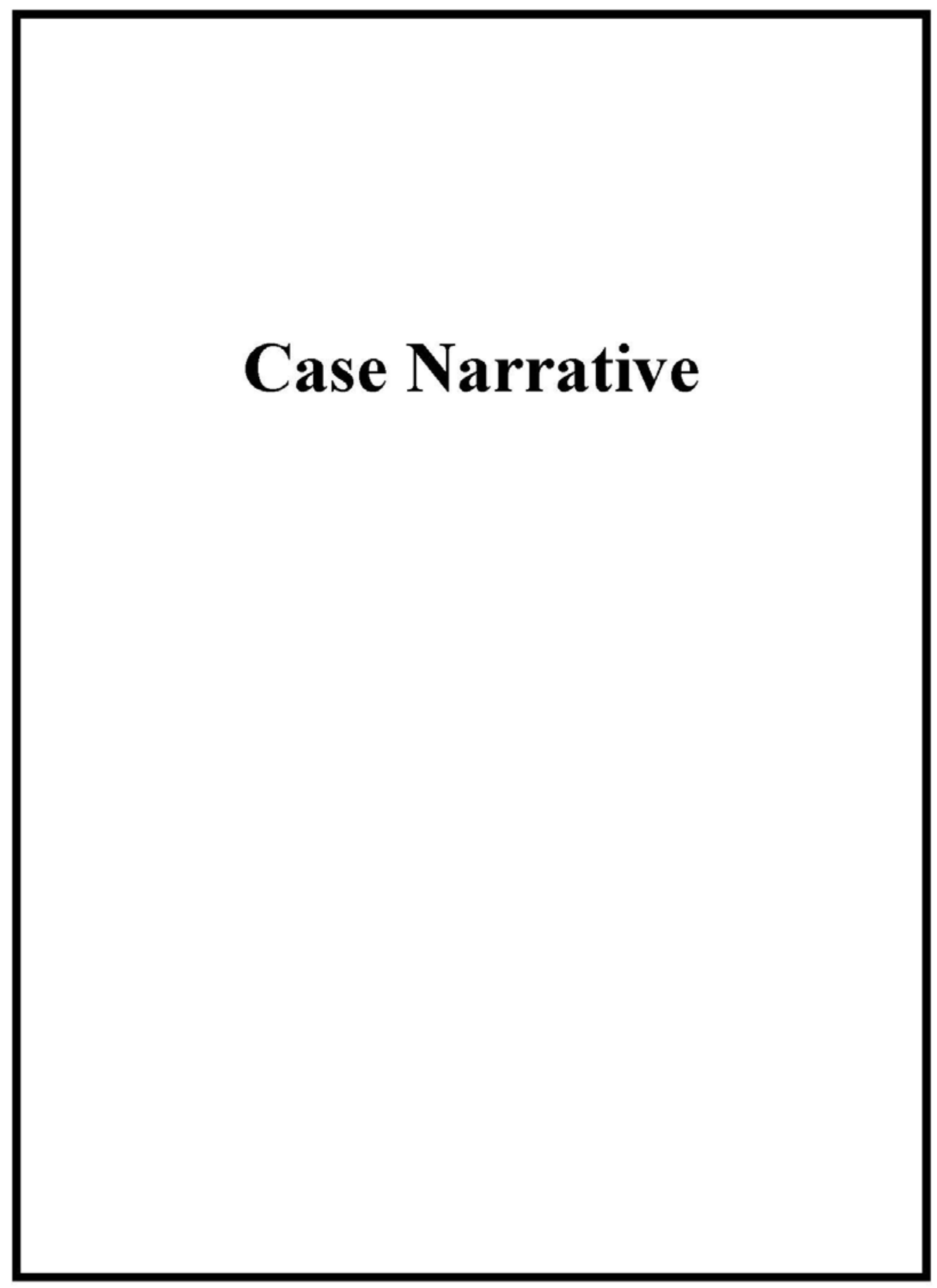

Page 13 of 94 


\section{GC/MS Volatile \\ Technical Case Narrative \\ North Wind - Portage (POEN) \\ SDG \#: $\mathbf{4 4 6 5 1 7}$}

Product: Volatile Organic Compounds (VOC) by Gas Chromatograph/Mass Spectrometer Analytical Method: SW846 8260B

Analytical Procedure: GL-OA-E-038 REV\# 26

Analytical Batch: 1751482

Preparation Method: SW846 5035

Preparation Procedure: GL-OA-E-039 REV\# 12

Preparation Batch: 1751481

The following samples were analyzed using the above methods and analytical procedure(s).

\section{GEL Sample ID\#}

446517001

446517002

446517003

446517004

1203999288

1203999289

1203999290

\author{
Client Sample Identification \\ CFA18001 - Soil Location \#1 \\ CFA18002 - Soil Location \#2 \\ CFA18003 - Soil Location \#3 \\ CFA18003 - DUP - Soil Location \#3 \\ Method Blank (MB) \\ Laboratory Control Sample (LCS) \\ Laboratory Control Sample Duplicate (LCSD)
}

The samples in this SDG were analyzed on a "dry weight" basis.

\section{Data Summary:}

There are no exceptions, anomalies or deviations from the specified methods. All sample data provided in this report met the acceptance criteria specified in the analytical methods and procedures for initial calibration, continuing calibration, instrument controls and process controls where applicable.

\section{Certification Statement}

Where the analytical method has been performed under NELAP certification, the analysis has met all of the requirements of the NELAC standard unless otherwise noted in the analytical case narrative.

Page 14 of 94 


\title{
GEL LABORA TORIES LLC
}

2040 Savage Road Charleston SC 29407 - (843)556-8171 - www gel com

\author{
Qualifier Definition Report \\ for
}

POEN00 4 N or th Wind- Portage (8-00000013)

Client SDG: 446517 GEL Work Order: 446517

\section{The Qualifiers in this report are defined as follows:}

* A qualify control anslyte re covery is outside of specified acceptance aiteria

wat Arityte is a surnogate compound

$U$ Anable was amalyzed for, but not detected above the MDL, MD A, MDC or L OD .

DL Indic ates that sample is dibited.

RA Indic ates that sample is re-analyzed withoutre-extraction.

RE Indicates that sampl is re-extracted.

\section{Review/Validation}

GEL requires all analytical data to be verf ied by a qualif ied data reviewer. In addition, all clp-like de liverables receive a third level review of the fractional data package.

The following data validator verified the information pre serted in this data report:
Signaure: 5rind to buberto
Name: Erin Haubert
D\#e: 04 APR 2018
Title: Data Validator 


\section{Sample Data Summary}

Page 16 of 94 


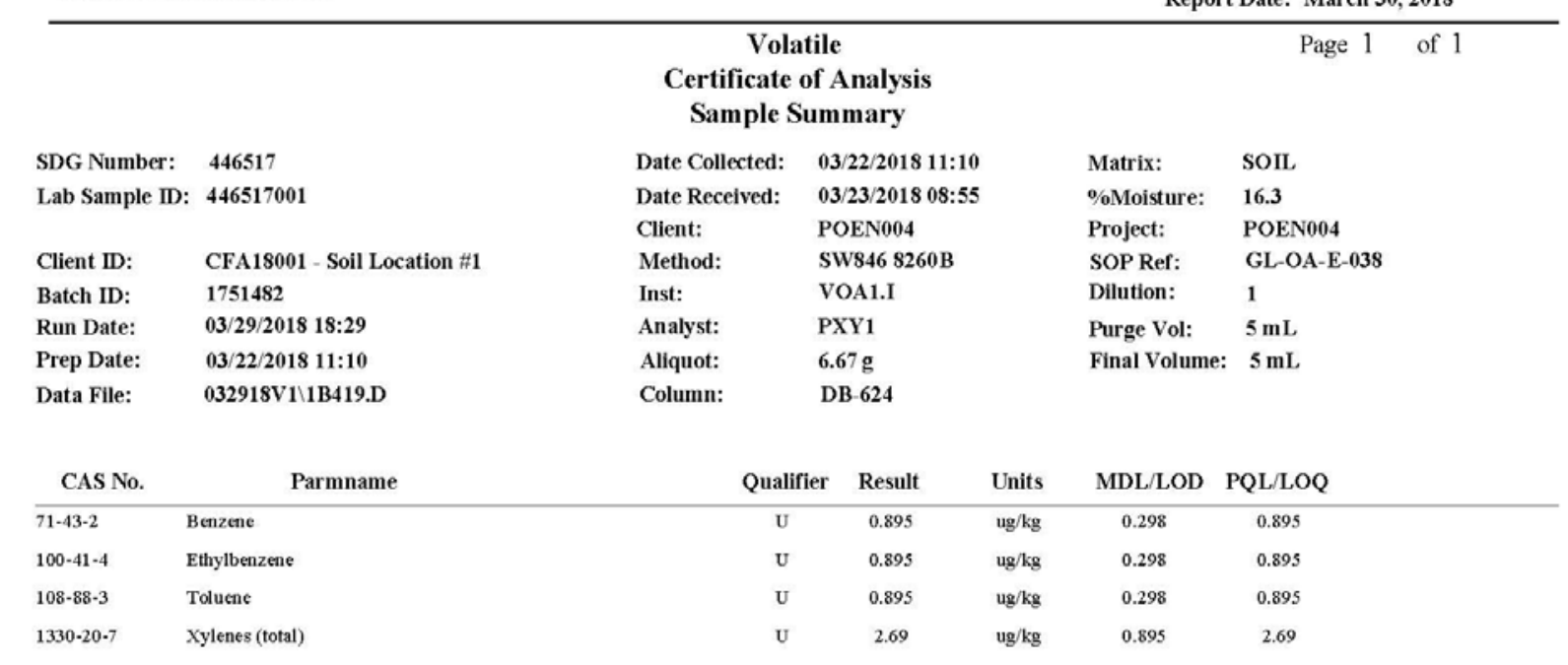

Page 17 of 94 


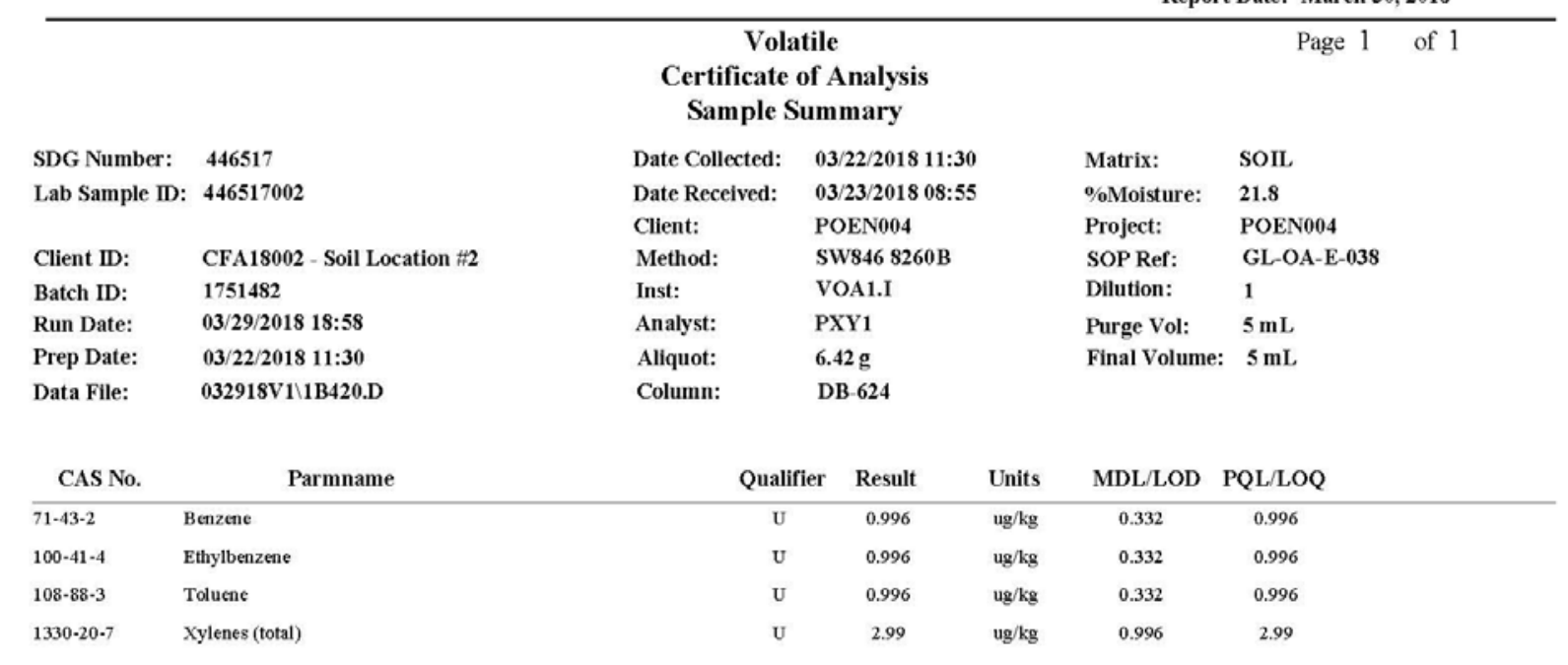

Page 18 of 94 


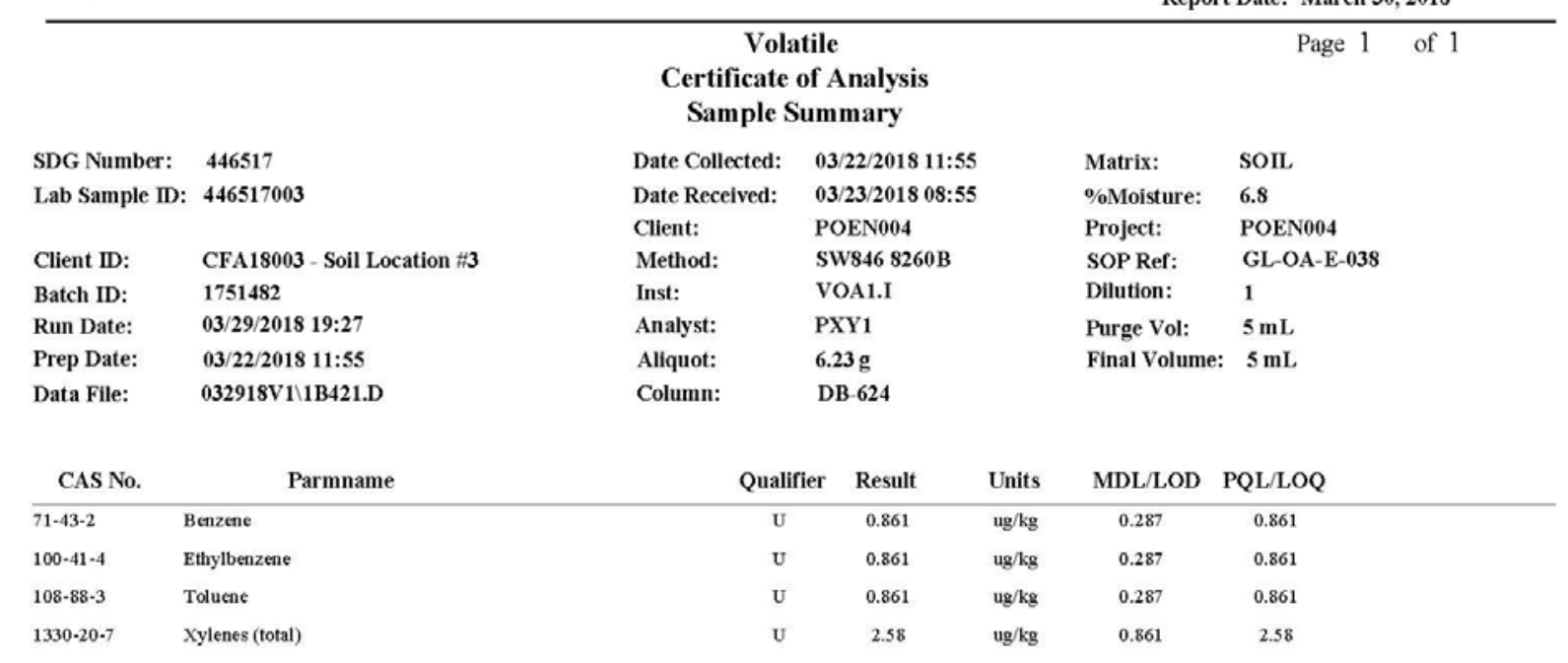

Page 19 of 94 


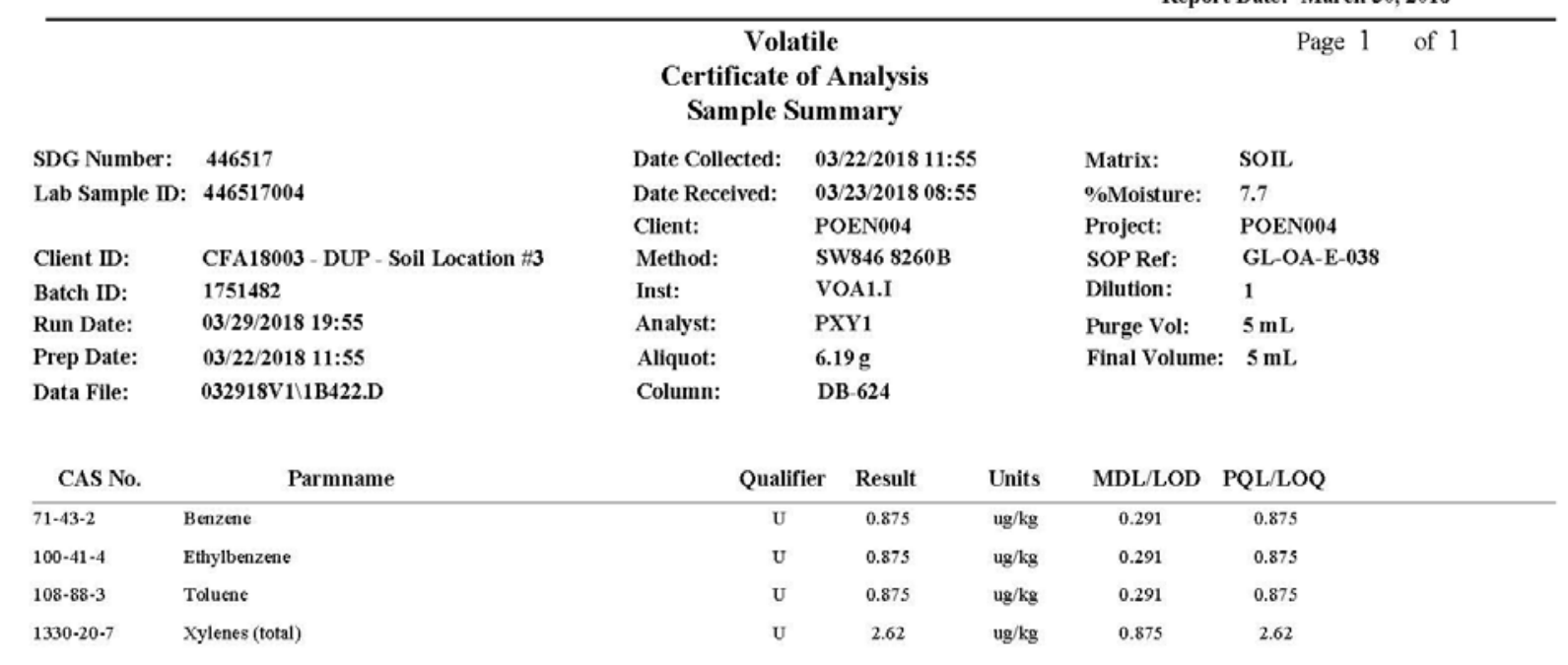

Page 20 of 94 


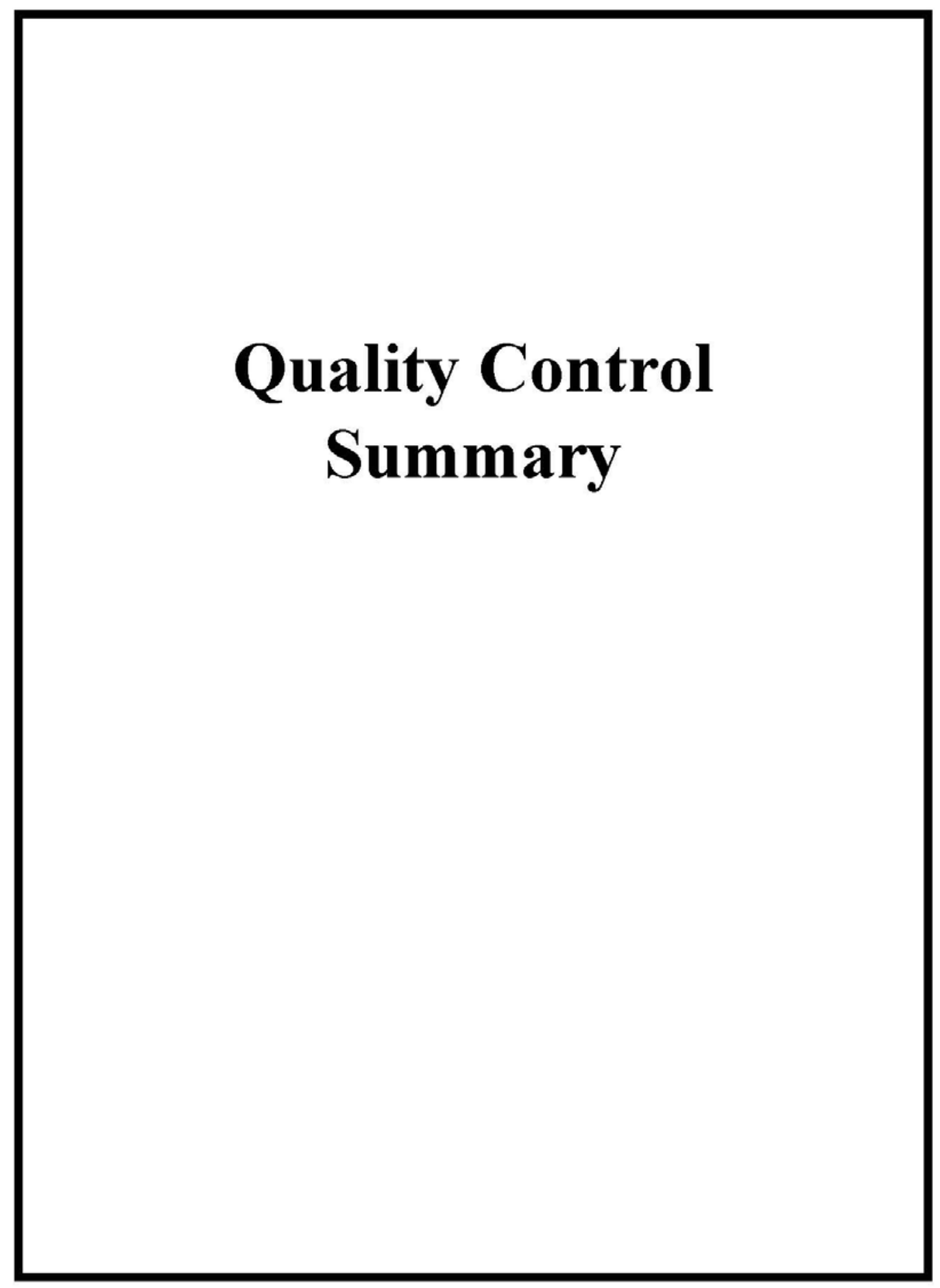

Page 21 of 94 


\section{Volatile \\ Surrogate Recovery Report}

Page 1

SDG Number: 446517

Matrix Type: SOLID

\begin{tabular}{llccc}
\hline Sample ID & Client ID & $\begin{array}{c}\text { DCED4 } \\
\text { \%REC }\end{array}$ & $\begin{array}{l}\text { TOL } \\
\text { \% REC }\end{array}$ & $\begin{array}{l}\text { BFB } \\
\text { \% REC }\end{array}$ \\
\hline 1203999289 & LCS for batch 1751481 & 94 & 93 & 100 \\
1203999290 & LCSD for batch 1751481 & 93 & 94 & 101 \\
1203999288 & MB for batch 1751481 & 93 & 93 & 101 \\
446517001 & CFA18001 - Soil Location \#1 & 89 & 94 & 103 \\
446517002 & CFA18002 - Soil Location \#2 & 91 & 93 & 101 \\
446517003 & CFA18003 - Soil Location \#3 & 94 & 93 & 103 \\
446517004 & $\quad$ CFA18003 - DUP - Soil Location \#3 & 92 & 94 & 107 \\
& & & & \\
Surrogate & & Acceptance Limits & \\
DCED4 = 1,2-Dichloroethane-d4 & $(81 \%-124 \%)$ & & \\
TOL $\quad$ Toluene-d8 & $(81 \%-120 \%)$ & & \\
BFB = Bromofluorobenzene & $(70 \%-130 \%)$ & & \\
* Recovery outside Acceptance Limits & & \\
\# Column to be used to flag recovery values & & \\
D Sample Diluted &
\end{tabular}

Page 22 of 94 


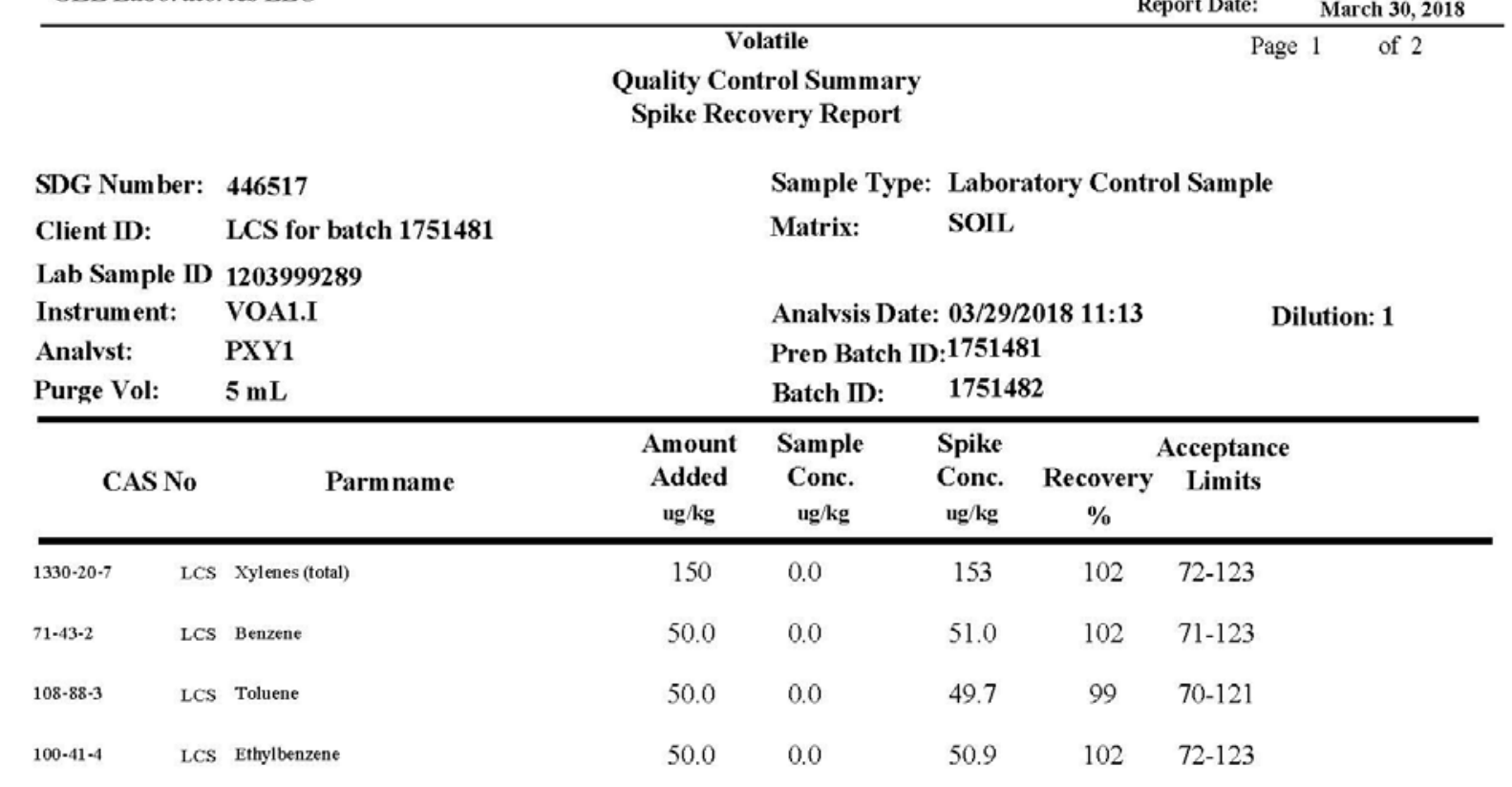




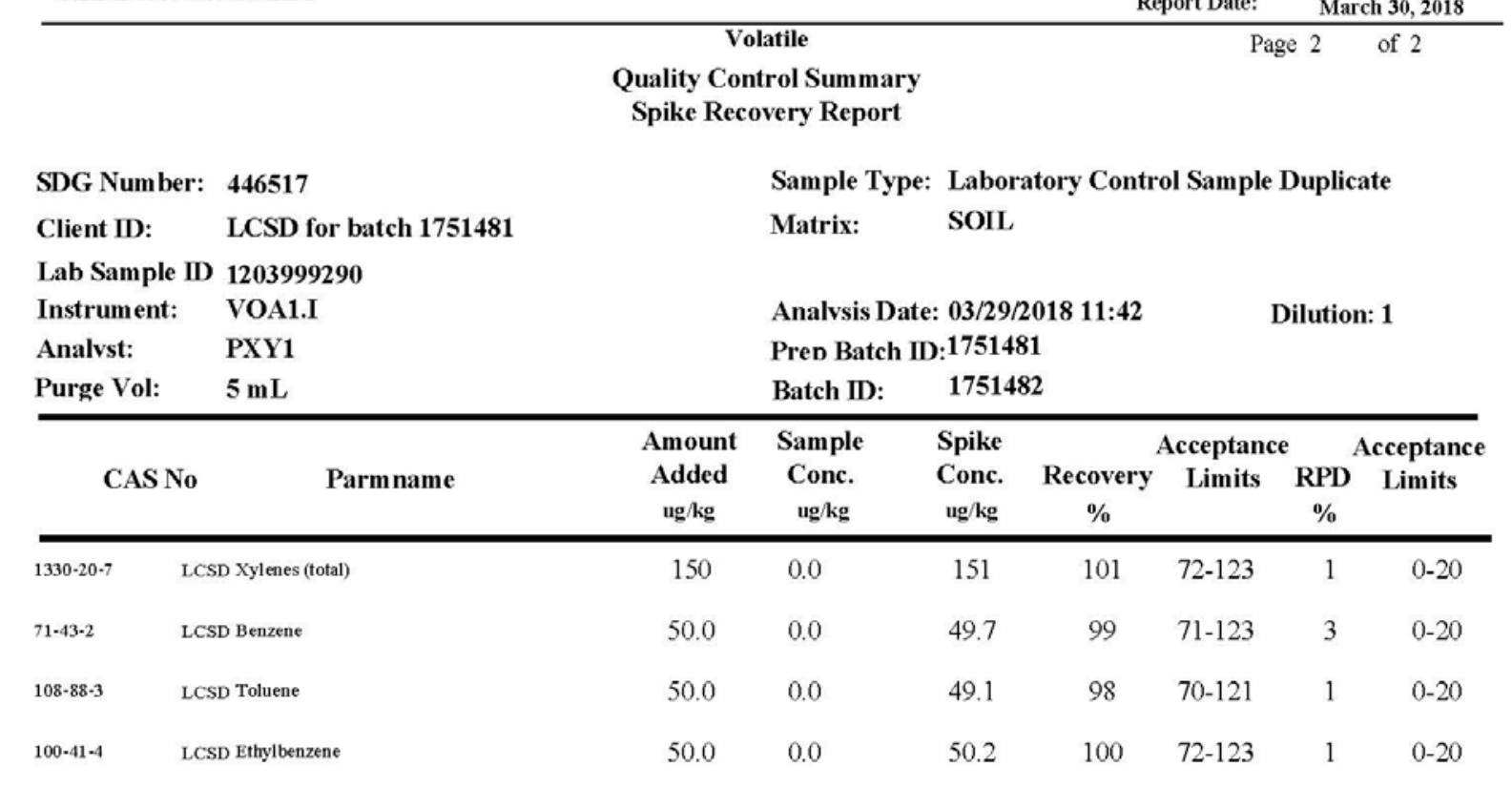




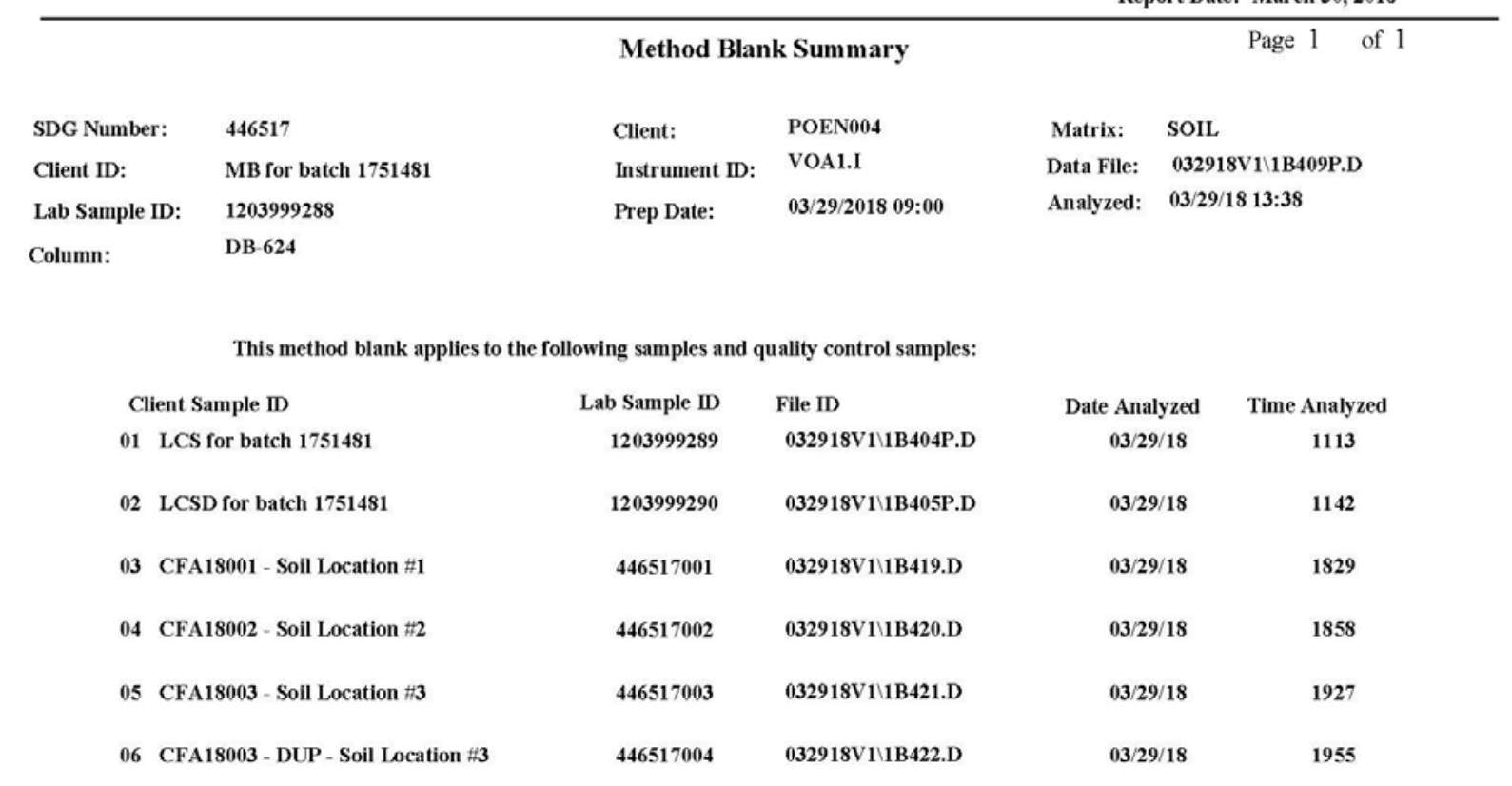


Instrument Performance Check

BROMOFLUOROBENZENE

Lab Name GEL Laboratories LLC

Instrument ID: VOAl.I

Column Description: DB-624
Client SDG: 446517

Injection Date/Time: 26-MAR-18 13:47

\begin{tabular}{|c|c|c|}
\hline $\mathbf{m} / \mathbf{e}$ & Ion Abundance Criteria & \% Relative Abundance \\
\hline 50 & $15.0-40.0 \%$ of mass 95 & 22.2 \\
\hline 75 & $30.0-60.0 \%$ of mass 95 & 50.5 \\
\hline 95 & Base Peak, $100 \%$ Relative Abundance & 100 \\
\hline 96 & $5.0-9.0 \%$ of mass 95 & 5.9 \\
\hline 173 & Less than $2.0 \%$ of mass 174 & 0 \\
\hline 174 & $50.0-100.0 \%$ of mass 95 & 67.5 \\
\hline 175 & $5.0-9.0 \%$ of mass 174 & 9 \\
\hline 176 & $95.0-101.0 \%$ of mass 174 & 98.7 \\
\hline 177 & $5.0-9.0 \%$ of mass 176 & 5.9 \\
\hline
\end{tabular}

THIS CHECK APPLIES TO THE FOLLOWING SAMPLES, MS, MSD, LCS, LCSD,BLANKS AND STANDARDS

\begin{tabular}{|c|c|c|c|}
\hline $\begin{array}{c}\text { Client } \\
\text { Sample II }\end{array}$ & $\begin{array}{c}\text { Lab } \\
\text { Sample ID }\end{array}$ & $\begin{array}{c}\text { Lab } \\
\text { File ID }\end{array}$ & $\begin{array}{c}\text { Time } \\
\text { Analyzed }\end{array}$ \\
\hline ICALMIX[A] & W1VM180326-01 & 032618V1\1B102.D & 26-MAR-18 14:10 \\
\hline ICALMIX[A] & W1 VM180326-02 & 032618V1\1B103.D & 26-MAR-18 14:39 \\
\hline ICALMIX[A] & W1VM180326-03 & 032618V1\1B104.D & 26-MAR-18 15:08 \\
\hline ICALMIX[A] & W1VM180326-04 & 032618V1\1B105.D & 26-MAR-18 15:37 \\
\hline ICALMIX[A] & W1VM180326-06 & 032618V1\1B107.D & 26-MAR-18 16:35 \\
\hline ICALMIX[A] & W1 VM180326-07 & 032618V1\1B108.D & 26-MAR-18 17:04 \\
\hline ICALMIX[A] & W1VM180326-08 & 032618V1\1B109.D & 26-MAR-18 17:32 \\
\hline ICALMIX[A] & W1VM180326-09 & 032618V1\1B110.D & 26-MAR-18 18:01 \\
\hline ICALMIX[A] & W1 VM180326-05 & 032618V1\1B112.D & 26-MAR-18 18:58 \\
\hline ICVMIX[A]01 & W1VM180326-10 & 032618V1\1B121.D & 26-MAR-18 23:15 \\
\hline
\end{tabular}


Instrument Performance Check

BROMOFLUOROBENZENE

Lab Name GEL Laboratories LLC

Instrument ID: VOAl.I

Column Description: DB-624
Client SDG: 446517

Injection Date/Time: 29-MAR-18 09:53

\begin{tabular}{|c|c|c|}
\hline $\mathbf{m} / \mathbf{e}$ & Ion Abundance Criteria & \% Relative Abundance \\
\hline 50 & $15.0-40.0 \%$ of mass 95 & 20.2 \\
\hline 75 & $30.0-60.0 \%$ of mass 95 & 50.1 \\
\hline 95 & Base Peak, $100 \%$ Relative Abundance & 100 \\
\hline 96 & $5.0-9.0 \%$ of mass 95 & 7.7 \\
\hline 173 & Less than $2.0 \%$ of mass 174 & 0 \\
\hline 174 & $50.0-100.0 \%$ of mass 95 & 8.9 \\
\hline 175 & $5.0-9.0 \%$ of mass 174 & 98.3 \\
\hline 176 & $95.0-101.0 \%$ of mass 174 & 7.2 \\
\hline 177 & $5.0-9.0 \%$ of mass 176 & \\
\hline
\end{tabular}

THIS CHECK APPLIES TO THE FOLLOWING SAMPLES, MS, MSD, LCS, LCSD,BLANKS AND STANDARDS

\begin{tabular}{|c|c|c|c|}
\hline $\begin{array}{c}\text { Client } \\
\text { Sample ID }\end{array}$ & $\begin{array}{c}\text { Lab } \\
\text { Sample ID }\end{array}$ & $\begin{array}{c}\text { Lab } \\
\text { File ID }\end{array}$ & $\begin{array}{c}\text { Time } \\
\text { Analyzed }\end{array}$ \\
\hline CCVMIX[A]01 & W1VM180329-01 & 032918V1\1B402.D & 29-MAR-18 10:16 \\
\hline BLK01LCS & 1203999289 & 032918V1\1B404P.D & 29-MAR-18 11:13 \\
\hline BLK01LCSD & 1203999290 & 032918V1\1B405P.D & 29-MAR-18 11:42 \\
\hline BLK01 & 1203999288 & 032918V1\1B409P.D & 29-MAR-18 13:38 \\
\hline CFA18001 - Soil Location \#1 & 446517001 & 032918V1\1B419.D & 29-MAR-18 18:29 \\
\hline CFA18002 - Soil Location \#2 & 446517002 & 032918V1\1B420.D & 29-MAR-18 18:58 \\
\hline CFA18003 - Soil Location \#3 & 446517003 & 032918V1\1B421.D & 29-MAR-18 19:27 \\
\hline CFA18003 - DUP - Soil Location & 446517004 & 032918V1\1B422.D & 29-MAR-18 19:55 \\
\hline
\end{tabular}

Page 27 of 94 


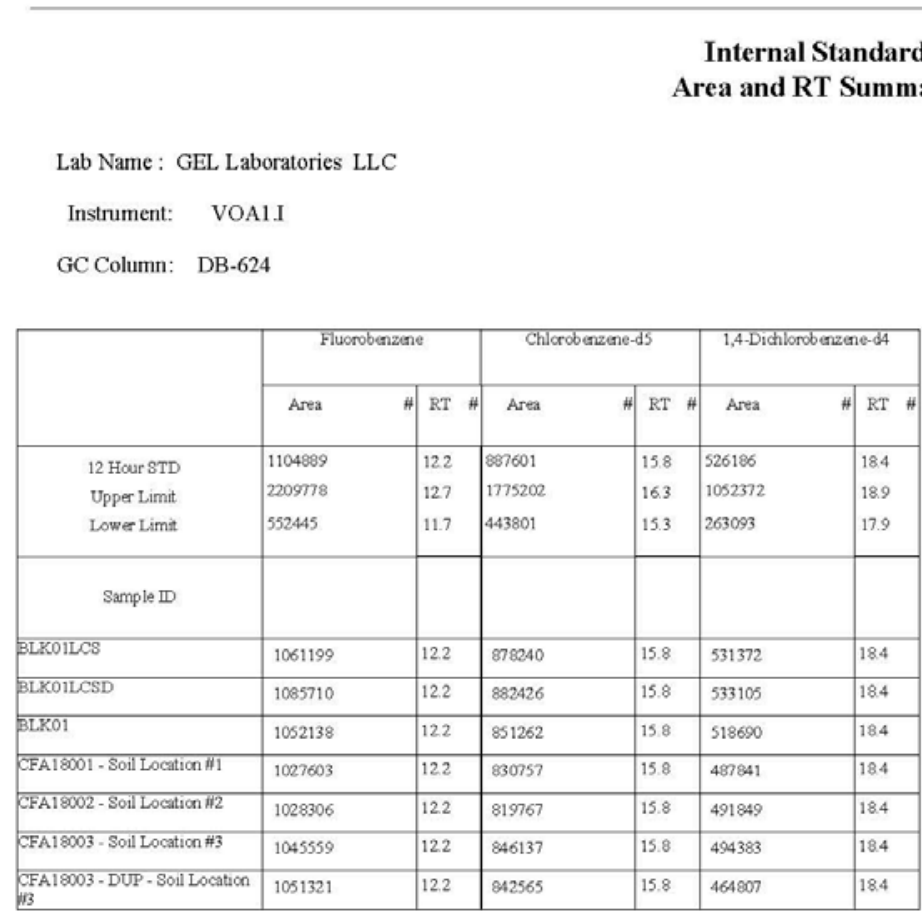

Page 1 of 1

\title{
Area and RT Summary
}

Client SDG: $\quad 446517$

STD Analysis Time: $\quad$ 29-MAR-18 10:16

Data File: 032918 V1 1 B402.D

\begin{abstract}
Area Upper Limit $=+100 \%$ of internal standard area
Area Lower Limit $=-50 \%$ of internal standard area

RT Upper Limit $=+0.50$ minutes of internal standard RT

RT Lower Limit $=-0.50$ minutes of internal standard RT

\# Column used to flag values outside QC limits with an asterisk

"Value outside of QC Limits
\end{abstract}

Page 28 of 94 


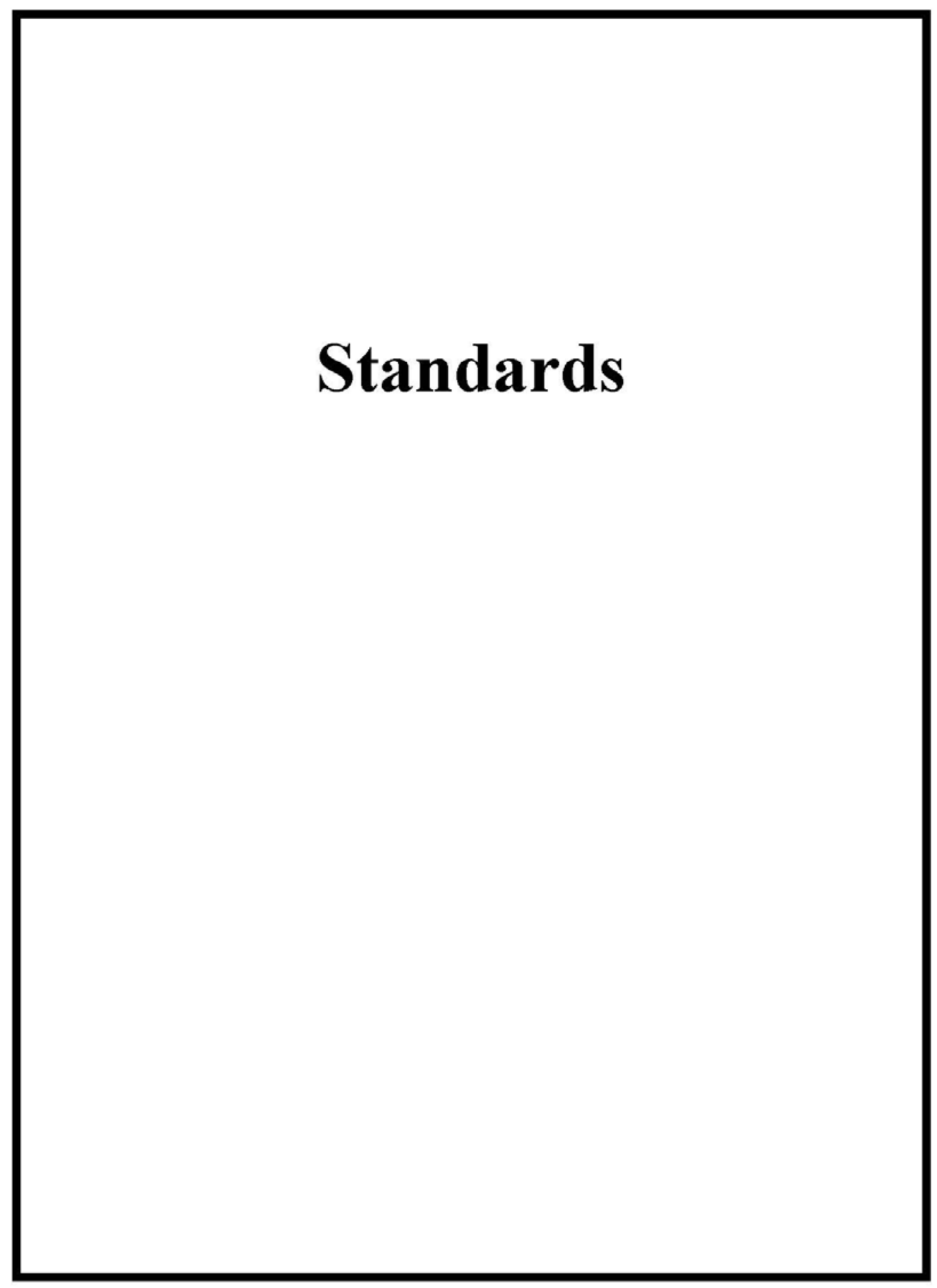

Page 29 of 94 


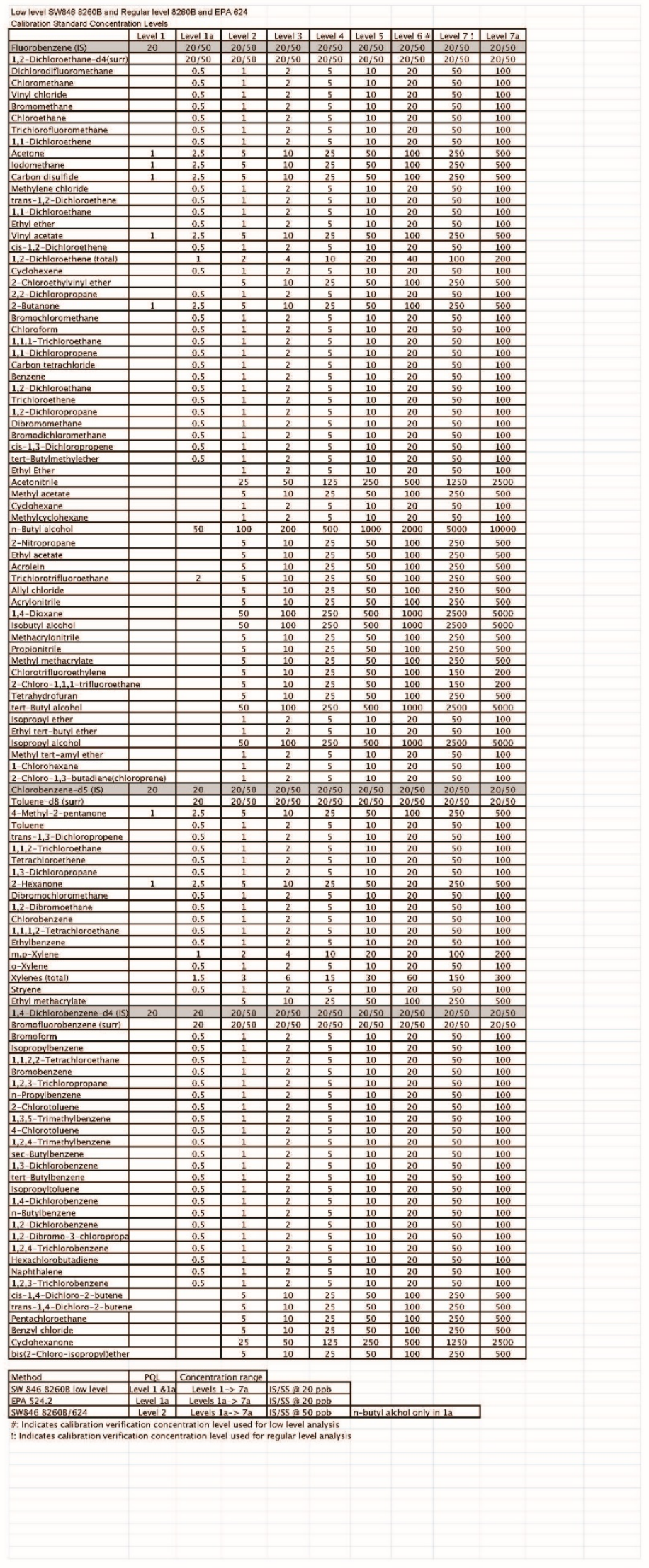

Page 30 of 94 
Calibration History Report VOAl

GEL Laboratories, LLC

Method File : C: \msdchem $\backslash 1 \backslash$ DATA $\backslash 032618 V 1 \backslash$ VOA1-8260-032618.M

Integrator : (RTE Integrator) Response via : Initial Calibration

Cal Lvl:8 Amt:0.00 Last Updated with: C: \msdchem \1 DATA $\backslash 032618$ V1 $\backslash 1 \mathrm{~B} 102 . \mathrm{D}$

| Injection Date |Mix| Calibration File
|26 Mar 2018 14:10 |A |C: 1 msdchem $\backslash$ 1 $\backslash$ DATA $\backslash 032618 V 1 \backslash 1 B 102 . \mathrm{D}$

Cal Lvl:1 Amt:1.00 Last Updated with: C: \msdchem $\backslash 1 \backslash \mathrm{DATA} \backslash 032618 \mathrm{~V} 1 \backslash 1 \mathrm{~B} 114 . \mathrm{D}$

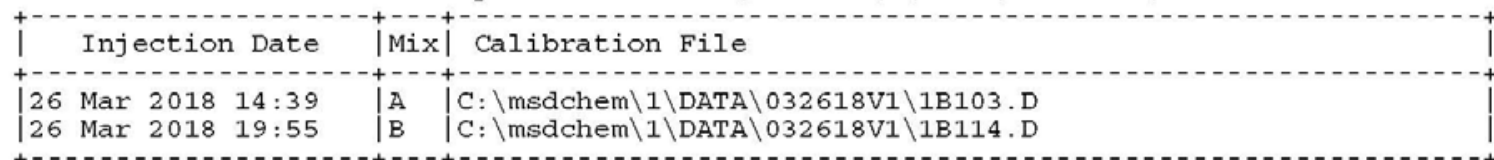

Cal Lvl:2 Amt:2.00 Last Updated with: C: \msdchem $\backslash 1 \backslash \mathrm{DATA} \backslash 032618 V 1 \backslash 1 \mathrm{~B} 115 . \mathrm{D}$

IMixl Calibration File

Mix Calibration File

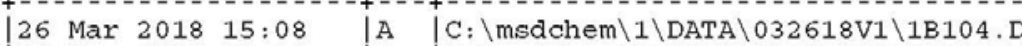

$26 \operatorname{Mar} 2018 \quad 20: 24 \quad|B| C: \backslash m s d c h e m \backslash 1 \backslash D A T A \backslash 032618 V 1 \backslash 1 B 115 . D$

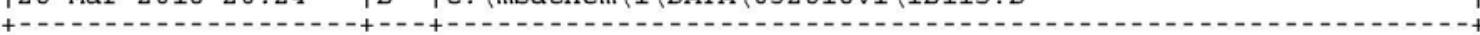

Cal Lvl:3 Amt:5.00 Last Updated with: C: \msdchem $1 \backslash$ DATA $\backslash 032618 V 1 \backslash 1 B 116 . D$

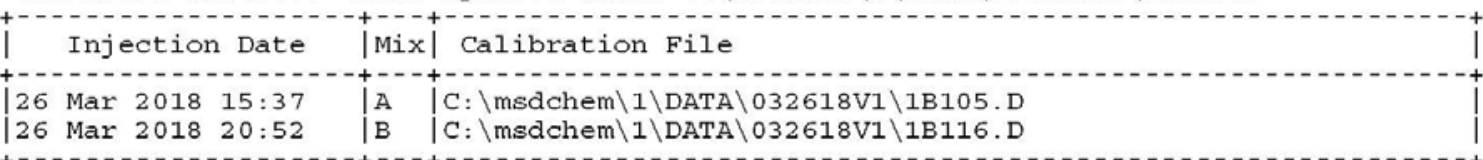

Cal Lvl:4 Amt:10.00 Last Updated with: C: \msdchem $\backslash 1 \backslash$ DATA $\backslash 032618 V 1 \backslash 1 B 117 . D$

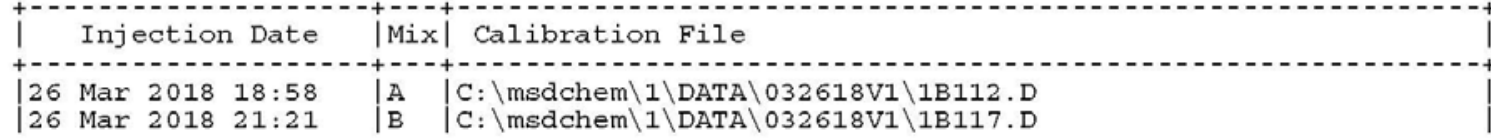

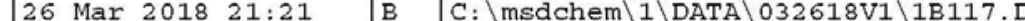

Cal Lvl:5 Amt:20.00 Last Updated with: C:\msdchem $1 \backslash$ DATA $\backslash 032618 V 1 \backslash 1 B 118 . D$

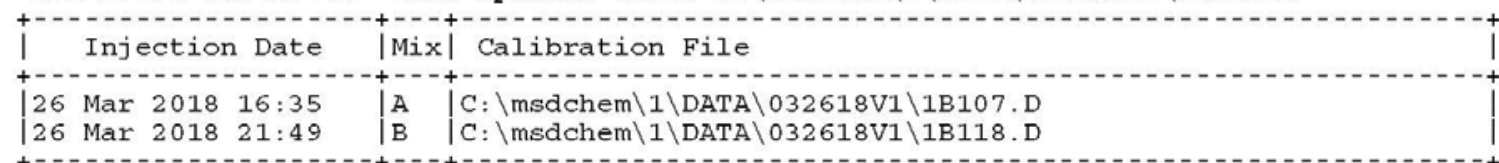

Cal Lvl:6 Amt:50.00 Last Updated with: C: msdchem $\backslash 1 \backslash$ DATA $\backslash 032618 V 1 \backslash 1 B 119 . D$

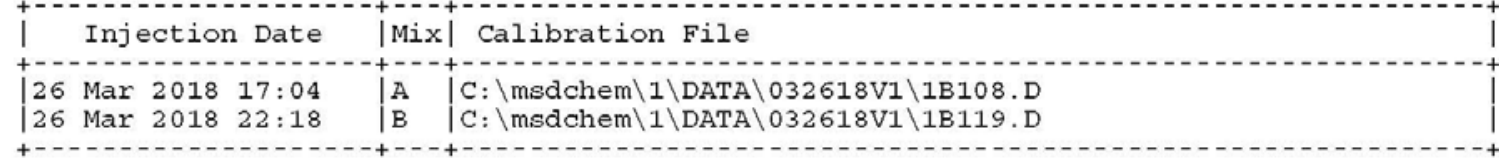

Cal Lv1:7 Amt:100.00 Last Updated with: C: \msdchem $\backslash 1 \backslash$ DATA $\backslash 032618 V 1 \backslash 1 B 120 . D$

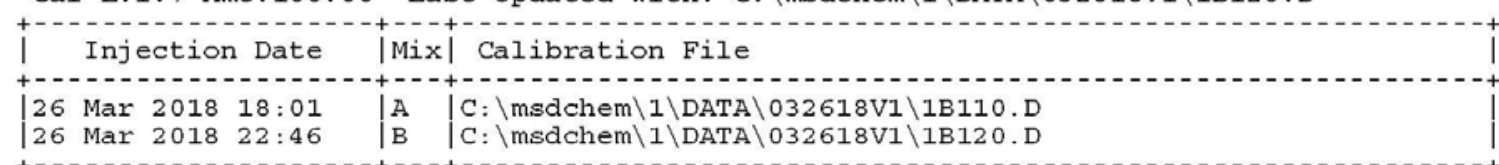

Cal Lvl:9 Amt:80.00 Last Updated with: C: \msdchem $\backslash 1 \backslash$ DATA $\backslash 032618 V 1 \backslash 1 B 109 . D$

| Injection Date $\mid$ Mix| Calibration Fil

VOA1-8260-032618.M Tue Mar $2701: 19: 042018$

Page: 1

Page 31 of 94 
Calibration History Report VOAl

GEL Laboratories, LLC

Method File : C: \msdchem \1\DATA $032618 V 1 \backslash$ VOA1-8260-032618.M

Last Update : Mon Mar 26 23:38:56 2018

Integrator : (RTE Integrator) Response via : Initial Calibration

196

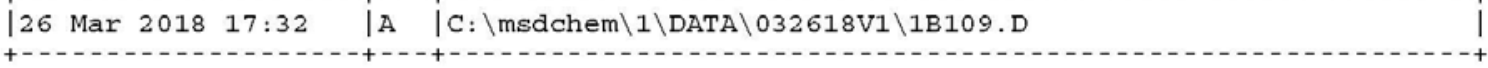

VOA1-8260-032618. M Tue Mar $2701: 19: 362018$

Page 32 of 94 
Method File : C: $\backslash$ msdchem $\backslash 1 \backslash$ DATA $\backslash 032618 V 1 \backslash$ VOA1-8260-032618.M

Method File : C: $\backslash$ msdchem $\backslash 1 \backslash$ DATA $\backslash 032618$
Last Update : Mon Mar 26 23:38:56 2018

Integrator : (RTE Integrator)

Response via : Initial Calibration

For Linear Calibration: $x=$ concentration ratio, $y=$ response ratio. $y=b+m 1(x)+m 2(x E 2)$

Cel

$03 / 27 / 2018$

\begin{tabular}{|c|c|c|c|c|c|c|c|c|c|c|}
\hline | $\mathrm{m}_{1}^{\text {Compound }} \mathrm{m} 2$ & $\begin{array}{l}8 \\
6\end{array}$ & $\begin{array}{l}1 \\
7\end{array}$ & $\begin{array}{l}2 \\
9\end{array}$ & 3 & 4 & 5 & Avg & |Curve & Exp & : $\mathrm{RSD} / \mathrm{r} 2$ \\
\hline 2) MA Dichlorodifluorometh & $0.2325581 \mid$ & $\begin{array}{l}0.2088924 \\
0.2167259\end{array}$ & $\begin{array}{l}0.2483649 \\
0.2272198\end{array}$ & 0.2603698 & 0.2346314 & $|0.2505387|$ & 0.2349 & AVRG & & 7.4532 \\
\hline 3) MPA Chloromethane & & 0.2341336 & 2296776 & 0.2354239 & 0.2436657 & 0.2275842 & i & $-\cdots$ & & \\
\hline 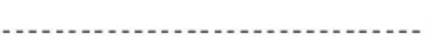 & 2156781 & $\mid 0.2070542$ & .2147298 & & & & 0.2260 & $\mid \begin{array}{l}\text { AVRG } \\
--.--\end{array}$ & & 5.4850 \\
\hline 4) MCA Vinyl chloride & 0.2274747 & 0.2259133 & 0.2361975 & 0.2349843 & 0.2263741 & 0.2281090 & 0.2252 & AVRG & & 4.2742 \\
\hline 5) MA Bromomethane & .1583241 & \begin{tabular}{|l|l|}
0.1572979 \\
0.1560454
\end{tabular} & 0.1241219 & 0.1669055 & 0.1955067 & 0.1790461 & 0.1653 & AVRG & & 13.2940 \\
\hline 6) MA Chloroethane & 1951065 & $\mid \begin{array}{l}0 \\
0.1954015 \\
0.1793082\end{array}$ & $\mid \begin{array}{l}0 \\
0.1980714 \\
0.1818580\end{array}$ & 0.1977998 & 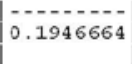 & $|0.1928280|$ & 0.1919 & |AVRG & & 3.7547 \\
\hline 7) MA Trichlorofluoromethane & 4523642 & 0.4901719 & 0.4830355 & 0.4725668 & 0.4614184 & 0.4526316 & 0.4557 & AVRG & & 6.0 \\
\hline $\begin{array}{l}\text { 8) MA Ethyl ether } \\
0.0027 \text { Y } 0.2469 \mid 0.00\end{array}$ & 263449 & $\begin{array}{r}9900 \\
511393\end{array}$ & $\begin{array}{r}10641 \\
406654\end{array}$ & 24327 & 52050 & 104561 & & $\begin{array}{l}-1 / x \\
\text { LINR }\end{array}$ & & \\
\hline 9) $\mathrm{MA}$ Acetone & 705 & $\mid \begin{array}{l}0.2001692 \\
0.1485234\end{array}$ & $\begin{array}{l}-\overline{9} \\
94\end{array}$ & 0.1724045 & 0.1713927 & 0.1496458 & - & & & $\cdots+-$ \\
\hline 10) MCA 1,1-Dichloroethylene & 0.4143312 & $\begin{array}{l}0.4043906 \\
0.3970129\end{array}$ & $\begin{array}{l}0.4155089 \\
0.4044306\end{array}$ & 0.4167753 & 0.4244260 & 0.4215178 & 0.4123 & $-\cdots$ & & 2.2912 \\
\hline 11) MA Iodomethane & 4952894 & $\begin{array}{l}0.5354416 \\
0.4440563\end{array}$ & .5446771 & 0.5400810 & 0.5461744 & 0.5283108 & 0.5121 & $-\cdots$ & & - \\
\hline 12) MA Acetonitrile & 0343681 & $\mid \begin{array}{l}0.0355543 \\
0.0324327\end{array}$ & 0.0363771 & 0.0352623 & 0.0346553 & 0.0340871 & 0.0343 & Heves & & 4.3639 \\
\hline 13) MA Methyl acetate & .0508425 & $\mid \begin{array}{l}0.0446024 \\
0.0499693\end{array}$ & $\begin{array}{l}0.0491155 \\
0.0484456\end{array}$ & 0.0503412 & 0.0516941 & 0.0515496 & 0.0496 & $-\cdots$ & & 4.6350 \\
\hline 14) MA Carbon disulfide & 7985348 & $\begin{array}{l}0.9492614 \\
0.6814759\end{array}$ & $\begin{array}{l}-1.78 \\
0.9441018 \\
0.7174578\end{array}$ & 0.9212366 & 0.9017682 & 0.8653331 & 0.8474 & AVRG & & 12.2362 \\
\hline $\begin{array}{l}\text { 15) MA Methylene chloride } \\
0.0056|0.3229| 0.00\end{array}$ & 340898 & $\begin{array}{r}11875 \\
664835\end{array}$ & $\begin{array}{r}18805 \\
530308\end{array}$ & 39688 & 75341 & 143203 & & $\begin{array}{l}-\cdots- \\
1 / x \\
\text { LINR }\end{array}$ & & 0.9995 \\
\hline $\begin{array}{l}\text { 16) MA tert-Butyl methyl eth } \\
-0.0079|0.6591| 0.00\end{array}$ & 647265 & $\begin{array}{r}9248 \\
1415395\end{array}$ & $\begin{array}{r}19310 \\
1070734\end{array}$ & 49366 & 119802 & 235839 & & \begin{tabular}{|l|l}
$1 / x$ \\
$\mid$ LINR
\end{tabular} & & 0.9972 \\
\hline
\end{tabular}

VOA1-8260-032618.M Tue Mar 27 01:20:22 2018

Page: 1 
Response Factor Report VOA1

GEL Laboratories, LLC

Method File : C: \msdchem \1\DATA $\backslash 032618 \mathrm{~V} 1 \backslash$ VOA1-8260-032618.M

Last Update: Mon Mar 26 23:38:56 2018

Integrator: (RTE Integrator)

Response via : Initial Calibration

For Linear Calibration: $x=$ concentration ratio, $y=$ response ratio. $y=b+m 1(x)+m 2(x E 2)$

\begin{tabular}{|c|c|c|c|c|c|c|c|c|c|c|c|}
\hline $\mathrm{b}$ & Compound & $\begin{array}{l}8 \\
6\end{array}$ & $\frac{1}{7}$ & $\begin{array}{l}2 \\
9\end{array}$ & 3 & 4 & 5 & Avg & |Curve & Exp & / $8 \mathrm{RSD} / \mathrm{r} 2$ \\
\hline 17) $M A$ & trans-1,2-Dichloroethyle & 283 & $\begin{array}{l}0.3898358 \\
0.3788480\end{array}$ & 0.4240649 & 0.4182697 & 0.4239597 & 0.4147848 & & JAVR & & \\
\hline & & & & .5020402 & 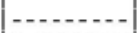 & & & & aviso & & \\
\hline 18) $M A$ & Hexane & 0.3262771 & 0.3249190 & 0.3358023 & $|0.3180659|$ & 0.3535451 & 0.3569891 & 0.3359 & AVRG & & 4.7765 \\
\hline 19) MA & Vinyl acetate & 0.5059411 & $\begin{array}{l}0.3608133 \\
0.4309509\end{array}$ & 0.4081406 & $\mid$ & 0.4850428 & 0.4912750 & 0.4503 & AVRG & & 195 \\
\hline-- & - & - $1000-1$ & $-\ldots$ & - & - & 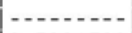 & & & $-\cdots$ & & \\
\hline 20) MPA & 1,1-Dichlo & 0.5173020 & $\begin{array}{l}0.4966998 \\
0.4911449\end{array}$ & $\begin{array}{l}0.5086306 \\
0.4961369\end{array}$ & $|0.5111773|$ & 0.5201709 & 0.5245081 & 0.5082 & AVRG & & 2.4325 \\
\hline 21) $\mathrm{MA}$ & 2-Butanone & & 0.1915718 & 0.1957543 & $\mid$ & 0.2031121 & 0.1825786 & & & & \\
\hline & & 0.1793316 & 0.1573201 & 0.1584933 & & & & 0.1833 & AVRG & & 9.5571 \\
\hline 22) MA & cis-1,2-Dichloroethy & & 0.3028457 & 0.3263865 & $|0.3288100|$ & 0.3389735 & 0.3332449 & & & & \\
\hline & & $|0.3298769|$ & 0.3056791 & 0.3110140 & & & & 0.3221 & AVRG & & 4.2245 \\
\hline 23) $\mathrm{MA}$ & 2,2-Dichloropropane & 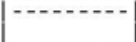 & 0.2495104 & 0.2620593 & 0.2710456 & 0.2938065 & 0.3055425 & $-\cdots$ & $\mid-\cdots$ & & $\cdots$ \\
\hline & & 0.3320564 & 0.3319159 & 0.3320443 & & & & 0.2972 & AVRG & & 11.3079 \\
\hline 24) $M A$ & Bromochloromethane & (n) & 0.1773652 & 0.1857586 & $\mid$ & 0.1797257 & 0.1795078 & & & & \\
\hline & & 0.1781791 & 0.1729483 & 0.1714010 & & & & 0.1775 & AVRG & & 2.5160 \\
\hline 25) MCA & Chloroform & & $0 .-10$. & -- & 0.5048547 & 0.6162850 & 0.5860000 & & $\mid-\cdots$ & & \\
\hline$2 0 \longdiv { 1 0 0 }$ & & .5786182 & 0.551 & 0.55 & & & & 0.5880 & AVRG & & 2906 \\
\hline & - & 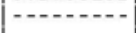 & - & -5 & & t & & $-\cdots$ & $-\cdots$ & | - - & \\
\hline 26) $M A$ & 1,1, & 0.4545101 & $\begin{array}{l}0.3918667 \\
0.4451876\end{array}$ & $\mid \begin{array}{l}0.3897199 \\
0.4446420\end{array}$ & 0.404 & 0.43 & 0.44 & 0.4260 & AVRG & & 6.1771 \\
\hline 27) MA & Cyclohexane & & 0.3683663 & 0.406 & $0.4285646 \mid$ & 0.4390510 & 0.4432647 & & & & \\
\hline & & 4352720 & 0.4017 & 61 & & & & 0.4168 & AVRG & & 004 \\
\hline 28) MA & 1,1-Dichloropropene & & 0.3368874 & $\overline{66}$ & 0.3626150 & 0,3766119 & 0.3743477 & & & & \\
\hline & & 0.3724604 & 0.347 & 67 & & & & 0.3601 & AVRG & & 042 \\
\hline & -1 & & & & & & & & & -- & \\
\hline 29) MA & Carbon tetrachloride & $|0.4387564|$ & $\begin{array}{l}0.4071468 \\
0.4137434\end{array}$ & $\begin{array}{l}0.4165027 \\
0.4208563\end{array}$ & $\mid 0.4249995$ & 0.4340238 & 0.4414642 & 0.4247 & AVRG & & 2.9191 \\
\hline & & & & & D 1605005 & & & & & & \\
\hline 30) $\mathrm{SA}$ & 1,2-Dichloroet & 650263 & $\begin{array}{l}0.1657030 \\
0.1650191\end{array}$ & $\begin{array}{l}0.1655181 \\
0.1616716\end{array}$ & 0.1605205 & 0.1631044 & 0.1619280 & 0.1636 & AVRG & & 1.2311 \\
\hline 31) $M A$ & 1,2-Dichloroe & & 0.47 & 46 & $\left|\begin{array}{l}0 .-1 .-1 \\
0.4686598\end{array}\right|$ & 0.4764902 & 0.4652375 & & & & \\
\hline & & 0.4554705 & 0.4258460 & 0.4274934 & & & & 0.4594 & AVRG & & 4.725 \\
\hline
\end{tabular}

VOA1-8260-032618.M Tue Mar 27 01:20:22 2018

Page: 2 
Response Factor Report VOA1

GE Iaboratories, LLC

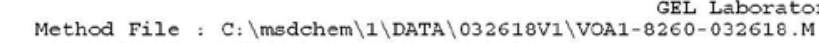

Last Update : Mon Mar $26 \quad 23: 38: 562018$

Integrator: (RTE Integrator)

Response via : Initial Calibration

For Linear Calibration: $x=$ concentration ratio, $y=$ response ratio. $y=b+m 1(x)+m 2(x E 2)$

\begin{tabular}{|c|c|c|c|c|c|c|c|c|c|c|}
\hline $\mathrm{b} \quad \mid \begin{array}{c}\text { Compound } \\
\mathrm{m} 1\end{array}$ & $\begin{array}{l}8 \\
6\end{array}$ & $\begin{array}{l}1 \\
7\end{array}$ & $\begin{array}{l}2 \\
9\end{array}$ & 3 & 4 & 5 & Avg & |Curve & Exp & : $\mathrm{RSD} / \mathrm{r} 2$ \\
\hline 32) MA Ben & $|1.0886789|$ & $\left|\begin{array}{l}1.1482798 \\
0.9958207\end{array}\right|$ & $\left|\begin{array}{l}1.1691111 \\
1.0085417\end{array}\right|$ & 1.1579361 & 1.1604116 & 1.1270888 & 1.1070 & AVRG & & 6.2775 \\
\hline 33) MA Cyclohexene & 0.4803221 & $\left|\begin{array}{l}0.4103866 \\
0.4462752\end{array}\right|$ & $\left|\begin{array}{l}0.0-1 \\
0.4395528 \\
0.4580332\end{array}\right|$ & 0.4560989 & 0.4879435 & 0.4787984 & 0.4572 & AVRG & & 5.5892 \\
\hline $\begin{array}{l}\text { 34) MA n-Butyl alcohol } \\
-0.0055|0.0084| 0.00\end{array}$ & $\begin{array}{r}5910 \\
892002\end{array}$ & $\left|\begin{array}{r}11283 \\
1723839\end{array}\right|$ & $\begin{array}{r}27734 \\
1357268\end{array}$ & 74080 & 166764 & 353927 & & $\begin{array}{l}--\cdot- \\
1 / x \\
\text { LINR }\end{array}$ & & 0.9984 \\
\hline 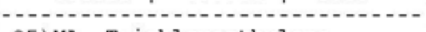 & & $-\cdots-1$ & (n) & 3 & ดอง & 1 & & & & \\
\hline 35) MA Trichloroe & .2992549 & $\left|\begin{array}{l}0.2864051 \\
0.2849576\end{array}\right|$ & $\left|\begin{array}{l}0.3113832 \\
0.2847453\end{array}\right|$ & 0.3079176 & 0.3123318 & 0.3034334 & 0.2988 & AVRG & & 81 \\
\hline 36) MA 2-Pentanone & 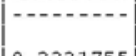 & $\mid--1,-1$ & 0.1914109 & 0.2057837 & 0.2209924 & 0.2204915 & & & & \\
\hline (1) & $\mid 0.2221755$ & $|0.1945759|$ & 0.1987284 & & & & 0.2077 & AVRG & & 6.4331 \\
\hline 37) MCA 1,2-Dichloro & $0.3034973 \mid$ & $\left|\begin{array}{l}0.2970915 \\
0.2782884\end{array}\right|$ & $\left|\begin{array}{|c|}0.3016639 \\
0.2809564\end{array}\right|$ & 0.3028971 & 0.3150810 & 0.3112623 & 0.2988 & |AVRG & & 4.3985 \\
\hline 38) MA Methylcyclohexane & .4696647 & $\mid \begin{array}{l}0.4329683 \\
0.4287871\end{array}$ & $\mid$ & 0.4819630 & 0.4905907 & 0.4832619 & 0.4621 & AVRG & & \\
\hline 39) MA Dibromomethane & 2186936 & $\left|\begin{array}{l}\mid-2172094 \\
0.2113588\end{array}\right|$ & .2232545 & 0.2187509 & 0.2217841 & 0.2187688 & & $\mid-\cdots$ & & 57 \\
\hline 多 & 2186936 & $\left|\begin{array}{l}0.2113588 \\
--\ldots-1\end{array}\right|$ & $\left|\begin{array}{l}0.2105964 \\
-\ldots-\ldots\end{array}\right|$ & 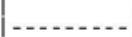 & & & $\mid \begin{array}{l}0.2176 \\
-\ldots-1\end{array}$ & $\mid$ & & 2. \\
\hline 40) MA Bromodichlo & 0.4739910 & $\left|\begin{array}{|l}0.4270206 \\
0.4551744\end{array}\right|$ & $\left|\begin{array}{l}0.4432612 \\
0.4564423\end{array}\right|$ & 0.4455599 & 0.4833777 & 0.4662799 & 0.4564 & AVRG & & 3.9628 \\
\hline $\begin{array}{l}\text { 41) MA } 2 \text {-Chloroethylvinyl ether } \\
0.0039|0.1682| 0.00\end{array}$ & 932301 & $\begin{array}{r}14580 \\
1619380\end{array}$ & $\begin{array}{r}32369 \\
1353645\end{array}$ & 93911 & 200448 & 400193 & & $\begin{array}{l}1 / x \\
\text { LINR }\end{array}$ & & 0.9909 \\
\hline $\begin{array}{l}\text { 42) MA cis-1,3-Dichloropropylen } \\
-0.0052|0.5000| 0.00\end{array}$ & 521523 & $\begin{array}{r}6298 \\
1019621\end{array}$ & $\begin{array}{r}14429 \\
810458\end{array}$ & 40404 & 97152 & 202193 & & \begin{tabular}{|l|l}
$1 / x$ \\
LINR
\end{tabular} & & 993 \\
\hline 44) MA 4-Methyl-2-pentanone & 0.3410195 & $\left|\begin{array}{l}\mid-2951503 \\
0.295 \mid \\
0.2910938\end{array}\right|$ & $\left|\begin{array}{l}\mid-1 .-1 \\
0.3454391 \\
0.3029928\end{array}\right|$ & 0.3805116 & 0.3883504 & 0.3718260 & 0.3395 & |AVRG & & 11.5647 \\
\hline & & & & & & & & $-\cdots$ & $\mid--$ & -....... \\
\hline 45) $\mathrm{S}$ & 1.2459444 & $\left|\begin{array}{l}1.2619693 \\
1.2329948\end{array}\right|$ & $\left|\begin{array}{l}1.2550862 \\
1.2420845\end{array}\right|$ & 1.2365487 & 1.2494165 & 1.2354089 & 1.2449 & AVRG & & 0.8181 \\
\hline 46) MCA Toluene & 1.3837170 & $\left|\begin{array}{l}1.4561231 \\
1.2435658\end{array}\right|$ & $\left|\begin{array}{l}1.5253949 \\
1.2832722\end{array}\right|$ & 1.4928237 & 1.5114965 & 1.4710616 & 1.4209 & AVRG & & 55 \\
\hline trans -1 , & & 5462 & & 34319 & 85687 & 183986 & & $1 / x$ & & \\
\hline$-0.0074|0.5814| 0.00$ & 487522 & 951025 & 757154 & & & & & LINR & & 0.99 \\
\hline
\end{tabular}

VOA1-8260-032618.M Tue Mar 27 01:20:22 2018

Page: 3 
Response Factor Report VOA1

GEL Laboratories. ILC

Method File : C: \msdchem \1\DATA 032618 V1 $\backslash$ VOA1-8260-032618.M

Last Update : Mon Mar $26 \quad 23: 38: 562018$

Integrator : (RTE Integrator)

Response via : Initial Calibration

For Linear Calibration: $x=$ concentration ratio, $y=$ response ratio. $y=b+m 1(x)+m 2(x E 2)$

\begin{tabular}{|c|c|c|c|c|c|c|c|c|c|c|c|}
\hline $\mathrm{b}$ & $\underset{\mathrm{m} 1}{\operatorname{Compound}} \mathrm{m} 2$ & $\begin{array}{l}8 \\
6\end{array}$ & $\begin{array}{l}1 \\
7\end{array}$ & $\begin{array}{l}2 \\
9\end{array}$ & 3 & 4 & 5 & Avg & |Curve & Exp & RSD $/ \mathrm{r} 2$ \\
\hline 48) MA & 1,1,2-Trichloroeth & 0.3160217 & $\begin{array}{l}0.3191595 \\
0.2980786\end{array}$ & $\mid \begin{array}{l}0.3291482 \\
0.2967413\end{array}$ & 0.3164336 & 0.3172934 & 0.3183083 & 0.3139 & |AVRG & & \\
\hline 49) MA & 2-Hexanone & 0.3144514 & 0.3204271 & $\mid \begin{array}{l}0.3619644 \\
0.2688154\end{array}$ & 0.3869478 & 0.4019146 & 0.3498717 & 0.3435 & AVRG & & 13.3602 \\
\hline 50) $\mathrm{MA}$ & 1,3-Dichloropropane & 0.5815879 & $\mid \begin{array}{l}0.5521146 \\
0.5233528\end{array}$ & $\begin{array}{l}0.5958101 \\
0.5318734\end{array}$ & 0.5833698 & 0.6049761 & 0.6021777 & $\ldots$ & $-\cdots$ & & 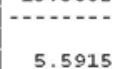 \\
\hline & 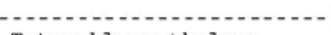 & & 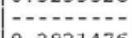 & $-\ldots$ & & & & & $\cdots$ & & \\
\hline 51) $M A$ & Tetrachlor & 0.2911156 & $\begin{array}{l}0.2921476 \\
0.2719161\end{array}$ & $\begin{array}{l}0.3031483 \\
0.2762948\end{array}$ & 0.2979091 & 0.2961915 & 0.2968246 & 0.2907 & AVRG & & 3.7621 \\
\hline 52) MA & Dibromochloromethane & $\mid \begin{array}{l}0.4872295 \\
0.48\end{array}$ & $\mid \begin{array}{l}0.3937347 \\
0.396 \\
0.4746762\end{array}$ & $\mid \begin{array}{l}0.0-1 \\
0.4263629 \\
0.4727962\end{array}$ & $|0.4352102|$ & 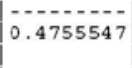 & $|0.4725781|$ & $\mid \begin{array}{l}0 .-.-1 \\
0.4548\end{array}$ & AVRG & & 7.1682 \\
\hline 53) MA & 1,2-Dibromoethane & & 0.3298043 & 0.3562277 & 0.3693205 & 0.3798708 & 0.3859035 & & & i. & \\
\hline & & 3932172 & 0.3799075 & 0.37 & & & & 0.3716 & AVRG & & 58 \\
\hline 54) MPA & Chlorobenze & 1.0373318 & $\begin{array}{l}0.1397324 \\
0.9484544\end{array}$ & $\begin{array}{l}1.1402680 \\
0.9595364\end{array}$ & 1.1035490 & 1.1160162 & 1.0933229 & 1.0673 & AVRG & & 7.2205 \\
\hline 55) $\mathrm{MA}$ & $1,1,1,2$-Tetrachlorcethan & & 0.3580469 & 0.3822894 & 0.3935022 & 0.4198595 & 0.4254237 & & & & \\
\hline & & 0.4218871 & 0.3958199 & 0.4040116 & & & & 0.4001 & AVRG & & 5.7222 \\
\hline 56) МCA & Ethylbenze & 1.6149805 & $\begin{array}{l}1.5531570 \\
1.4018590\end{array}$ & $\begin{array}{l}1.7074565 \\
1.4489627\end{array}$ & 1.7425911 & 1.7598521 & 1.7434554 & 1.6215 & AVRG & & 8.6837 \\
\hline 57) $\mathrm{MA}$ & m,p-Xylenes & 0.6279568 & $\mid \begin{array}{l}0.6042003 \\
0.5394887\end{array}$ & $\mid \begin{array}{l}0.6566310 \\
0.5692736\end{array}$ & 0.6835910 & 0.6886433 & 0.6770918 & $\mid \begin{array}{l}0 . \\
0.6309\end{array}$ & $\mid \begin{array}{l}\cdots--- \\
\text { AVRG }\end{array}$ & |-- & 8.8423 \\
\hline 58) $\mathrm{MA}$ & o-xylene & 0.6646380 & $\mid \begin{array}{l}0.5258410 \\
0.5824471\end{array}$ & $\begin{array}{l}0.6246785 \\
0.6107119\end{array}$ & $\mid 0.6633841$ & 0.7112564 & $\mid 0.7063249$ & 0.6362 & AVRG & & 9.9328 \\
\hline 59) MA & Styrene & $\mid 1.1057995$ & $\mid \begin{array}{l}0.8874567 \\
0.9366081\end{array}$ & $\begin{array}{l}0.0725693 \\
1.0720 \\
0.9920923\end{array}$ & $\mid$ & 1.2161062 & 1.1946899 & 1.0693 & AVRG & & 11.2663 \\
\hline 61) MPA & Bromoform & 0.5559974 & $\mid \begin{array}{l}0.4256759 \\
0.5513774\end{array}$ & $\mid \begin{array}{l}- \\
0.4246603 \\
0.5411837\end{array}$ & $\mid$ & 0.4996311 & $\mid-5224546$ & 0.4981 & $\cdots$ & - & 10.8611 \\
\hline 62) MA & Isopropylbenzene & 2.7268550 & $\begin{array}{l}2.0347607 \\
2.4477411\end{array}$ & $\begin{array}{l}-1.4079251 \\
2.5129989\end{array}$ & 2.6640927 & 2.8976395 & $\left|\begin{array}{l}\mid-8572279 \\
2.85\end{array}\right|$ & 2.5687 & AVRG & $\mid-\cdots$ & 10.9545 \\
\hline 63) SA & Bromofluorobe & $|0.9701649|$ & $\begin{array}{l}0.9694472 \\
0.9563591\end{array}$ & $\begin{array}{l}0.9490863 \\
0.9682008\end{array}$ & 0.9597598 & 0.9618963 & 0.9466911 & $\mid \begin{array}{l}0.9602 \\
0.960\end{array}$ & |AVRG & & 0.94 \\
\hline
\end{tabular}

Page: 4 
Response Factor Report VOA1

GEL Laboratories. ILC

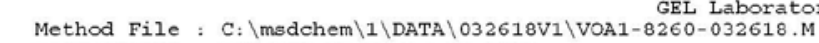

Method File: C: $\backslash$ msdchem $\backslash 1 \backslash$ DATA $\backslash 032618$
Last Update : Mon Mar 26 23:38:56 2018

Last Update : Mon Mar $26 \quad 23: 38:$

Response via : Initial Calibration

For Linear Calibration: $x=$ concentration ratio, $y=$ response ratio. $y=b+m 1(x)+m 2(x E 2)$

\begin{tabular}{|c|c|c|c|c|c|c|c|c|c|c|c|}
\hline $\mathrm{b}$ & | ${ }_{\mathrm{m} 1}^{\text {Compound }} \mid \mathrm{m} 2$ & $\begin{array}{l}8 \\
6\end{array}$ & $\begin{array}{l}1 \\
7\end{array}$ & $\begin{array}{l}2 \\
9\end{array}$ & 3 & 4 & 5 & Avg & |Curve & Exp & : $\mathrm{RSD} / \mathrm{r} 2$ \\
\hline 64) MPA & $1,1,2,2$-Tetrachloroe & 330 & 0.8570408 & 0.8744984 & 0.8664137 & 0.8895408 & $|0.8912432|$ & 0.8657 & JVVR & & \\
\hline & . & 年 & - & 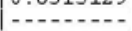 & & & & & 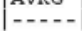 & & \\
\hline 65) $M A$ & 1,2,3-Trichloropro & 0.2361496 & $\mid \begin{array}{l}0.2138859 \\
0.2276857\end{array}$ & 0.2208134 & 0.2329221 & 0.2314735 & $|0.2313724|$ & 0.2278 & AVRG & & 688 \\
\hline 66) MA & & & & 7822950 & 0.8099807 & 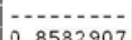 & & & & & \\
\hline 66) MA & Bromobenzene & 0.8364175 & 0.7942752 & 0.8014384 & 10.8099807 & 10.8582907 & $|0.8466472|$ & 0.8135 & AVRG & & 3.7087 \\
\hline 67) MA & n-Propylber & $\mid \begin{array}{l}\mid-1 . \\
3.3059018\end{array}$ & 3.0686591 & 3.3404959 & 3.5573534 & 3.6398225 & $\mid$ & 3.2853 & AVRG & & \\
\hline 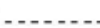 & $\ldots$ & $\mid \begin{array}{l}3.3059018 \\
-\end{array}$ & |2.8470881 & 2.9538000 & $-\ldots-1-1$ & 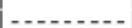 & -1 & 13.2853 & AVKG & & \\
\hline 68) $\mathrm{MA}$ & 1,3,5-Trimethylbenze & 2.4391922 & $\begin{array}{l}2.0906525 \\
2.1457031\end{array}$ & $\begin{array}{l}2.3899197 \\
2.2368376\end{array}$ & 2.5560805 & 2.6737756 & $2.5807291 \mid$ & 2.3891 & AVRG & & 444 \\
\hline 69) MA & 2-Chlorotoluene & & 0.6272 & 0.6886570 & 0.7498822 & 0.7898752 & 0.7777139 & & -- & & \\
\hline & & 0.7550611 & 0.7031823 & $\mid \begin{array}{l}0.6886570 \\
0.7085021\end{array}$ & & & & 0.7250 & AVRG & & 7.3960 \\
\hline 70) MA & 4-Chlorotol & $\left|\begin{array}{l}\cdots \\
2.2554417\end{array}\right|$ & $\mid \begin{array}{l}0.0964413 \\
2.0345326\end{array}$ & $\mid \begin{array}{l}0.3190853 \\
2.0888216\end{array}$ & 2.3567927 & 2.4602481 & 2.3642754 & 2. & AVRG & & 6.9 \\
\hline &.--1 & (2) & & . & 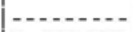 & & & & & & \\
\hline 71) $M A$ & tert-Butyl & 0.4799509 & $\begin{array}{l}0.3149902 \\
0.4531909\end{array}$ & $\begin{array}{l}0.3567443 \\
0.4655773\end{array}$ & 0.4335593 & 0.4688922 & 0.47 & 0.4309 & AVRG & & 14.2451 \\
\hline 72) $\mathrm{MA}$ & 1,2,4-Trime & 2.5487023 & $\begin{array}{l}2.0976390 \\
2.2711639\end{array}$ & 2.4832609 & 2.6423614 & 2.8103758 & $\mid$ & 2.4933 & AVRG & & 9 \\
\hline & - . & $-\ldots$ & & - & & & & $\ldots$ & $-\ldots$ & -- & $\ldots$ \\
\hline 73) $\mathrm{MA}$ & sec-Butylben: & 3.0330065 & $\begin{array}{l}2.4629318 \\
2.6534113\end{array}$ & $\begin{array}{l}2.7798059 \\
2.7714248\end{array}$ & 3.0808954 & 3.3210051 & 3.2354251 & 2.9172 & AVRG & & 10.2067 \\
\hline 74) MA & 4 -Isopropyl to & 2.7032056 & $\begin{array}{l}-1.0433441 \\
2.3988263\end{array}$ & $\begin{array}{l}2.4445790 \\
2.4833061\end{array}$ & 2.7034871 & 2.9751984 & 2.8799888 & 2.5790 & AVRG & & 11.5872 \\
\hline 75) MA & 1,3-Dichlorobenzene & & 450 & -2 & 1.6613094 & 1.7008209 & 1.6640376 & & & & \\
\hline 75) MA & 1,3-Dichlorobenzene & $\mid 1.5828907$ & $\begin{array}{l}1.6296450 \\
1.4527441\end{array}$ & $\begin{array}{l}1.6899352 \\
1.4875639\end{array}$ & 1.6613094 & 11.7008209 & $1.6640376 \mid$ & 1.6086 & AVRG & & 8039 \\
\hline 76$) 18$ & 4 & (2) & & & 1,6737158 & 17217345 & 16280854 & & & & \\
\hline 76) $M A$ & ichloro & 1.5742424 & $\begin{array}{l}1.6964157 \\
1.4416282\end{array}$ & $\begin{array}{l}1.7256986 \\
1.4827836\end{array}$ & 1.6737158 & 1.7217345 & $|1.6289854|$ & 1.6182 & AVRG & & 6.7309 \\
\hline 77) $M A$ & n-Butylbenzene & $2.6329742 \mid$ & $\begin{array}{l}2.0757813 \\
2.2581949\end{array}$ & $\begin{array}{l}2.4428477 \\
2.3987513\end{array}$ & 2.7271519 & 2.9443192 & 2.8518680 & 2.5415 & AVRG & & 11.7918 \\
\hline 78) MA & 1,2-Dichlorobenzene & 1.5673318 & $\begin{array}{l}1.4917120 \\
1.4392768\end{array}$ & $\begin{array}{l}1.5405992 \\
1.4822692\end{array}$ & 1.5786739 & 1.6605176 & 1.6217988 & 1.5478 & AVRG & & 4.8092 \\
\hline
\end{tabular}

VOA1-8260-032618.M Tue Mar 27 01:20:22 2018

Page: 5 
Method File GEL Laboratories, LLC

Method File : C: Imsdchem\1\DATA 032618V1 \VOA1-8260-032618.M

Last Update : Mon Mar 26 23:38:56 2018

Integrator : (RTE Integrator)

Response via : Initial Calibration

For Linear Calibration: $x=$ concentration ratio, $y=$ response ratio. $y=b+m 1(x)+m 2(x E 2)$

\begin{tabular}{|c|c|c|c|c|c|c|c|c|c|c|}
\hline | Compound & $\begin{array}{l}8 \\
6\end{array}$ & $\frac{1}{7}$ & $\begin{array}{l}2 \\
9\end{array}$ & 3 & 4 & 5 & Avg & |Curve & Exp & ?RSD / r2 \\
\hline $\begin{array}{l}\text { 79) MA } 1,2 \text {-Dibromo- } 3 \text {-chloroprop } \\
-0.0025|0.1759| 0.00\end{array}$ & 84567 & $\begin{array}{r}1046 \\
180322\end{array}$ & $\begin{array}{r}2336 \\
139517\end{array}$ & 6249 & 14472 & 29862 & & $\begin{array}{l}1 / x \\
\text { LINR }\end{array}$ & \# & \\
\hline 80) MA 1,2,4-Trichlorobenzene & & 0.9327941 & 0.9626457 & 1.0412916 & 1.2085487 & 1.1452914 & & $\ldots$ & & \\
\hline 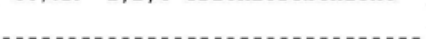 & 1.1644162 & 1.0857907 & 1.1055839 & & & & 1.0808 & AVRG & & 8.9526 \\
\hline 81) MA Hexachlorobutadiene & 0.6993255 & $\left|\begin{array}{l}0.6485444 \\
0.6784818\end{array}\right|$ & $\mid \begin{array}{l}0.6492331 \\
0.6718937\end{array}$ & 0.6620304 & 0.7429349 & 0.6958988 & 0.6810 & AVRG & & 4.6118 \\
\hline 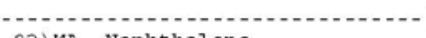 & ( & - - - & - - - & ----1 & 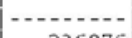 & 450900 & & & & \\
\hline $\begin{array}{l}\text { 82) MA Naphthalene } \\
\begin{array}{ll|l|l|}-0.0206 \mid & 2.2757 \mid & 0.00\end{array}\end{array}$ & 1171488 & $\begin{array}{r}13396 \\
2192811\end{array}$ & $\begin{array}{r}31378 \\
1781292\end{array}$ & 93416 & 236876 & 459984 & & $\begin{array}{l}1 / x \\
\text { LINR }\end{array}$ & & 0.9986 \\
\hline 83) MA 1,2,3-Trichlorobenzene & $\left|\begin{array}{ll}0 . \\
0.9974045\end{array}\right|$ & $\left|\begin{array}{l}\mid-1-1 \\
0.8652249 \\
0.9508840\end{array}\right|$ & $\left|\begin{array}{l}0.9502794 \\
0.9594492\end{array}\right|$ & $|0.9033509|$ & 1.0611909 & $\mid-0012692$ & 0.9611 & AVRG & & 6 \\
\hline 85) B Acrolein & & 2963 & 7103 & 20728 & 44824 & 87195 & & $1 / x$ & & \\
\hline $\begin{array}{lllll}-0.0020 \mid & 0.0464 \mid 0.00\end{array}$ & 232141 & 466790 & & & & & & LINR & $\#$ & 0.9996 \\
\hline 86) B Trichlorotrifluoroethane & 0.1003098 & 0.1024484 & 0.1090057 & 0.1066670 & 0.0988205 & 0.1034477 & 0.1020 & AVRG & & 5.1829 \\
\hline $\begin{array}{l}\text { 87) B Isopropyl Alcohol } \\
-0.0106|0.0165| 0.00\end{array}$ & 834124 & $\left|\begin{array}{r}11504 \\
1682244\end{array}\right|$ & 22264 & 58751 & 130673 & 297212 & & $\begin{array}{l}--\cdot- \\
1 / x \\
\text { LINR }\end{array}$ & $t$ & 0.9961 \\
\hline 88) B Allyl chloride & .1405864 & $\left|\begin{array}{|c|}\mid-1100411 \\
0.1272786\end{array}\right|$ & 0.1229206 & $|0.1331931|$ & 0.1293906 & $|0.1417796|$ & 0.1293 & AVRG & & 8.4358 \\
\hline 89) B tert-Butyl Alcohol & .0190558 & 0.0134528 & 0.0133320 & 0.0139910 & 0.0151711 & $\left|\begin{array}{ll}0.0168904 \\
0.010\end{array}\right|$ & 0.0153 & AVKG & & 14.7883 \\
\hline & & & & & & & & & & \\
\hline Acrylonitrile & 0.1062916 & $\left|\begin{array}{l}0.0876873 \\
0.0993693\end{array}\right|$ & 0.0965140 & 0.0998718 & 0.1014475 & $|0.1056930|$ & 0.0996 & AVRG & & 6.3113 \\
\hline 91) B Isopropyl ether & .9063240 & $\left|\begin{array}{l}0.6172967 \\
0.8408697\end{array}\right|$ & 0.6582282 & $|0.7210710|$ & 0.7561732 & $|0.8519489|$ & 0.7646 & AVRG & & 13.9764 \\
\hline 92) B 2-Chloro-1,3-butadiene & .3668768 & $\mid \begin{array}{l}0.3081053 \\
0.3401195\end{array}$ & 0.2962253 & $\mid$ & 0.3204414 & 0.3557869 & $0 .-100$ & ANR & $-\ldots$ & 13.9704 \\
\hline $\begin{array}{c}\text { 93) B Ethyl tert-butyl ether } \\
-0.0091 \\
-0.6811\end{array}$ & 698562 & $\begin{array}{r}8894 \\
1381838\end{array}$ & 18347 & 49269 & 105770 & 244734 & & $\begin{array}{l}1 / x \\
\text { LINR }\end{array}$ & & 0.9960 \\
\hline 94$) \mathrm{B} \quad$ Ethyl acetate & 0.2512262 & $\left|\begin{array}{l}0.2115318 \\
0.2172888\end{array}\right|$ & 0.2253938 & $|0.2379592|$ & 0.2435703 & $\mid 0.2546387$ & 0.2345 & AVRG & & 7.14 \\
\hline
\end{tabular}

VOA1-8260-032618.M Tue Mar 27 01:20:22 2018

Page: 6 
Response Factor Report VOA1

GRL Iaboratories. LLC

GEL Laborato
Method File : C: $\backslash$ msdchem $\backslash 1 \backslash$ DATA $\backslash 032618 V 1 \backslash$ VOA1-8260-032618.M

Method File: C: $\backslash$ msdchem $\backslash 1 \backslash$ DATA $\backslash 032618$ Mpdate : Mon Mar 26 23:38:56 2018

Last Update : Mon Mar $26 \quad 23: 38$ :
Integrator : (RTE Integrator)

Response via : Initial Calibration

For Linear Calibration: $x=$ concentration ratio, $y=$ response ratio. $y=b+m 1(x)+m 2(x E 2)$

\begin{tabular}{|c|c|c|c|c|c|c|c|c|c|c|c|}
\hline $\mathrm{b}$ & | ${ }_{\mathrm{m} 1}^{\text {Compound }}$ | $\mathrm{m} 2$ & $\begin{array}{l}8 \\
6\end{array}$ & $\begin{array}{l}1 \\
7\end{array}$ & $\begin{array}{l}2 \\
9\end{array}$ & 3 & 4 & 5 & Avg & Curve & Exp & : $\mathrm{RSD} / \mathrm{r} 2$ \\
\hline 95) B & Propionitrile & $|0.0424080|$ & $\left|\begin{array}{l}0.0366310 \\
0.0390556\end{array}\right|$ & 0.0371281 & 0.0403405 & 0.0408970 & 0.0418666 & 0.0398 & AVRG & & 5.6511 \\
\hline 96) B & Methacrylonitrile & 0.1549908 & $\mid \begin{array}{l}0.1251181 \\
0.1380200\end{array}$ & 0.1414016 & 0.1519758 & 0.1548330 & 0.1593788 & 0.1465 & AVRG & & 8.3232 \\
\hline-1 & 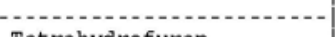 & $-\ldots$ & - & 0700096 & 1 & 101700 & - - - & & & & \\
\hline 97) B & Tetrahydrofuran & 0.0830650 & $\begin{array}{l}0.0811905 \\
0.0763305\end{array} \mid$ & 0.0798986 & 0.0821157 & 0.0814788 & 0.0830828 & 0.0810 & AVRG & & 2.9000 \\
\hline 98) B & Isobutyl alcohol & 0.0104949 & $\mid \begin{array}{l}0.0098390 \\
0.0092240\end{array}$ & 0.0102006 & $|0.0105823|$ & $\mid \begin{array}{l}0.0109717 \\
0.010\end{array}$ & $\mid \begin{array}{l}0.0107074 \\
0.010\end{array}$ & 0.0103 & AVRG & \$ & 5.7694 \\
\hline $\begin{array}{l}\text { 99) } \mathrm{B} \\
-0.0\end{array}$ & Methyl tert-amyl ether & 515322 & $\begin{array}{r}6940 \\
1040511\end{array}$ & 14107 & 37975 & 79697 & 180758 & & $\begin{array}{l}--\cdot-- \\
1 / x \\
\text { LINR }\end{array}$ & & 0.9959 \\
\hline 100$) \mathrm{B}$ & Methyl methacrylate & 1935963 & $\mid \begin{array}{l}-1427820 \\
0.1699824 \\
0.1699\end{array}$ & 0.1651882 & $\mid-1827359$ & $\mid-1912556$ & 0.2009976 & $\mid \begin{array}{ll}\cdots \\
0.1781\end{array}$ & $-\cdots$ & & 11.3219 \\
\hline & & & - & & & & & $-\ldots$ & & & 2.1 \\
\hline 101) B & 1,4-Dioxane & .0027558 & $\begin{array}{l}0.0021821 \\
0.0025829\end{array} \mid$ & 0.0024677 & 0.0024497 & 0.0026365 & 0.0027536 & 0.0025 & AVRG & \# & 7.9266 \\
\hline $\begin{array}{l}102) \mathrm{B} \\
-0.0\end{array}$ & 089 | $0.0867 \mid \quad 0.00$ & 452790 & $\begin{array}{r}3384 \\
867707\end{array}$ & 7721 & 25243 & 63644 & 154606 & & $\begin{array}{l}-\cdot-- \\
1 / x \\
\text { LINR }\end{array}$ & & 0.9944 \\
\hline 104$) \mathrm{B}$ & Ethyl methacrylate & 0.4612967 & $\mid \begin{array}{l}0.3503388 \\
0.3745463\end{array}$ & 0.4185258 & 0.4702953 & 0.4901750 & 0.5023455 & $\mid 0.4382$ & AVRG & & 13.3510 \\
\hline 106) B & 1-Chlorohexane & 0.5262915 & $\mid \begin{array}{l}0.3591702 \\
0.4682047\end{array}$ & 0.4115871 & $\mid \begin{array}{ll}0.4589233 \\
0 .\end{array}$ & 0.4776681 & 0.5153146 & 0.4596 & AVRG & & 12.6698 \\
\hline 107) B & cis-1, 4-Dichloro-2 -buten & 0.2582613 & $\mid \begin{array}{l}0.1903345 \\
0.2176661\end{array}$ & 0.2160687 & $\left|\begin{array}{ll}0.2524249 \\
0.25\end{array}\right|$ & 0.2587272 & 0.2654956 & 0.2370 & AVRG & & 12.1362 \\
\hline 108$) \mathrm{B}$ & Cyclohexanone & 0.0218646 & $\mid \begin{array}{l}0.0169351 \\
0.0215455\end{array}$ & 0.0157901 & 0.0177005 & 0.0202711 & $\mid 0.0211931$ & 0.0193 & AVRG & & 12.7801 \\
\hline 109$) \mathrm{B}$ & trans-1,4-Dichloro-2-but & 0.2392125 & $\begin{array}{l}0.2063915 \\
0.1999553\end{array}$ & 0.2282491 & 0.2473969 & 0.2529514 & 0.2519142 & 0.2323 & AVRG & D. & 9.3339 \\
\hline 110$) \mathrm{B}$ & Pentachloroethane & 0.4453783 & $\begin{array}{l}0.3652509 \\
0.3751834\end{array}$ & $\left|\begin{array}{ll}- \\
0.4053070\end{array}\right|$ & $\mid 0.4207437$ & 0.4323936 & 0.4651170 & 0.4156 & AVRG & & 8.7424 \\
\hline $\begin{array}{l}\text { 111) B } \\
-0.0\end{array}$ & $\begin{array}{l}\text { Benzyl chloride } \\
732 \mid \text { | } 1.1790 \mid 0.00\end{array}$ & 2777401 & 28820 & 68408 & 223009 & 514537 & 1136181 & & \begin{tabular}{|l|}
$1 / x$ \\
LINR
\end{tabular} & & 0.99 \\
\hline
\end{tabular}

VOA1-8260-032618.M Tue Mar 27 01:20:22 2018

Page: 7 
Response Factor Report VOAI

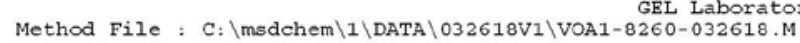

Last Update : Mon Mar 26 23:38:56 2018

Integrator : (RTE Integrator)

Response via : Initial Calibration

For Linear Calibration: $x=$ concentration ratio, $y=$ response ratio. $y=b+m 1(x)+m 2(x E 2)$

\begin{tabular}{|c|c|c|c|c|c|c|c|c|c|c|c|}
\hline b & $\underset{\mathrm{m} 1}{\text { Compound }} \mathrm{m} 2$ & $\begin{array}{l}8 \\
6\end{array}$ & $\frac{1}{7}$ & $\begin{array}{l}2 \\
9\end{array}$ & 3 & 4 & 5 & Avg & |Curve| & Exp & 8RSD/r2 \\
\hline $\begin{array}{c}112) \text { B bis } \\
-0.0189 \text { । }\end{array}$ & $\left\{\begin{array}{l}(2-\text { Chloroi sopropy } 1) \text { et } \\
0.2810 \mid 0.00\end{array}\right.$ & 717726 & $\begin{array}{r}7165 \\
1293820\end{array}$ & 15711 & $\begin{array}{r}-28344 \\
4834\end{array}$ & $\begin{array}{r}115319 \\
11531\end{array}$ & $\begin{array}{r}-265092 \\
265\end{array}$ & & $\mid$\begin{tabular}{l|}
$-\cdots$ \\
$1 / x$ \\
LINR
\end{tabular} & $\begin{array}{r}\cdots \\
\#\end{array}$ & 0.9964 \\
\hline
\end{tabular}

(\#) = Out of Range (\$) = Individual RF Out of Range

AVRG $=$ Average, LINR $=$ Linear Regression, $1 / x=$ the inverse of concentration, $1 / x^{\wedge} 2=$ the inverse square of concentration 
Continuing Calibration Summary

Instrument ID: VOA1.I

Data File: $\quad 032618 \mathrm{~V} 1 \backslash 1 \mathrm{~B} 121 . \mathrm{D}$

Lab Sample ID W1VM180326-10

Quant Type ISTD
Client SDG: $\quad 446517$

Injection Date: $\quad$ 26-MAR-18 23:15

Init. Cal. Date(s) : 26-MAR-18 14:10 - 26-MAR-18 18:5

Method: $\quad 032618 \mathrm{~V} 1 \backslash$ VOA1-8260-032618.M

Method Update: $\quad$ 26-MAR-18 23:38

\begin{tabular}{|c|c|c|c|c|c|c|c|c|c|}
\hline Compound & $\begin{array}{c}\text { AVERF / } \\
\text { Amount }\end{array}$ & $\begin{array}{c}\mathrm{RF} \\
\mathrm{CCV}\end{array}$ & $\begin{array}{c}\text { Nominal } \\
\mathrm{CCV}\end{array}$ & Min RF & $\begin{array}{c}\text { RF } \\
Q\end{array}$ & $\begin{array}{c}\text { \%D / } \\
\text { \%Drift }\end{array}$ & Max & $\begin{array}{c}\text { Drift } \\
\text { Q }\end{array}$ & $\begin{array}{l}\text { Curve } \\
\text { Type }\end{array}$ \\
\hline S 1,2-Dichloroethane-d4 & 0.1636 & 0.1612 & & .01 & & -1.46699 & 60 & & Averaged \\
\hline SToluene-d8 & 1.2449 & 1.2386 & & .01 & & -0.50606 & 60 & & Averaged \\
\hline SBromofluorobenzene & 0.9602 & 0.97631 & & .01 & & 1.67778 & 60 & & Averaged \\
\hline Chloromethane & 0.226 & 0.21849 & & .1 & & -3.32301 & 60 & & Averaged \\
\hline Vinyl chloride & 0.2252 & 0.22194 & & .01 & & -1.4476 & 20 & & Averaged \\
\hline 1,1-Dichloroethylene & 0.4123 & 0.43896 & & .01 & & 6.46617 & 20 & & Averaged \\
\hline 1,1-Dichloroethane & 0.5082 & 0.53799 & & .1 & & 5.86187 & 60 & & Averaged \\
\hline Chloroform & 0.588 & 0.57971 & & .01 & & -1.40986 & 20 & & Averaged \\
\hline Benzene & 1.107 & 1.11371 & & .01 & & 0.60614 & 60 & & Averaged \\
\hline 1,2-Dichloropropane & 0.2988 & 0.29932 & & .01 & & 0.17403 & 20 & & Averaged \\
\hline Toluene & 1.4209 & 1.45611 & & .01 & & 2.47801 & 20 & & Averaged \\
\hline Chlorobenzene & 1.0673 & 1.07511 & & .3 & & 0.73175 & 60 & & Averaged \\
\hline Ethylbenzene & 1.6215 & 1.67893 & & .01 & & 3.54178 & 20 & & Averaged \\
\hline m,p-Xylenes & 0.6309 & 0.65582 & & .01 & & 3.94991 & 60 & & Averaged \\
\hline o-Xylene & 0.6362 & 0.68121 & & .01 & & 7.07482 & 60 & & Averaged \\
\hline Bromoform & 0.4981 & 0.52299 & & .1 & & 4.99699 & 60 & & Averaged \\
\hline 1,1,2,2-Tetrachloroethane & 0.8657 & 0.8293 & & .3 & & -4.20469 & 60 & & Averaged \\
\hline
\end{tabular}

Page 41 of 94 
Continuing Calibration Summary

Instrument ID: VOA1.I

Data File: $\quad 032918 \mathrm{~V} 1 \backslash 1 \mathrm{~B} 402 . \mathrm{D}$

Lab Sample ID W1VM180329-01

Quant Type ISTD
Client SDG: $\quad 446517$

Injection Date: $\quad$ 29-MAR-18 10:16

Init. Cal. Date(s) : 26-MAR-18 14:10 - 26-MAR-18 18:5

Method: $\quad 032618 \mathrm{~V} 1 \backslash$ VOA1-8260-032618.M

Method Update: $\quad$ 26-MAR-18 23:38

\begin{tabular}{|c|c|c|c|c|c|c|c|c|c|}
\hline Compound & $\begin{array}{c}\text { AVERF / } \\
\text { Amount }\end{array}$ & $\begin{array}{c}\mathrm{RF} \\
\mathrm{CCV}\end{array}$ & $\begin{array}{c}\text { Nominal } \\
\mathrm{CCV}\end{array}$ & Min RF & $\begin{array}{c}\text { RF } \\
\text { Q }\end{array}$ & $\begin{array}{c}\text { \%D / } \\
\text { \%Drift }\end{array}$ & Max & $\begin{array}{c}\text { Drift } \\
\text { Q }\end{array}$ & $\begin{array}{l}\text { Curve } \\
\text { Type }\end{array}$ \\
\hline S 1,2-Dichloroethane-d4 & 0.1636 & 0.15646 & & .01 & & -4.3643 & 60 & & Averaged \\
\hline SToluene-d8 & 1.2449 & 1.22346 & & .01 & & -1.72223 & 60 & & Averaged \\
\hline SBromofluorobenzene & 0.9602 & 1.00742 & & .01 & & 4.91773 & 60 & & Averaged \\
\hline Chloromethane & 0.226 & 0.21436 & & .1 & & -5.15044 & 60 & & Averaged \\
\hline Vinyl chloride & 0.2252 & 0.23758 & & .01 & & 5.49734 & 20 & & Averaged \\
\hline 1,1-Dichloroethylene & 0.4123 & 0.34715 & & .01 & & -15.8016 & 20 & & Averaged \\
\hline 1,1-Dichloroethane & 0.5082 & 0.44711 & & .1 & & -12.02086 & 60 & & Averaged \\
\hline Chloroform & 0.588 & 0.5173 & & .01 & & -12.02381 & 20 & & Averaged \\
\hline Benzene & 1.107 & 0.95406 & & .01 & & -13.81572 & 60 & & Averaged \\
\hline 1,2-Dichloropropane & 0.2988 & 0.26441 & & .01 & & -11.50937 & 20 & & Averaged \\
\hline Toluene & 1.4209 & 1.25791 & & .01 & & -11.4709 & 20 & & Averaged \\
\hline Chlorobenzene & 1.0673 & 0.93118 & & .3 & & -12.75368 & 60 & & Averaged \\
\hline Ethylbenzene & 1.6215 & 1.48234 & & .01 & & -8.58218 & 20 & & Averaged \\
\hline $\mathrm{m}, \mathrm{p}$-Xylenes & 0.6309 & 0.57559 & & .01 & & -8.76684 & 60 & & Averaged \\
\hline o-Xylene & 0.6362 & 0.59971 & & .01 & & -5.73562 & 60 & & Averaged \\
\hline Bromoform & 0.4981 & 0.5175 & & .1 & & 3.8948 & 60 & & Averaged \\
\hline 1,1,2,2-Tetrachloroethane & 0.8657 & 0.83671 & & .3 & & -3.34874 & 60 & & Averaged \\
\hline
\end{tabular}

Page 42 of 94 


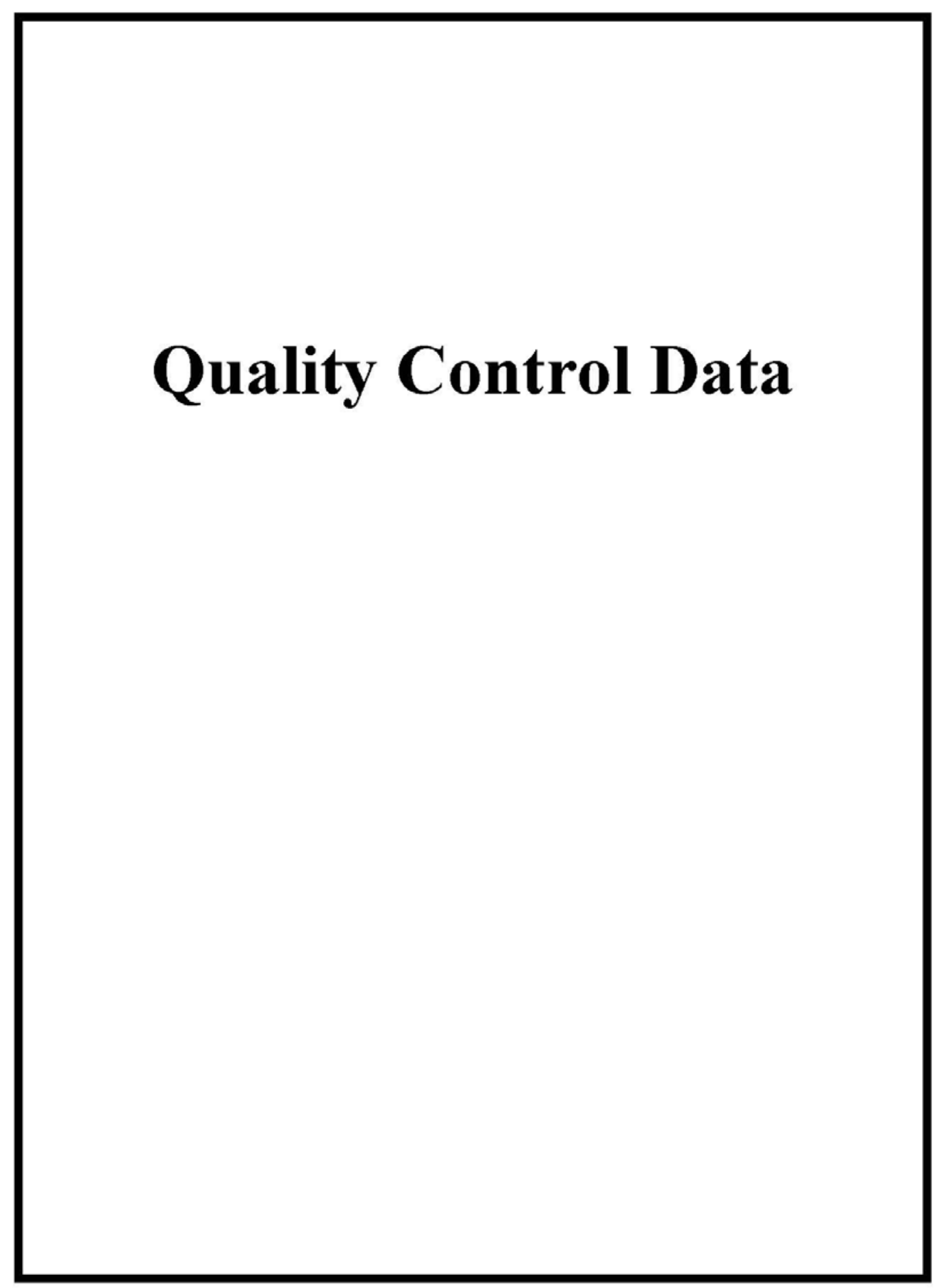

Page 43 of 94 


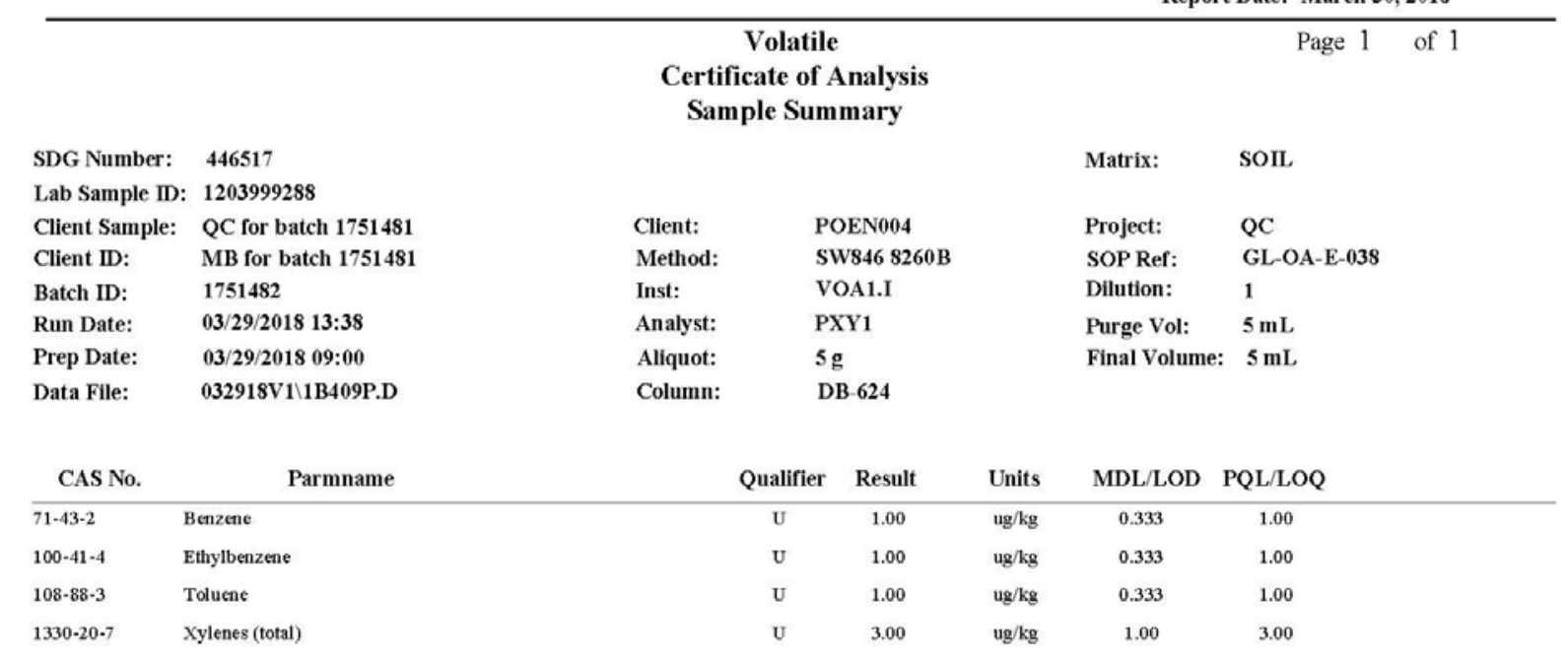

Page 44 of 94 


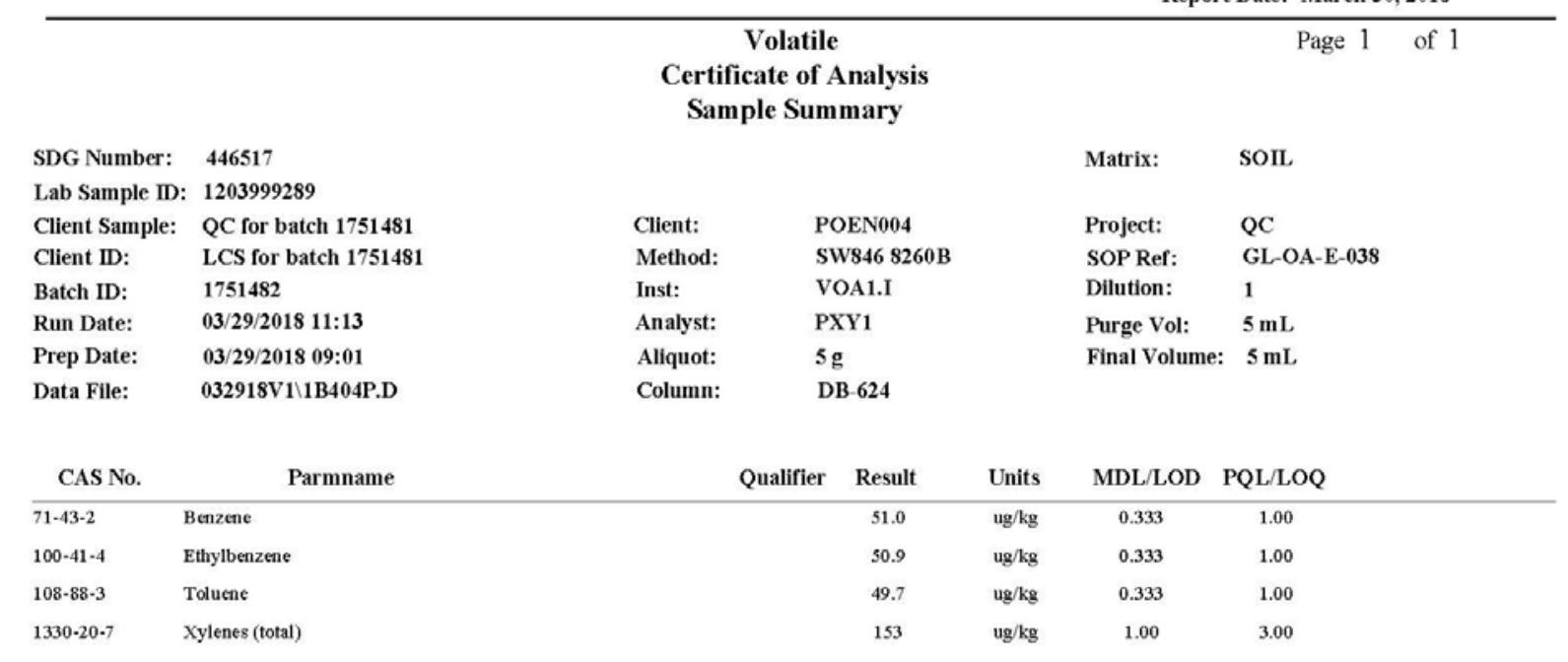

Page 45 of 94 


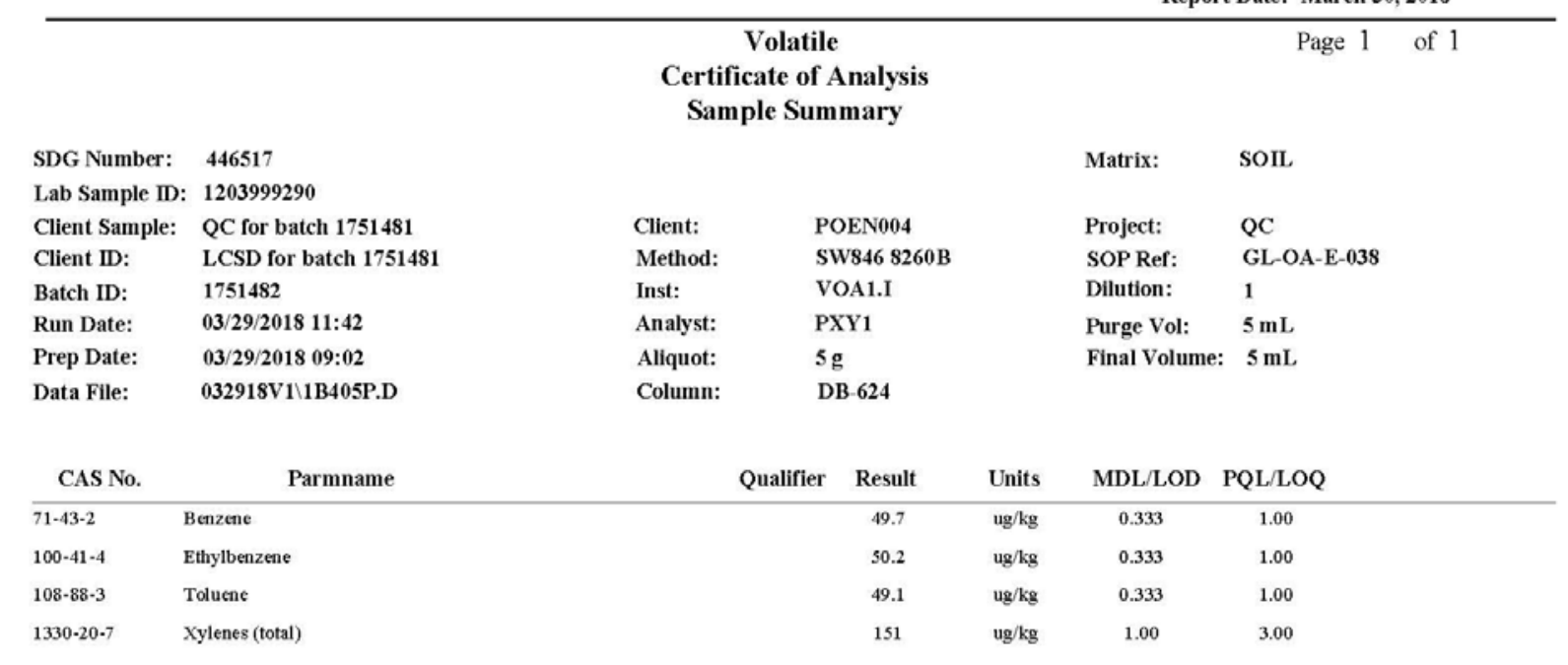

Page 46 of 94 


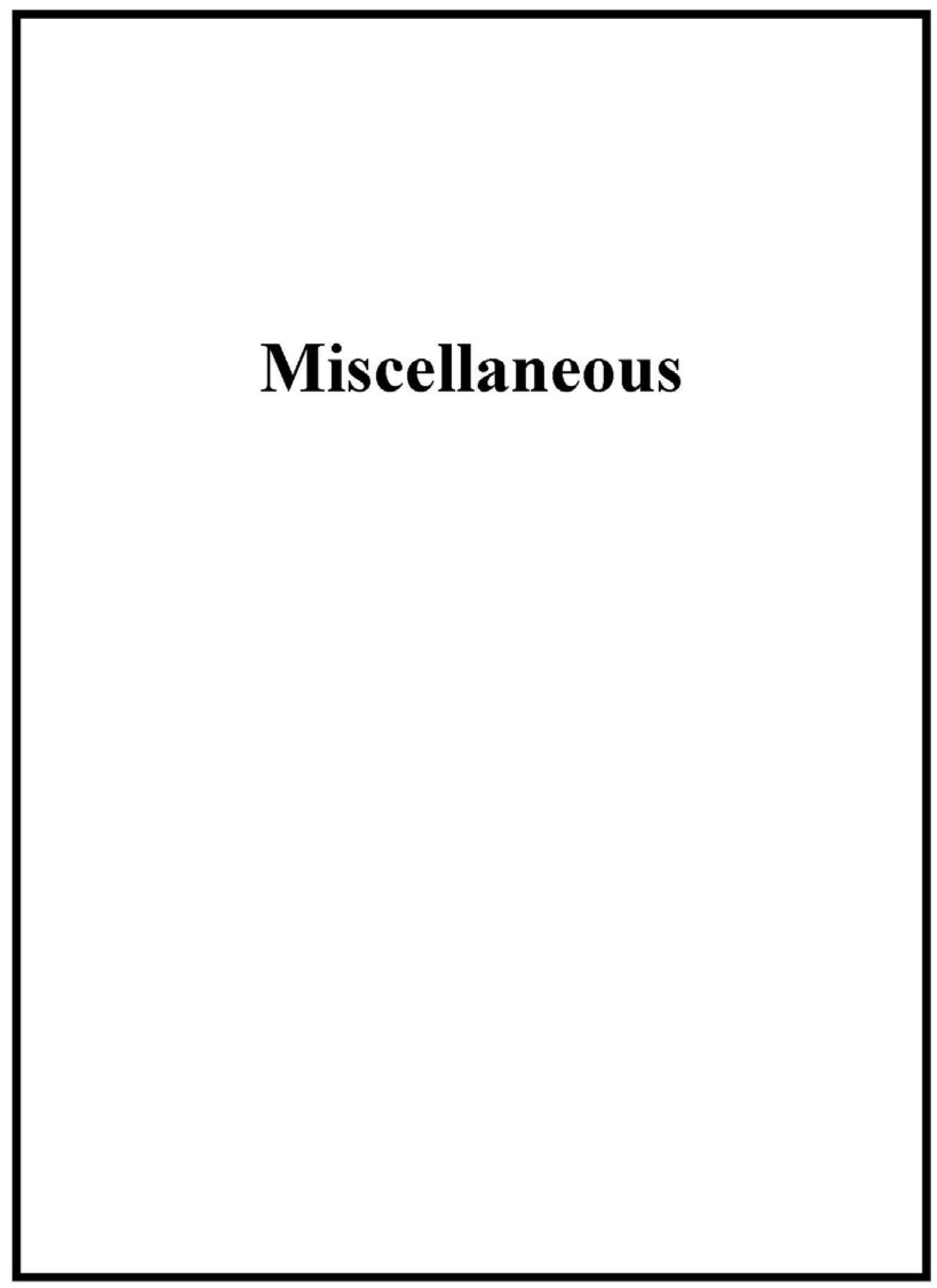

Page 47 of 94 


\section{Prep Logbook}

Closed-System Purge-and-Trap Collection and Extraction: Volatile Organics in Soil and Waste Samples

\begin{tabular}{|c|c|c|c|c|c|c|c|c|c|c|c|}
\hline Batch ID: & 175148 & & & Type & Sample Id & Description & & & Serial Number & Spike Amount & Spike Units \\
\hline Analyst: & Patrick & & & & & & & & & & \\
\hline Method: & SW846 & & & & & & & & & & \\
\hline Lab SOP: & GL-OA & 9 REV\# 12 & & & & & & & & & \\
\hline Instrument: & $\mathrm{OH} \mathrm{AL}$ & ance & & & & & & & & & \\
\hline Sample ID & & Prep Date & Matrix & $\begin{array}{l}\text { Tare Wt } \\
\text { (g) }\end{array}$ & $\begin{array}{l}\text { Final Wt } \\
\text { (g) }\end{array}$ & $\begin{array}{l}\text { Sample Wt } \\
(\mathrm{g})\end{array}$ & Preservative & $\begin{array}{l}\text { Final } \\
\text { Volume } \\
(\mathrm{mL})\end{array}$ & $\begin{array}{l}\text { Prep Factor } \\
(\mathrm{mL} / \mathrm{g})\end{array}$ & & \\
\hline 446517001 & & 22-MAR-2018 11:10:00 & Soil & 31.04 & 37.71 & 6.67 & DI WATER & 5 & 0.74963 & & \\
\hline 446517002 & & 22-MAR-201\$ 11:30:00 & Soil & 30.97 & 37.39 & 6.42 & DI WATER & 5 & 0.77882 & & \\
\hline 446517003 & & 22-MAR-2018 11:55:00 & Soil & 30.58 & 36.81 & 6.23 & DI WATER & 5 & 0.80257 & & \\
\hline 446517004 & & 22-MAR-2018 11:55:00 & Soil & 30.61 & 36.8 & 6.19 & DI WATER & 5 & 0.80775 & & \\
\hline $1203999288 \mathrm{MB}$ & & 29-MAR-2018 09:00:00 & Soil & & & 5 & DI WATER & 5 & 1 & & \\
\hline 1203999289 LCS & & 29-MAR-2018 09:01:00 & Soil & & & 5 & DI WATER & 5 & 1 & & \\
\hline 1203999290 LCSI & & 29-MAR-201 $809: 02: 00$ & Soil & & & 5 & DI WATER & 5 & 1 & & \\
\hline
\end{tabular}




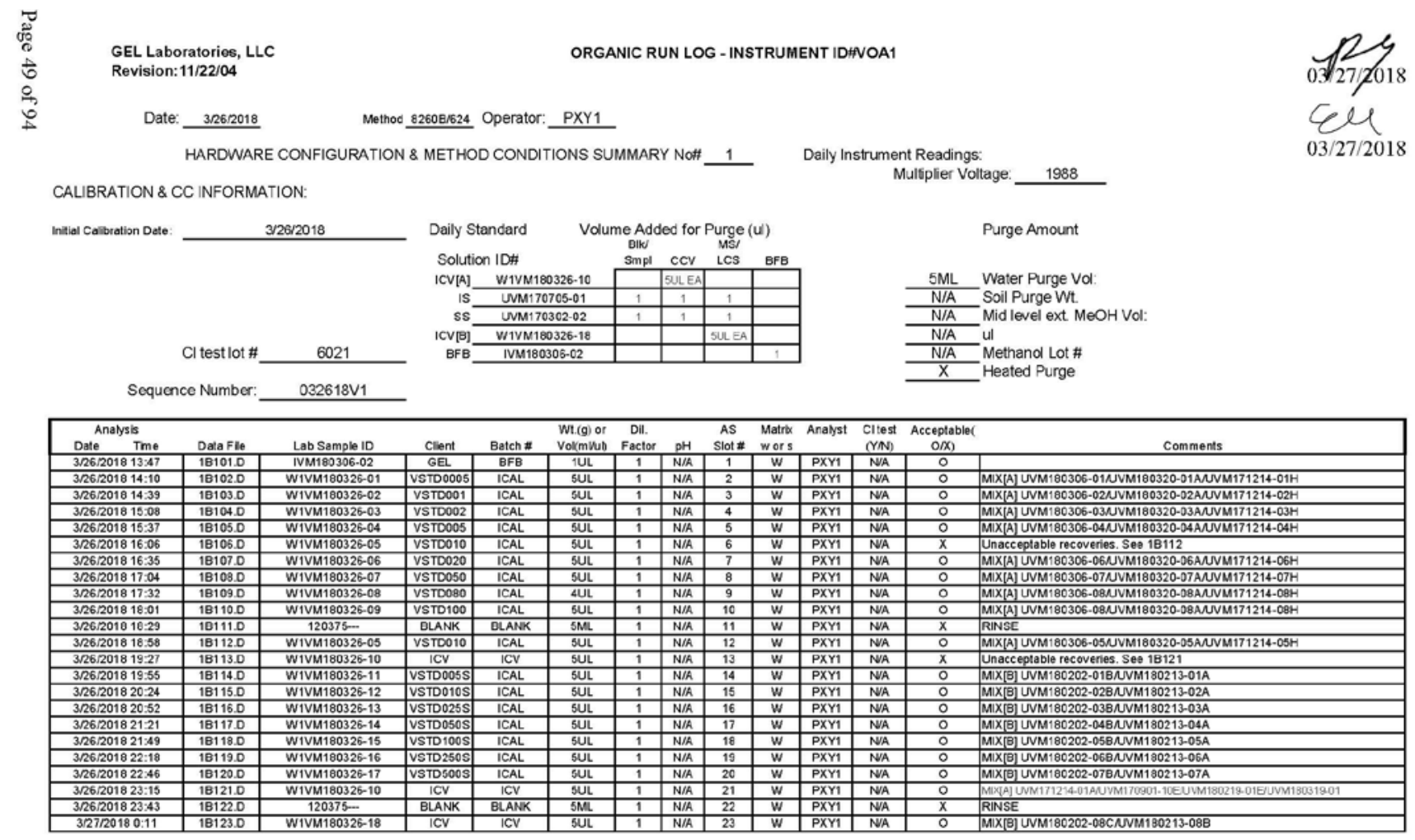

GL-OA-E-038, GL-OA-E-026, GL-OA-E-039 


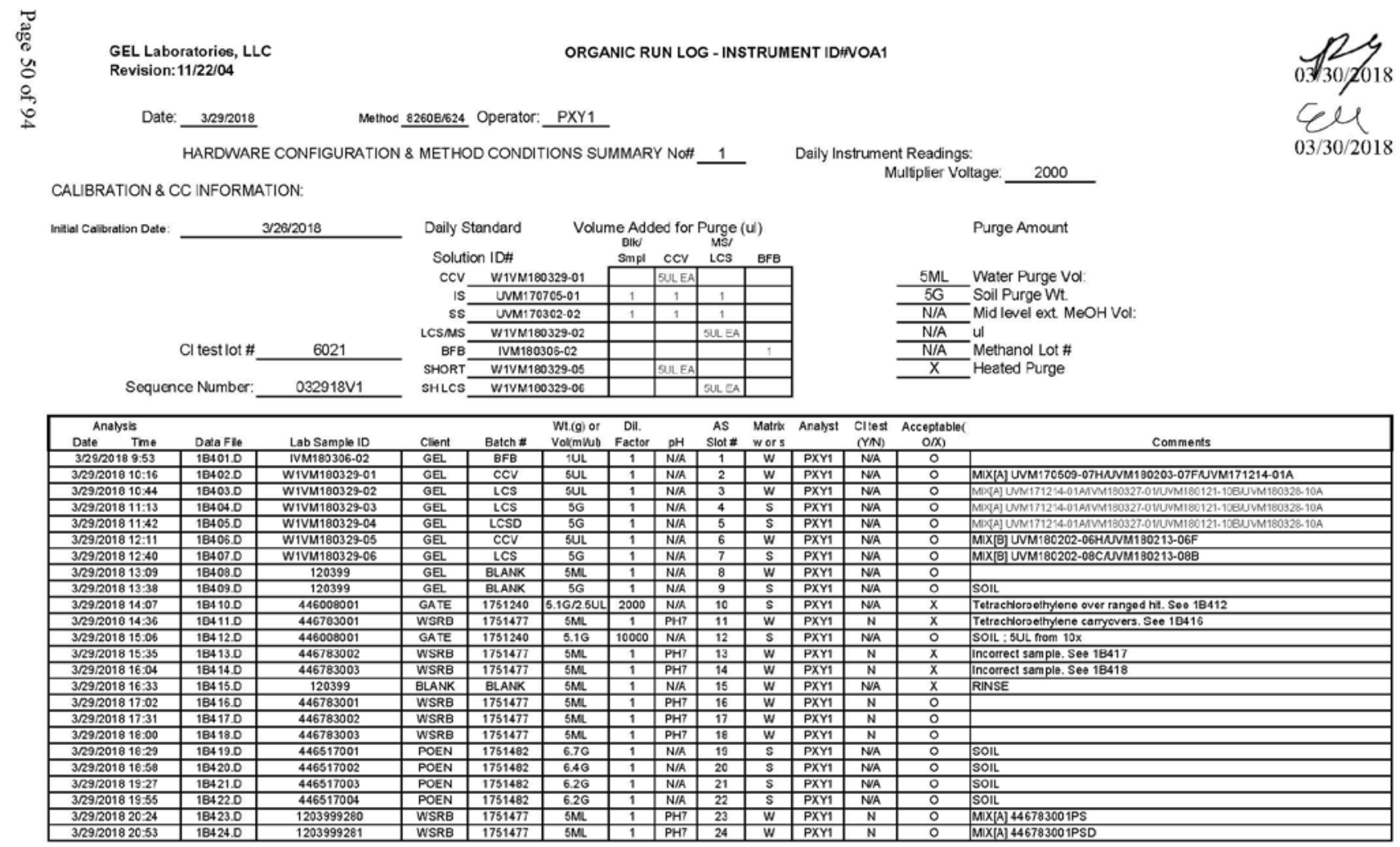

GL-OA-E-038, GL-OA-E-026, GL-OA-E-039 


\section{Semi-Volatile Analysis}

Page 51 of 94 


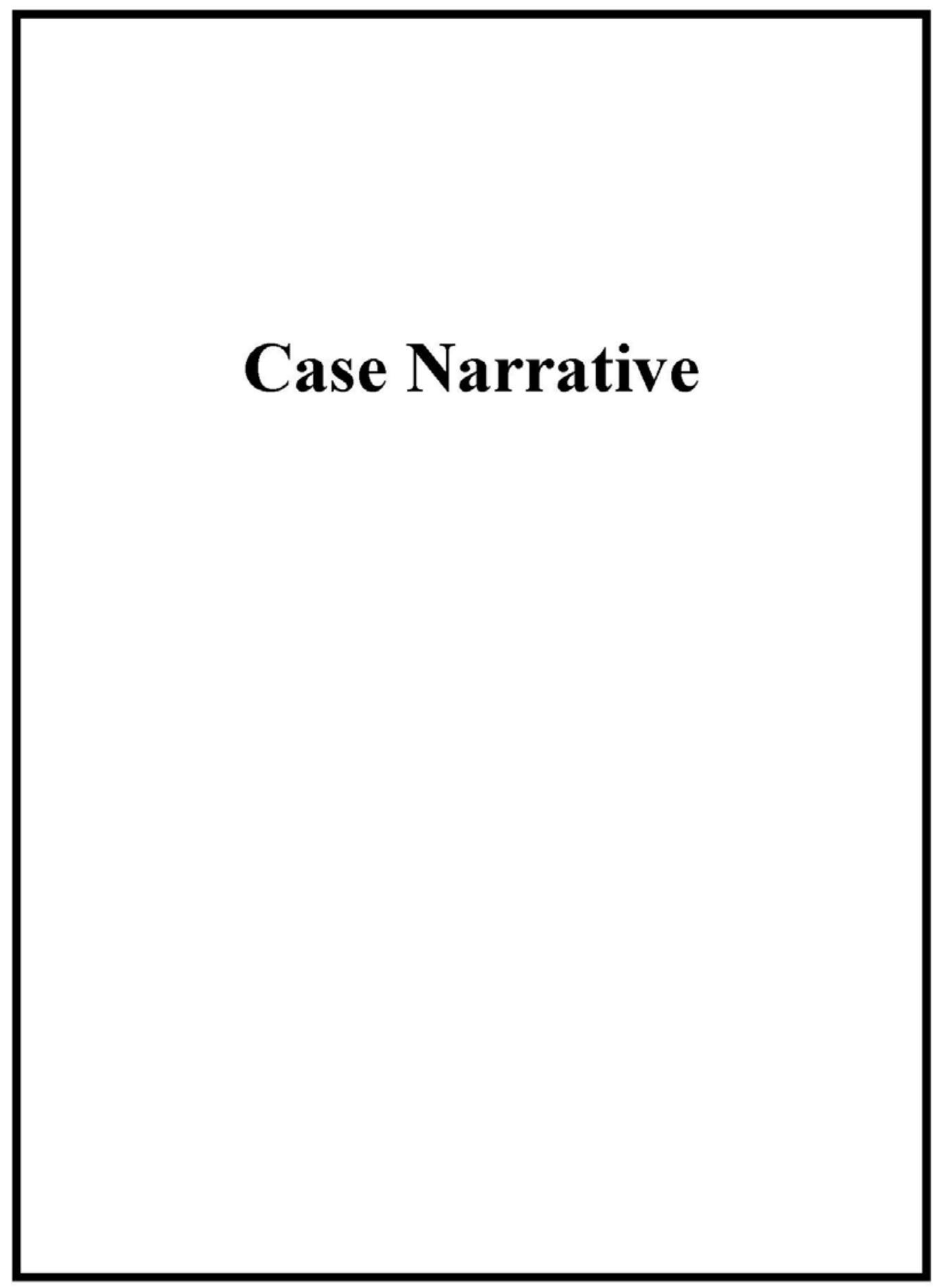

Page 52 of 94 


\title{
GC/MS Semivolatile \\ Technical Case Narrative \\ North Wind - Portage (POEN) \\ SDG \#: $\mathbf{4 4 6 5 1 7}$
}

\author{
Product: Analysis of Semivolatile Organic Compounds by Gas Chromatography/Mass Spectrometry \\ Analytical Method: SW846 3541/8270D SIM PAH \\ Analytical Procedure: GL-OA-E-009 REV\# 40 \\ Analytical Batch: 1750981 \\ Preparation Method: SW846 3541 \\ Preparation Procedure: GL-OA-E-066 REV\# 8 \\ Preparation Batch: 1750980
}

The following samples were analyzed using the above methods and analytical procedure(s).

\begin{tabular}{l} 
GEL Sample ID\# \\
\hline 446517001 \\
446517002 \\
446517003 \\
446517004 \\
1203998143 \\
1203998144 \\
1203998145 \\
1203998146
\end{tabular}

\author{
Client Sample Identification \\ CFA18001 - Soil Location \#1 \\ CFA18002 - Soil Location \#2 \\ CFA18003 - Soil Location \#3 \\ CFA18003 - DUP - Soil Location \#3 \\ Method Blank (MB) \\ Laboratory Control Sample (LCS) \\ 446517001(CFA18001 - Soil Location \#1) Matrix Spike (MS) \\ 446517001(CFA18001 - Soil Location \#1) Matrix Spike Duplicate (MSD)
}

The samples in this SDG were analyzed on a "dry weight" basis.

\section{Data Summary:}

All sample data provided in this report met the acceptance criteria specified in the analytical methods and procedures for initial calibration, continuing calibration, instrument controls and process controls where applicable, with the following exceptions.

\section{Quality Control (QC) Information}

MS/MSD Relative Percent Difference (RPD) Statement

The relative percent difference (RPD) between the MS and MSD (See Below) did not meet acceptance limits. As the individual MS and MSD recoveries were within the acceptance limits, the failures had no adverse impact on the reported sample data.

\begin{tabular}{|c|l|c|}
\hline Sample & Analyte & Value \\
\hline 1203998145MS and 1203998146MSD (CFA18001 - Soil Location \#1) & Pyrene & RPD 38* $(0 \%-30 \%)$ \\
\hline
\end{tabular}

\section{Technical Information}

Sample Re-extraction/Re-analysis

Sample 446517002 (CFA18002 - Soil Location \#2) was re-analyzed for ISTD failure.

\section{Certification Statement}

Page 53 of 94 
Where the analytical method has been performed under NELAP certification, the analysis has met all of the requirements of the NELAC standard unless otherwise noted in the analytical case narrative.

Page 54 of 94 


\author{
GEL LABORATORIES LLC \\ 2040 Savage Road Charleston SC 29407 - (843) 556-8171 - wuw.gel.com \\ Qualifier Definition Report \\ for \\ POEN004 N arth Wind - P ortage (8-00000013) \\ Client SDG: 446517 GEL Work Order: 446517
}

\title{
The Qualifiers in this report are defined as follows:
}

* A quality control analyte recovery is autside of specified acceptance criteria

** Analyte is a sunogate conqound

U Analyte was analyzed for, but not detected above the MDL, MDA, MDC or LOD.

DL Indicates that sample is dibuted.

RA Indicates that sample is re-analyzed without re-extraction.

RE Indicates that sample is re-extracted.

\section{Review/Vabidation}

GEL requires all analytical data to be verified by a qualified data reviewer. In addition, all CLP-like deliverbles receive a trird level review of the fractional data package.

The following data validator verified the irfomation presented in this data report:

signature: Bubane Banluy

Date: O4 APR 2018
Hame: Barbara Bailey

Title: Data Validator

Page 55 of 94 


\section{Sample Data Summary}

Page 56 of 94 


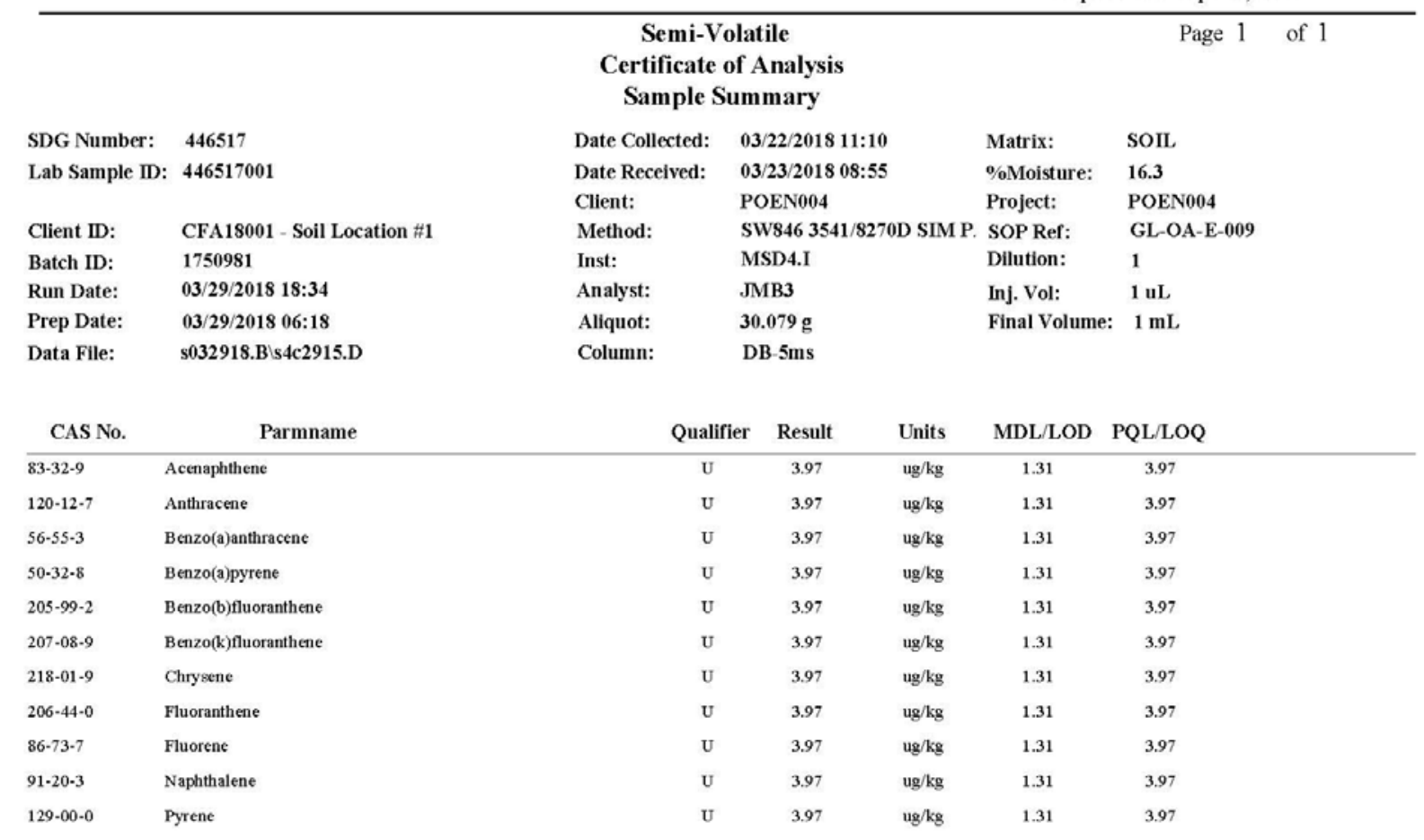

Page 57 of 94 


\begin{tabular}{|c|c|c|c|c|c|c|c|}
\hline \multirow{4}{*}{$\begin{array}{l}\text { SDG Number: } \\
\text { Lab Sample ID: }\end{array}$} & \multirow{4}{*}{$\begin{array}{l}446517 \\
446517002\end{array}$} & \multicolumn{4}{|c|}{$\begin{array}{c}\text { Semi-Volatile } \\
\text { Certificate of Analysis } \\
\text { Sample Summary }\end{array}$} & \multirow{4}{*}{$\begin{array}{l}\text { Matrix: } \\
\text { \%Moisture: } \\
\text { Project: }\end{array}$} & \multirow[t]{2}{*}{ Page 1 of 1} \\
\hline & & \multirow{3}{*}{$\begin{array}{l}\text { Date Collected: } \\
\text { Date Received: } \\
\text { Client: }\end{array}$} & \multicolumn{3}{|c|}{$03 / 22 / 201811: 30$} & & \\
\hline & & & \multicolumn{3}{|c|}{ 03/23/2018 08:55 } & & 21.8 \\
\hline & & & & & POEN004 \\
\hline Client ID: & CFA18002 - Soil Location $\# 2$ & Method: & \multirow{2}{*}{\multicolumn{3}{|c|}{$\begin{array}{l}\text { SW846 3541/8270D SIM P. } \\
\text { MSD4.I }\end{array}$}} & SOP Ref: & GL-OA-E-009 \\
\hline Batch ID: & 1750981 & Inst: & & & & Dilution: & 1 \\
\hline Run Date: & $03 / 30 / 2018$ 11:20 & Analyst: & \multicolumn{3}{|c|}{ JMB3 } & & $1 \mathrm{uL}$ \\
\hline Prep Date: & $03 / 29 / 201806: 18$ & Aliquot: & \multicolumn{3}{|c|}{$30.435 \mathrm{~g}$} & \multicolumn{2}{|c|}{ Final Volume: $1 \mathrm{~mL}$} \\
\hline Data File: & s033018.B \s4c3007.D & Column: & \multicolumn{3}{|c|}{ DB-5ms } & & \\
\hline CAS No. & Parmname & Quali & & Result & Units & MDL/LOD I & PQL/LOQ \\
\hline $83-32-9$ & Acenaphthene & $\mathrm{U}$ & & 4.20 & $\mathrm{ug} / \mathrm{kg}$ & 1.39 & 4.20 \\
\hline $120-12-7$ & Anthracene & $\mathrm{U}$ & & 4.20 & ug $/ \mathrm{kg}$ & 1.39 & 4.20 \\
\hline $56-55-3$ & Benzo(a)anthracene & $\mathrm{U}$ & & 4.20 & ug $/ \mathrm{kg}$ & 1.39 & 4.20 \\
\hline $50-32-8$ & Benzo(a)pyrene & $\mathrm{u}$ & & 4.20 & $\mathrm{ug} / \mathrm{kg}$ & 1.39 & 4.20 \\
\hline $205-99-2$ & Benzo(b)fluoranthene & $\mathrm{U}$ & & 4.20 & ug $/ \mathrm{kg}$ & 1.39 & 4.20 \\
\hline $207-08-9$ & Benzo(k)fluoranthene & $\mathrm{U}$ & & 4.20 & ug $/ \mathrm{kg}$ & 1.39 & 4.20 \\
\hline 218-01-9 & Chrysene & $\mathrm{U}$ & & 4.20 & $\mathrm{ug} / \mathrm{kg}$ & 1.39 & 4.20 \\
\hline $206-44-0$ & Fluoranthene & $\mathrm{U}$ & & 4.20 & ug $/ \mathrm{kg}$ & 1.39 & 4.20 \\
\hline $86-73-7$ & Fluorene & $\mathrm{U}$ & & 4.20 & ug $/ \mathrm{kg}$ & 1.39 & 4.20 \\
\hline $91-20-3$ & Naphthalene & $\mathrm{U}$ & & 4.20 & $\mathrm{ug} / \mathrm{kg}$ & 1.39 & 4.20 \\
\hline $129-00-0$ & Pyrene & $\mathrm{U}$ & & 4.20 & ug $/ \mathrm{kg}$ & 1.39 & 4.20 \\
\hline
\end{tabular}

Page 58 of 94 


\begin{tabular}{|c|c|c|c|c|c|c|c|}
\hline \multirow{4}{*}{$\begin{array}{l}\text { SDG Number: } \\
\text { Lab Sample ID: }\end{array}$} & \multirow{4}{*}{$\begin{array}{l}446517 \\
446517003\end{array}$} & \multicolumn{4}{|c|}{$\begin{array}{c}\text { Semi-Volatile } \\
\text { Certificate of Analysis } \\
\text { Sample Summary }\end{array}$} & \multirow{2}{*}{\multicolumn{2}{|c|}{ Page 1 of 1}} \\
\hline & & \multirow{3}{*}{$\begin{array}{l}\text { Date Collected: } \\
\text { Date Received: } \\
\text { Client: }\end{array}$} & \multicolumn{3}{|c|}{$03 / 22 / 201811: 55$} & & \\
\hline & & & \multirow{2}{*}{\multicolumn{3}{|c|}{$\begin{array}{l}\text { 03/23/2018 08:55 } \\
\text { POEN004 }\end{array}$}} & \%Moisture: & 6.8 \\
\hline & & & \multirow{3}{*}{\multicolumn{3}{|c|}{$\begin{array}{l}\text { POEN004 } \\
\text { SW846 3541/8270D SIM P. } \\
\text { MSD4.I }\end{array}$}} & Project: & POEN004 \\
\hline Client ID: & CFA18003 - Soil Location $\# 3$ & Method: & & & & SOP Ref: & GL-OA-E-009 \\
\hline Batch ID: & 1750981 & Inst: & & & & Dilution: & 1 \\
\hline Run Date: & $03 / 29 / 2018$ 19:31 & Analyst: & \multicolumn{3}{|c|}{ JMB3 } & Inj. Vol: & $1 \mathrm{uL}$ \\
\hline Prep Date: & 03/29/2018 06:18 & Aliquot: & \multicolumn{3}{|c|}{$30.124 \mathrm{~g}$} & \multicolumn{2}{|c|}{ Final Volume: $1 \mathrm{~mL}$} \\
\hline Data File: & s032918.B \s4c2917.D & Column: & & $5 \mathrm{~ms}$ & & & \\
\hline CAS No. & Parmname & Qualif & & Result & Units & $\mathrm{MDL} / \mathrm{LOD} \quad \mathrm{P}$ & PQL/LOQ \\
\hline $83-32-9$ & Acenaphthene & $\mathrm{U}$ & & 3.56 & $\mathrm{ug} / \mathrm{kg}$ & 1.18 & 3.56 \\
\hline $120-12-7$ & Anthracene & $\mathrm{U}$ & & 3.56 & ug/kg & 1.18 & 3.56 \\
\hline $56-55-3$ & Benzo(a)anthracene & $\mathrm{U}$ & & 3.56 & $\mathrm{ug} / \mathrm{kg}$ & 1.18 & 3.56 \\
\hline $50-32-8$ & Benzo(a)pyrene & $\mathrm{U}$ & & 3.56 & $\mathrm{ug} / \mathrm{kg}$ & 1.18 & 3.56 \\
\hline $205-99-2$ & Benzo(b)fluoranthene & $\mathrm{U}$ & & 3.56 & $\mathrm{ug} / \mathrm{kg}$ & 1.18 & 3.56 \\
\hline $207-08-9$ & Benzo(k)fluoranthene & $\mathrm{U}$ & & 3.56 & $\mathrm{ug} / \mathrm{kg}$ & 1.18 & 3.56 \\
\hline 218-01-9 & Chrysene & $\mathrm{U}$ & & 3.56 & $\mathrm{ug} / \mathrm{kg}$ & 1.18 & 3.56 \\
\hline $206-44-0$ & Fluoranthene & $\mathrm{U}$ & & 3.56 & $\mathrm{ug} / \mathrm{kg}$ & 1.18 & 3.56 \\
\hline $86-73-7$ & Fluorene & $\mathrm{U}$ & & 3.56 & $\mathrm{ug} / \mathrm{kg}$ & 1.18 & 3.56 \\
\hline $91-20-3$ & Naphthalene & $\mathrm{U}$ & & 3.56 & $\mathrm{ug} / \mathrm{kg}$ & 1.18 & 3.56 \\
\hline $129-00-0$ & Pyrene & $\mathrm{U}$ & & 3.56 & $\mathrm{ug} / \mathrm{kg}$ & 1.18 & 3.56 \\
\hline
\end{tabular}

Page 59 of 94 


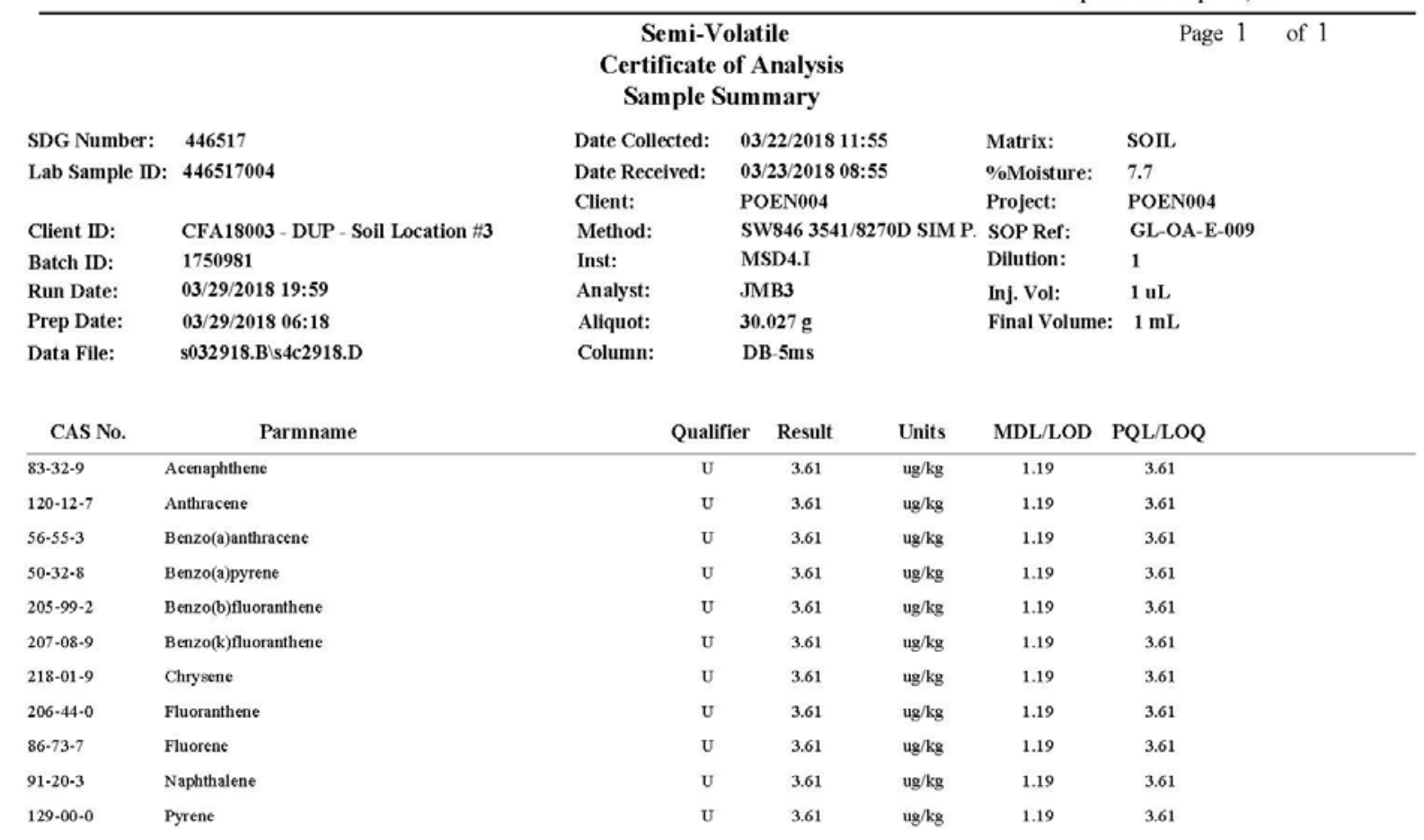




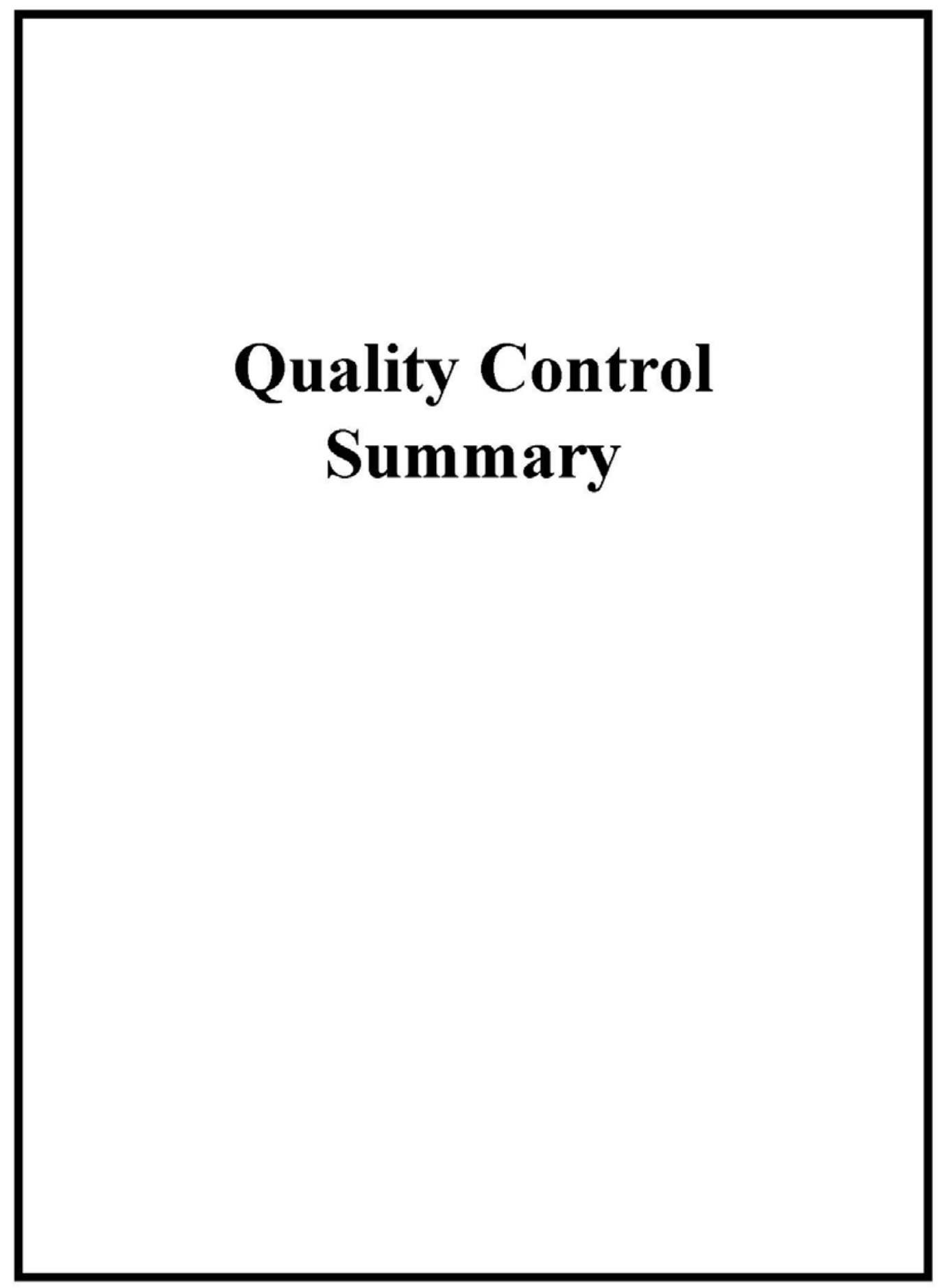

Page 61 of 94 


\section{Semi-Volatile \\ Surrogate Recovery Report}

Page 1

of 1

SDG Number: 446517

Matrix Type: SOLID

\begin{tabular}{llc}
\hline Sample ID & Client ID & $\begin{array}{c}\text { 5-alpha } \\
\text { \%REC }\end{array}$ \\
\hline 1203998144 & LCS for batch 1750980 & 80 \\
1203998145 & CFA18001 - Soil Location \#1MS & 73 \\
1203998146 & CFA18001 - Soil Location & 59 \\
1203998143 & \#1(446517001MSD & 85 \\
446517001 & CFA18001 - Soil Location \#1 & 68 \\
446517003 & CFA18003 - Soil Location \#3 & 75 \\
446517004 & CFA18003 - DUP - Soil Location \#3 & 68 \\
446517002 & CFA18002 - Soil Location \#2 & 64 \\
& & Acceptance Limits \\
Surrogate & & $(30 \%-118 \%)$ \\
5-alpha- $=$ 5-alpha-Androstane & \\
* Recovery outside Acceptance Limits & \\
\# Column to be used to flag recovery values & \\
D Sample Diluted &
\end{tabular}

Page 62 of 94 


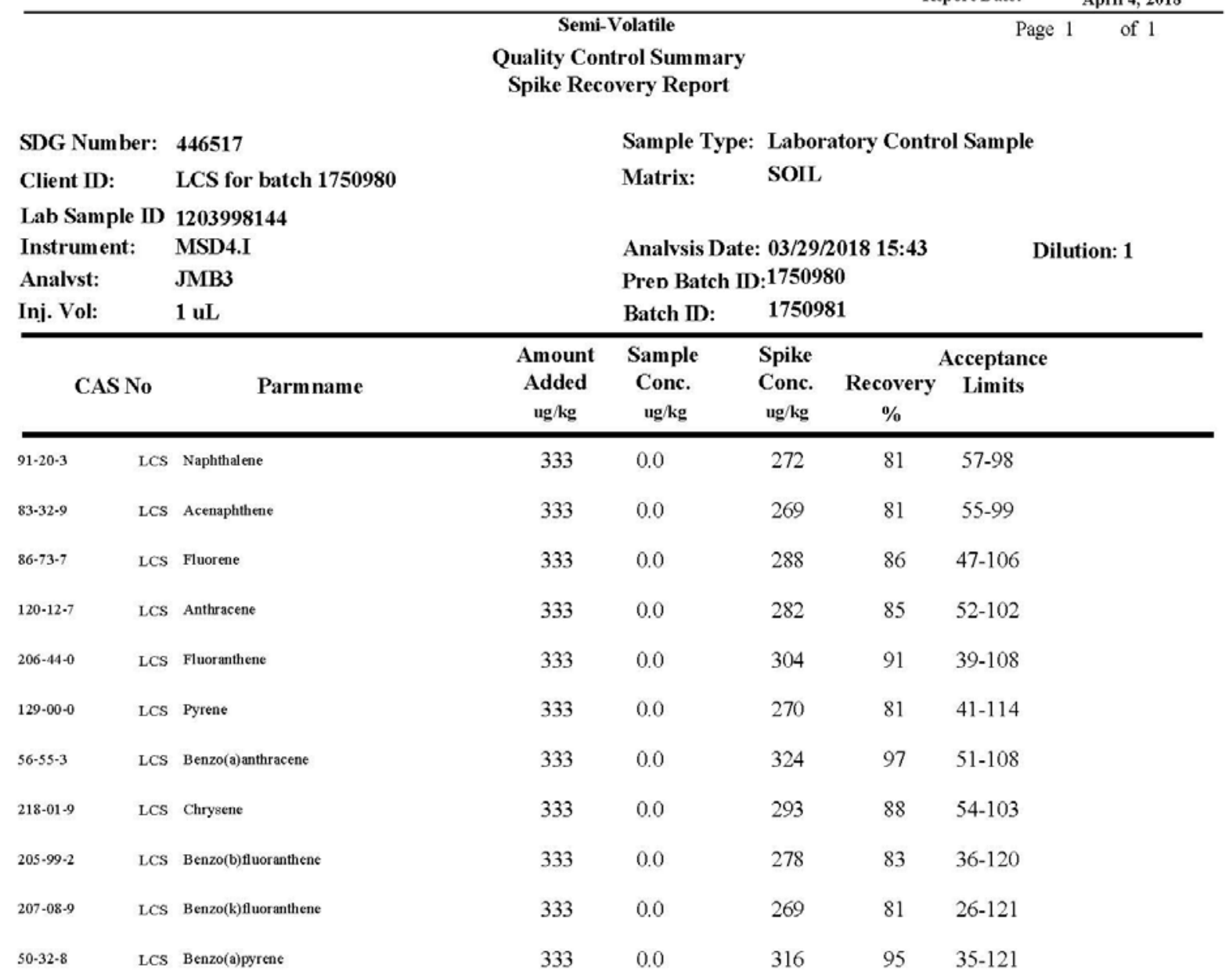




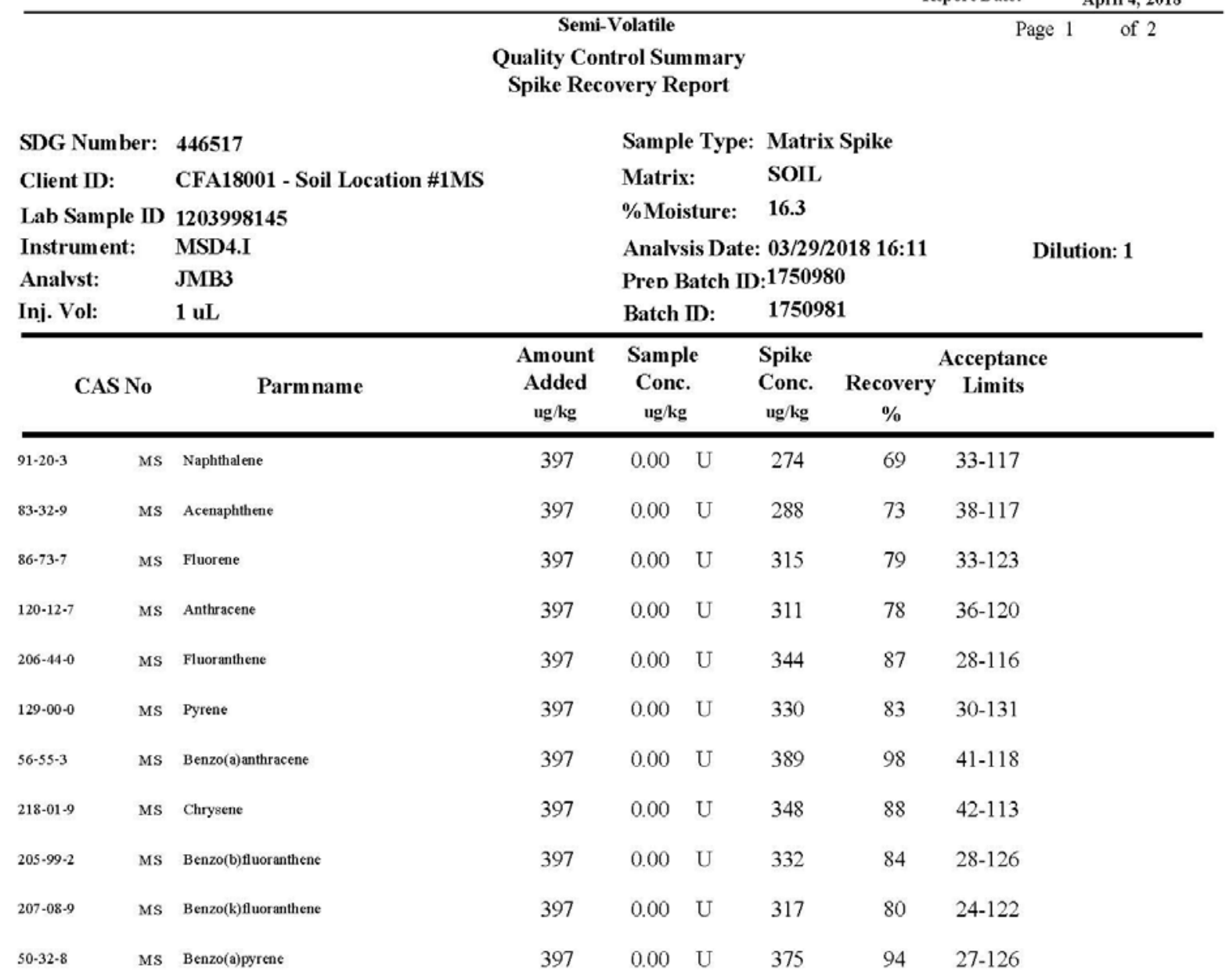




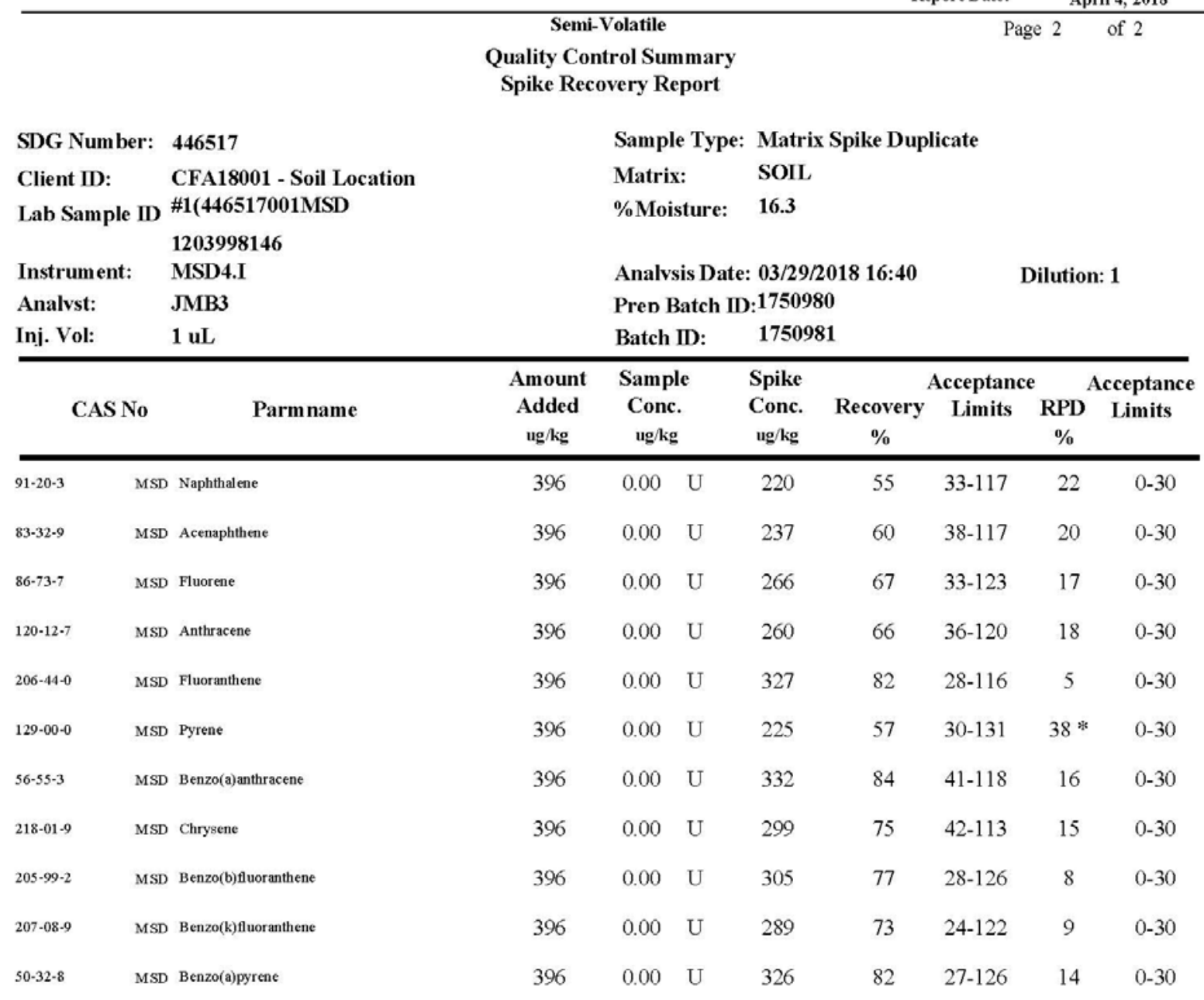




\begin{tabular}{|c|c|c|c|c|c|}
\hline \multirow{5}{*}{$\begin{array}{l}\text { SDG Number: } \\
\text { Client ID: } \\
\text { Lab Sample ID: } \\
\text { Column: }\end{array}$} & & \multicolumn{2}{|c|}{ Method Blank Summary } & \multirow{4}{*}{$\begin{array}{l}\text { Matrix: } \\
\text { Data File: } \\
\text { Analyzed: }\end{array}$} & Page 1 of 1 \\
\hline & & Client: & POEN004 & & SOIL \\
\hline & MB for batch 1750980 & Instrument ID: & MSD4.I & & s032918.B $\backslash 44 c 2913 . D$ \\
\hline & 1203998143 & Prep Date: & 03/29/2018 06:18 & & $03 / 29 / 1817: 37$ \\
\hline & DB-5ms & & & & \\
\hline \multicolumn{6}{|c|}{ This method blank applies to the following samples and quality control samples: } \\
\hline \multicolumn{2}{|c|}{ Client Sample ID } & Lab Sample ID & File ID & Date Analyzed & \multirow{2}{*}{$\begin{array}{c}\text { Time Analyzed } \\
1543\end{array}$} \\
\hline 01 & LCS for batch 1750980 & 1203998144 & s032918.B $\backslash \mathrm{s} 4 \mathrm{c} 2909 . \mathrm{D}$ & & \\
\hline 02 & CFA18001 - Soil Location $\# 1 M S$ & 1203998145 & s032918.B $\backslash \mathrm{s} 4 \mathrm{c} 2910 . \mathrm{D}$ & $03 / 29 / 18$ & 1611 \\
\hline 03 & $\begin{array}{l}\text { CFA18001 - Soil Location } \\
\# 1(446517001 \mathrm{MSD}\end{array}$ & 1203998146 & s032918.B $\backslash$ s4c2911.D & $03 / 29 / 18$ & 1640 \\
\hline 04 & CFA18001 - Soil Location \#1 & 446517001 & s032918.B $1 \mathrm{~s} 4 \mathrm{c} 2915 . \mathrm{D}$ & $03 / 29 / 18$ & 1834 \\
\hline 05 & CFA18003 - Soil Location $\# 3$ & 446517003 & s032918.B 1 s4c2917.D & $03 / 29 / 18$ & 1931 \\
\hline 06 & CFA18003 - DUP - Soil Location $\# 3$ & 446517004 & s032918.B $\backslash$ s4c2918.D & $03 / 29 / 18$ & 1959 \\
\hline 07 & CFA18002 - Soil Location $\# 2$ & 446517002 & s033018.B $\backslash s 4 c 3007 . D$ & $03 / 30 / 18$ & 1120 \\
\hline
\end{tabular}




\section{Instrument Performance Check}

DFTPP

Lab Name GEL Laboratories LLC

Instrument ID: MSD4.I

Column Description: DB-5ms
Client SDG: 446517

Injection Date/Time: 01-MAR-18 09:09

\begin{tabular}{|c|c|c|}
\hline $\mathbf{m} / \mathbf{e}$ & Ion Abundance Criteria & \% Relative Abundance \\
\hline 51 & $10-80 \%$ of mass 198 & 44.5 \\
\hline 68 & Less than $2 \%$ of mass 69 & 1.4 \\
\hline 69 & Mass 69 Relative Abundance & 43.9 \\
\hline 70 & Less than $2 \%$ of mass 69 & 0.5 \\
\hline 127 & $10-80 \%$ of mass 198 & 53.2 \\
\hline 197 & Less than $2 \%$ of mass 198 & 0 \\
\hline 198 & Base Peak, $100 \%$ Relative Abundance & 100 \\
\hline 199 & $5-9 \%$ of mass 198 & 6.8 \\
\hline 275 & $10-60 \%$ of mass 198 & 24.6 \\
\hline 365 & Greater than $1 \%$ of mass 198 & 3.4 \\
\hline 441 & Less than $24 \%$ of mass 442 & 15.7 \\
\hline 442 & Greater than $50 \%$ of mass 198 & 85.3 \\
\hline 443 & $15-24 \%$ of mass 442 & 19.5 \\
\hline
\end{tabular}

THIS CHECK APPLIES TO THE FOLLOWING SAMPLES, MS, MSD, LCS, LCSD,BLANKS AND STANDARDS

\begin{tabular}{|c|c|c|c|}
\hline $\begin{array}{c}\text { Client } \\
\text { Sample ID }\end{array}$ & $\begin{array}{c}\text { Lab } \\
\text { Sample ID }\end{array}$ & $\begin{array}{c}\text { Lab } \\
\text { File ID }\end{array}$ & $\begin{array}{c}\text { Time } \\
\text { Analyzed }\end{array}$ \\
\hline ICALMIX[A,B] & WBN180209-88 & s030118.B\s4c0102.D & 01-MAR-18 09:25 \\
\hline ICALMIX[A,B] & WBN180209-86 & s030118.B \s4c0103.D & 01-MAR-18 09:53 \\
\hline ICALMIX[A,B] & WBN180209-85 & s030118.B\s4c0104.D & 01-MAR-18 10:21 \\
\hline ICALMIX[A,B] & WBN180209-83 & s030118.B\s4c0105.D & 01-MAR-18 10:49 \\
\hline ICALMIX[A,B] & WBN180209-82 & s030118.B \s4c0106.D & 01-MAR-18 11:17 \\
\hline ICALMIX[A,B] & WBN180209-81 & s030118.B \s4c0107.D & 01-MAR-18 11:46 \\
\hline ICVMSDS417D_S01 & WBN180301-89 & s030118.B\s4c0108.D & 01-MAR-18 12:16 \\
\hline
\end{tabular}




\section{Instrument Performance Check}

DFTPP

Lab Name GEL Laboratories LLC

Instrument ID: MSD4.I

Column Description: DB-5ms
Client SDG: 446517

Injection Date/Time: 29-MAR-18 14:56

\begin{tabular}{|c|c|c|}
\hline $\mathbf{m} / \mathbf{e}$ & Ion Abundance Criteria & \% Relative Abundance \\
\hline 51 & $10-80 \%$ of mass 198 & 46.4 \\
\hline 68 & Less than $2 \%$ of mass 69 & 1.4 \\
\hline 69 & Mass 69 Relative Abundance & 43.8 \\
\hline 70 & Less than $2 \%$ of mass 69 & 0.5 \\
\hline 127 & $10-80 \%$ of mass 198 & 52.6 \\
\hline 197 & Less than $2 \%$ of mass 198 & 0.8 \\
\hline 198 & Base Peak, $100 \%$ Relative Abundance & 100 \\
\hline 199 & $5-9 \%$ of mass 198 & 6.7 \\
\hline 275 & $10-60 \%$ of mass 198 & 24.1 \\
\hline 365 & Greater than $1 \%$ of mass 198 & 3.4 \\
\hline 441 & Less than $24 \%$ of mass 442 & 15.4 \\
\hline 442 & Greater than $50 \%$ of mass 198 & 83.8 \\
\hline 443 & $15-24 \%$ of mass 442 & 19.2 \\
\hline
\end{tabular}

THIS CHECK APPLIES TO THE FOLLOWING SAMPLES, MS, MSD, LCS, LCSD,BLANKS AND STANDARDS

\begin{tabular}{|l|l|l|l|}
\hline \multicolumn{1}{|c|}{$\begin{array}{c}\text { Client } \\
\text { Sample ID }\end{array}$} & \multicolumn{1}{c|}{$\begin{array}{c}\text { Lab } \\
\text { Sample ID }\end{array}$} & \multicolumn{1}{c|}{$\begin{array}{c}\text { Lab } \\
\text { File ID }\end{array}$} & $\begin{array}{c}\text { Time } \\
\text { Analyzed }\end{array}$ \\
\hline CCVMLX[A]01 & WBN180311-86.3 & $s 032918 . B \backslash s 4 c 2908 . D$ & $29-M A R-1815: 12$ \\
\hline BLK01LCS & 1203998144 & $s 032918 . B \backslash s 4 c 2909 . D$ & $29-M A R-1815: 43$ \\
\hline CFA18001 - Soil Location \#1MS & 1203998145 & $s 032918 . B \backslash s 4 c 2910 . D$ & $29-M A R-1816: 11$ \\
\hline CFA18001 - Soil Location \#1MS & 1203998146 & $s 032918 . B \backslash s 4 c 2911 . D$ & $29-M A R-1816: 40$ \\
\hline BLK01 & 1203998143 & $s 032918 . B \backslash s 4 c 2913 . D$ & $29-M A R-1817: 37$ \\
\hline CFA18001 - Soil Location \#1 & 446517001 & $s 032918 . B \backslash s 4 c 2915 . D$ & $29-M A R-1818: 34$ \\
\hline CFA18003 - Soil Location \#3 & 446517003 & $s 032918 . B \backslash s 4 c 2917 . D$ & $29-M A R-1819: 31$ \\
\hline CFA18003 - DUP - Soil Location & 446517004 & $s 032918 . B \backslash s 4 c 2918 . D$ & $29-M A R-1819: 59$ \\
\hline
\end{tabular}




\section{Instrument Performance Check}

DFTPP

Lab Name GEL Laboratories LLC

Instrument ID: MSD4.I

Column Description: DB-5ms
Client SDG: 446517

Injection Date/Time: 30-MAR-18 10:04

Lab File ID s033018.Bis4c3004.D

\begin{tabular}{|c|c|c|}
\hline $\mathbf{m} / \mathbf{e}$ & Ion Abundance Criteria & \% Relative Abundance \\
\hline 51 & $10-80 \%$ of mass 198 & 46.8 \\
\hline 68 & Less than $2 \%$ of mass 69 & 1.6 \\
\hline 69 & Mass 69 Relative Abundance & 44.2 \\
\hline 70 & Less than $2 \%$ of mass 69 & 0.5 \\
\hline 127 & $10-80 \%$ of mass 198 & 52.4 \\
\hline 197 & Less than $2 \%$ of mass 198 & 0.9 \\
\hline 198 & Base Peak, $100 \%$ Relative Abundance & 100 \\
\hline 199 & $5-9 \%$ of mass 198 & 6.9 \\
\hline 275 & $10-60 \%$ of mass 198 & 23.5 \\
\hline 365 & Greater than $1 \%$ of mass 198 & 3.4 \\
\hline 441 & Less than $24 \%$ of mass 442 & 15.5 \\
\hline 442 & Greater than $50 \%$ of mass 198 & 78 \\
\hline 443 & $15-24 \%$ of mass 442 & 19.4 \\
\hline
\end{tabular}

THIS CHECK APPLIES TO THE FOLLOWING SAMPLES, MS, MSD, LCS, LCSD,BLANKS AND STANDARDS

\begin{tabular}{|c|c|c|c|}
\hline $\begin{array}{c}\text { Client } \\
\text { Sample ID }\end{array}$ & $\begin{array}{c}\text { Lab } \\
\text { Sample ID }\end{array}$ & $\begin{array}{c}\text { Lab } \\
\text { File ID }\end{array}$ & $\begin{array}{c}\text { Time } \\
\text { Analyzed }\end{array}$ \\
\hline CCVMIX[A]02 & WBN180311-86.3 & s033018.B\s4c3005.D & 30-MAR-18 10:20 \\
\hline CFA18002 - Soil Location \#2 & 446517002 & s033018.B \s4c3007.D & 30-MAR-18 11:20 \\
\hline
\end{tabular}




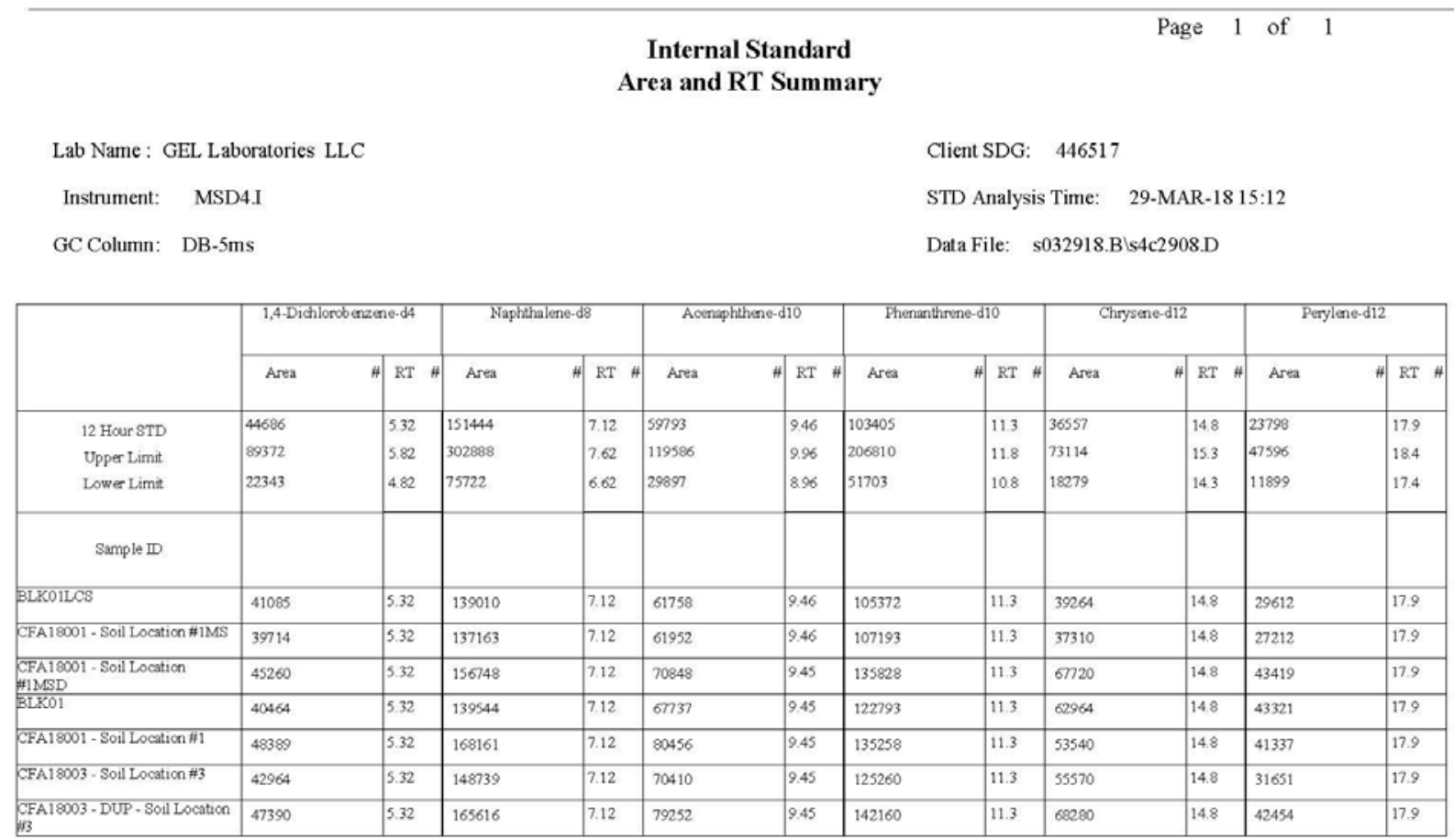

Area Upper Limit $=+100 \%$ of internal standard area

Area Lower Limit $=-50 \%$ of intemal standard area

RT Upper Limit $=+0.50$ minutes of internal standard RT

RT Lower Limit $=-0.50$ minutes of internal standard RT

\# Column used to flag values outside QC limits with an asterisk

" Value outside of QC Limits

Page 70 of 94 


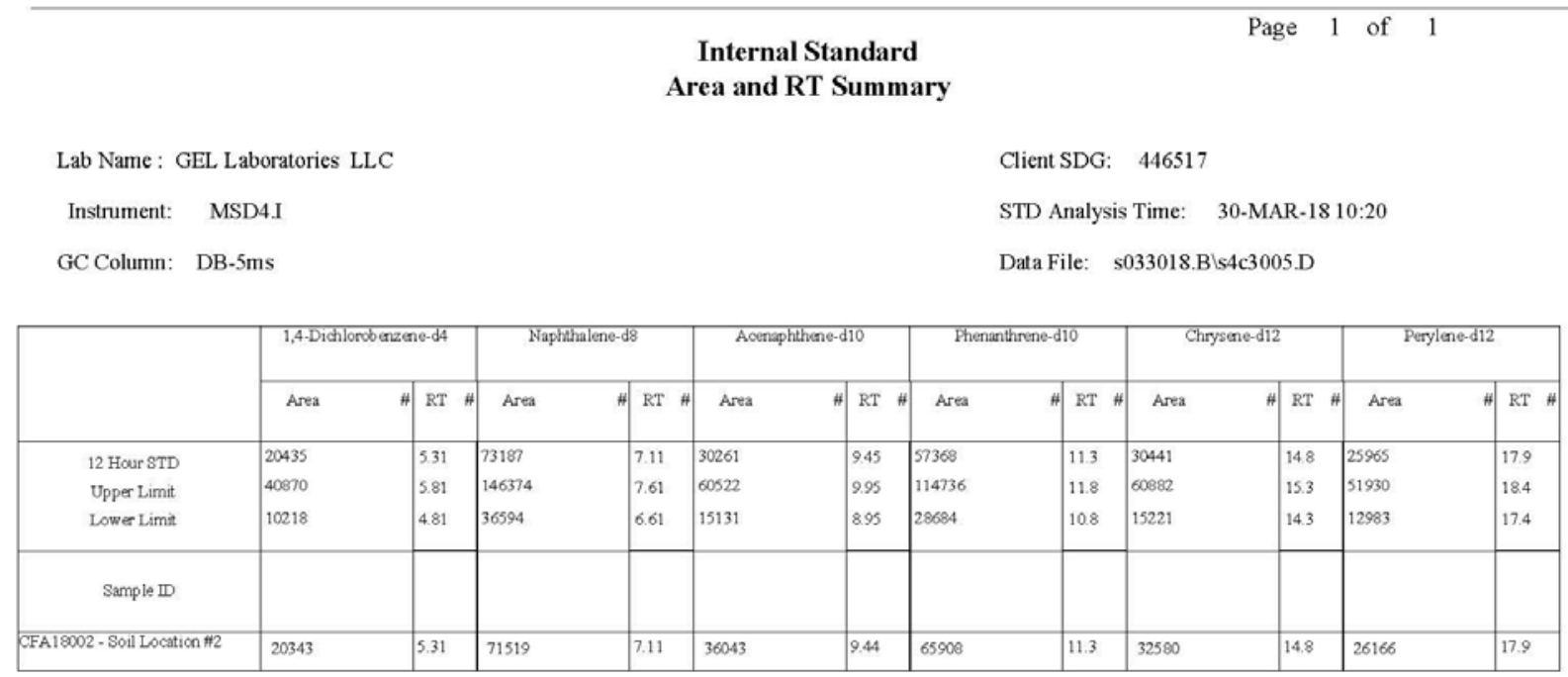

Area Upper Limit $=+100 \%$ of internal standard area

Area Lower Limit $=-50 \%$ of internal standard area

RT Upper Limit $=+0.50$ minutes of internal standard RT

RT Lower Limit $=-0.50$ minutes of internal standard RT

\# Column used to flag values outside QC limits with an asterisk

" Value outside of QC Limits

Page 71 of 94 


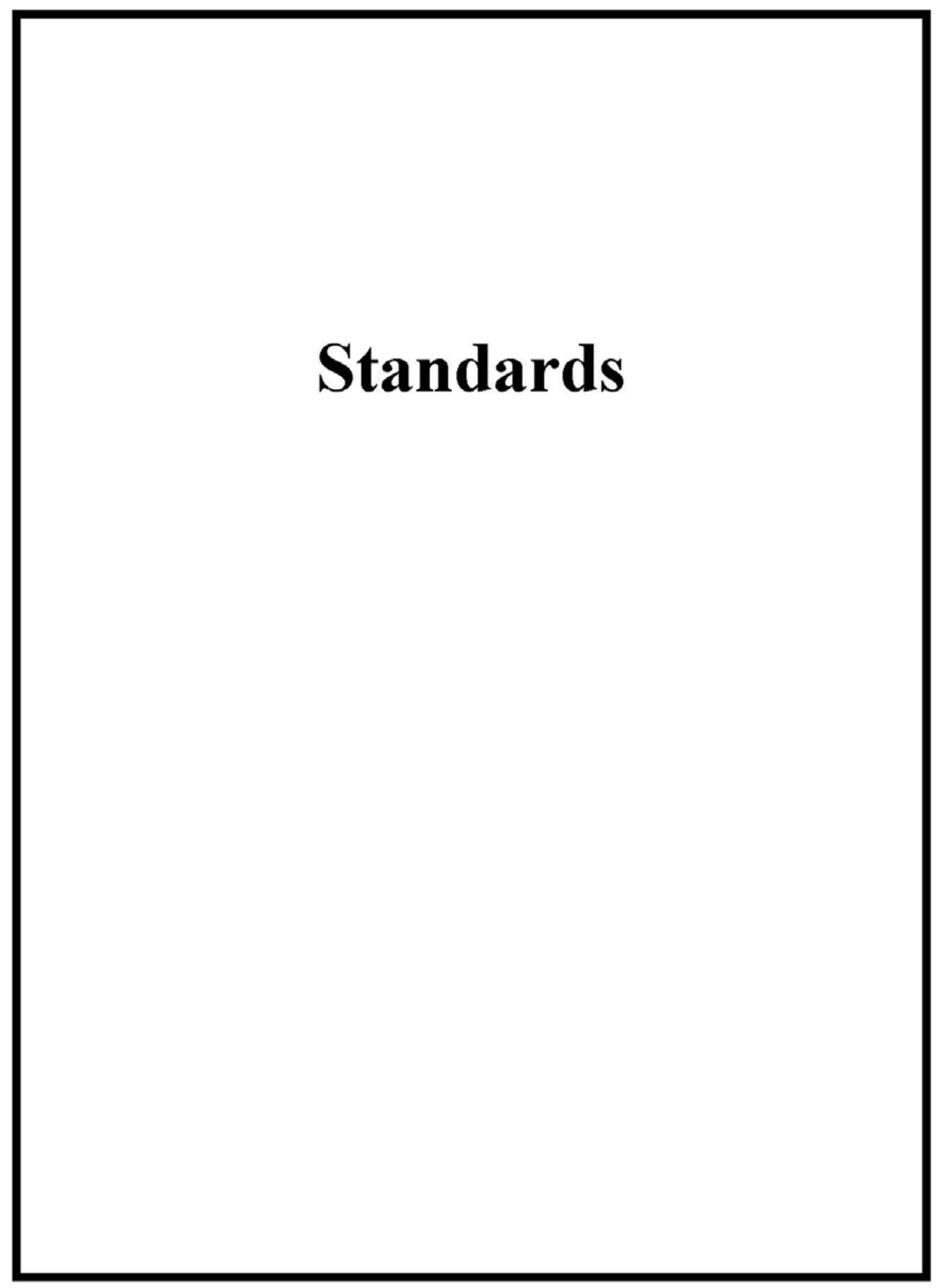

Page 72 of 94 


\begin{tabular}{|c|c|c|c|c|c|c|c|c|c|c|}
\hline SW846 8270/EPA 625 & & & & & & & & & & \\
\hline Calibration Standard Concentration Levels ${ }^{\star}$ & & & & & & & & & & \\
\hline MEGA MIX & Level 1 & Level 2 & Level3 & Level 4f & Level 5 & Level 6 & Level 7 & Level 8 & Level 9 & Level 10 \\
\hline 1,4-Dichlorobenzene-d4 (INTERNAL STANDARD & & & & & & & & & & \\
\hline Naphthalene-d8 (INTERNAL STANDARD) & & & & & & & & & & \\
\hline Acenaphthene-d10 (INTERNAL STANDARD) & & & & & & & & & & \\
\hline Phenanthrene-d10 (INTERNAL STANDARD) & & & & & & & & & & \\
\hline Chrysene-d12 (INTERNAL STANDARD) & & & & & & & & & & \\
\hline Perylene-d12 (INTERNAL STANDARD) & & & & & & & & & & \\
\hline 2-Fluorophenol (SURROGATE) & & 10 & 20 & 40 & 50 & 80 & 100 & 120 & 30 & 60 \\
\hline Phenol-d5 (SURROGATE) & & 10 & 20 & 40 & 50 & 80 & 100 & 120 & 30 & 60 \\
\hline 2-Chlorophenol-d4 (CLP SURROGATE) & & 10 & 20 & 40 & 50 & 80 & 100 & 120 & 30 & 60 \\
\hline 1,2-Dichlorobenzene-d4 (CLP SURROGATE) & & 10 & 20 & 40 & 50 & 80 & 100 & 120 & 30 & 60 \\
\hline Nitrobenzene-d5 (SURROGATE) & & 10 & 20 & 40 & 50 & 80 & 100 & 120 & 30 & 60 \\
\hline 2-Fluorobiphenyl (SURROGATE) & & 10 & 20 & 40 & 50 & 80 & 100 & 120 & 30 & 60 \\
\hline 2,4,6-Tribromophenol (SURROGATE) & & 10 & 20 & 40 & 50 & 80 & 100 & 120 & 30 & 60 \\
\hline p-Terphenyl-d14 (SURROGATE) & & 10 & 20 & 40 & 50 & 80 & 100 & 120 & 30 & 60 \\
\hline N-Nitrosodimethylamine & $1^{\star \star}$ & 10 & 20 & 40 & 50 & 80 & 100 & 120 & 30 & 60 \\
\hline Pyridine & & 10 & 20 & 40 & 50 & 80 & 100 & 120 & 30 & 60 \\
\hline Aniline & & 10 & 20 & 40 & 50 & 80 & 100 & 120 & 30 & 60 \\
\hline Phenol & & 10 & 20 & 40 & 50 & 80 & 100 & 120 & 30 & 60 \\
\hline bis(2-Chloroethyl)ether & & 10 & 20 & 40 & 50 & 80 & 100 & 120 & 30 & 60 \\
\hline 2-Chlorophenol & & 10 & 20 & 40 & 50 & 80 & 100 & 120 & 30 & 60 \\
\hline n-Decane & & 10 & 20 & 40 & 50 & 80 & 100 & 120 & 30 & 60 \\
\hline 1,3-Dichlorobenzene & & 10 & 20 & 40 & 50 & 80 & 100 & 120 & 30 & 60 \\
\hline 1,4-Dichlorobenzene & & 10 & 20 & 40 & 50 & 80 & 100 & 120 & 30 & 60 \\
\hline Benzyl Alcohol & & 10 & 20 & 40 & 50 & 80 & 100 & 120 & 30 & 60 \\
\hline 1,2-Dichlorobenzene & & 10 & 20 & 40 & 50 & 80 & 100 & 120 & 30 & 60 \\
\hline bis(2-Chloro-1-methylethyl)ether & & 10 & 20 & 40 & 50 & 80 & 100 & 120 & 30 & 60 \\
\hline o-Cresol (2-Methylphenol) & & 10 & 20 & 40 & 50 & 80 & 100 & 120 & 30 & 60 \\
\hline N-Nitrosodipropylamine & & 10 & 20 & 40 & 50 & 80 & 100 & 120 & 30 & 60 \\
\hline m,p-Cresols (3-Methylphenol \& 4-Methylphenol) & & 10 & 20 & 40 & 50 & 80 & 100 & 120 & 30 & 60 \\
\hline Hexachloroethane & & 10 & 20 & 40 & 50 & 80 & 100 & 120 & 30 & 60 \\
\hline Nitrobenzene & & 10 & 20 & 40 & 50 & 80 & 100 & 120 & 30 & 60 \\
\hline Isophorone & & 10 & 20 & 40 & 50 & 80 & 100 & 120 & 30 & 60 \\
\hline 2-Nitrophenol & & 10 & 20 & 40 & 50 & 80 & 100 & 120 & 30 & 60 \\
\hline 2,4-Dimethylphenol & & 10 & 20 & 40 & 50 & 80 & 100 & 120 & 30 & 60 \\
\hline bis(2-Chloroethoxy)methane & & 10 & 20 & 40 & 50 & 80 & 100 & 120 & 30 & 60 \\
\hline 2,4-Dichlorophenol & & 10 & 20 & 40 & 50 & 80 & 100 & 120 & 30 & 60 \\
\hline Benzoic Acid & & & 20 & 40 & 50 & 80 & 100 & 120 & 30 & 60 \\
\hline 1,2,4-Trichlorobenzene & & 10 & 20 & 40 & 50 & 80 & 100 & 120 & 30 & 60 \\
\hline Naphthalene & 1 & 10 & 20 & 40 & 50 & 80 & 100 & 120 & 30 & 60 \\
\hline alpha-Terpineol & & 10 & 20 & 40 & 50 & 80 & 100 & 120 & 30 & 60 \\
\hline 4-Chloroaniline & & 10 & 20 & 40 & 50 & 80 & 100 & 120 & 30 & 60 \\
\hline
\end{tabular}

Page 73 of 94 


\begin{tabular}{|c|c|c|c|c|c|c|c|c|c|c|}
\hline SW846 8270/EPA 625 & & & & & & & & & & \\
\hline Calibration Standard Concentration Levels ${ }^{\star}$ & & & & & & & & & & \\
\hline MEGA MIX & Level 1 & Level 2 & Level3 & Level 4\# & Level5 & Level 6 & Level 7 & Level 8 & Level 9 & Level 10 \\
\hline Hexachlorobutadiene & & 10 & 20 & 40 & 50 & 80 & 100 & 120 & 30 & 60 \\
\hline 4-Chloro-3-methylphenol & & 10 & 20 & 40 & 50 & 80 & 100 & 120 & 30 & 60 \\
\hline 2-Methylnaphthalene & 1 & 10 & 20 & 40 & 50 & 80 & 100 & 120 & 30 & 60 \\
\hline 1-Methylnaphthalene & 1 & 10 & 20 & 40 & 50 & 80 & 100 & 120 & 30 & 60 \\
\hline Hexachlorocyclopentadiene & -8 & 10 & 20 & 40 & 50 & 80 & 100 & 120 & 30 & 60 \\
\hline 2,3-Dichloroaniline & & 10 & 20 & 40 & 50 & 80 & 100 & 120 & 30 & 60 \\
\hline $2,4,6$-Trichlorophenol & & 10 & 20 & 40 & 50 & 80 & 100 & 120 & 30 & 60 \\
\hline 2,4,5-Trichlorophenol & 霖 & 10 & 20 & 40 & 50 & 80 & 100 & 120 & 30 & 60 \\
\hline 2-Chloronaphthalene & 1 & 10 & 20 & 40 & 50 & 80 & 100 & 120 & 30 & 60 \\
\hline o-Nitroaniline & & 10 & 20 & 40 & 50 & 80 & 100 & 120 & 30 & 60 \\
\hline m-Nitroaniline & +8 & 10 & 20 & 40 & 50 & 80 & 100 & 120 & 30 & 60 \\
\hline Dimethylphthalate & $1^{\star \star}$ & 10 & 20 & 40 & 50 & 80 & 100 & 120 & 30 & 60 \\
\hline 2,6-Dinitrotoluene & & 10 & 20 & 40 & 50 & 80 & 100 & 120 & 30 & 60 \\
\hline Acenaphthylene & 1 & 10 & 20 & 40 & 50 & 80 & 100 & 120 & 30 & 60 \\
\hline Acenaphthene & 1 & 10 & 20 & 40 & 50 & 80 & 100 & 120 & 30 & 60 \\
\hline 2,4-Dinitrophenol & & & 20 & 40 & 50 & 80 & 100 & 120 & 30 & 60 \\
\hline Dibenzofuran & & 10 & 20 & 40 & 50 & 80 & 100 & 120 & 30 & 60 \\
\hline 2,4-Dinitrotoluene & & 10 & 20 & 40 & 50 & 80 & 100 & 120 & 30 & 60 \\
\hline Diethylphthalate & $1^{\star \star \star}$ & 10 & 20 & 40 & 50 & 80 & 100 & 120 & 30 & 60 \\
\hline 4-Nitrophenol & & 10 & 20 & 40 & 50 & 80 & 100 & 120 & 30 & 60 \\
\hline Fluorene & 1 & 10 & 20 & 40 & 50 & 80 & 100 & 120 & 30 & 60 \\
\hline 4-Chlorophenyl phenyl ether & & 10 & 20 & 40 & 50 & 80 & 100 & 120 & 30 & 60 \\
\hline 2-Methyl-4,6-dinitrophenol & & 10 & 20 & 40 & 50 & 80 & 100 & 120 & 30 & 60 \\
\hline p-Nitroaniline & & 10 & 20 & 40 & 50 & 80 & 100 & 120 & 30 & 60 \\
\hline Diphenylamine & & 10 & 20 & 40 & 50 & 80 & 100 & 120 & 30 & 60 \\
\hline 1,2-Diphenylhydrazine & & 10 & 20 & 40 & 50 & 80 & 100 & 120 & 30 & 60 \\
\hline 4-Bromophenyl phenyether & & 10 & 20 & 40 & 50 & 80 & 100 & 120 & 30 & 60 \\
\hline Hexachlorobenzene & & 10 & 20 & 40 & 50 & 80 & 100 & 120 & 30 & 60 \\
\hline Pentachlorophenol & & 10 & 20 & 40 & 50 & 80 & 100 & 120 & 30 & 60 \\
\hline n-Octadecane & & 10 & 20 & 40 & 50 & 80 & 100 & 120 & 30 & 60 \\
\hline Phenanthrene & 1 & 10 & 20 & 40 & 50 & 80 & 100 & 120 & 30 & 60 \\
\hline Anthracene & 1 & 10 & 20 & 40 & 50 & 80 & 100 & 120 & 30 & 60 \\
\hline Di-n-butylphthalate & $1^{\star \star}$ & 10 & 20 & 40 & 50 & 80 & 100 & 120 & 30 & 60 \\
\hline Fluoranthene & 1 & 10 & 20 & 40 & 50 & 80 & 100 & 120 & 30 & 60 \\
\hline Pyrene & 1 & 10 & 20 & 40 & 50 & 80 & 100 & 120 & 30 & 60 \\
\hline Butylbenzylphthalate & $1^{\star \star}$ & 10 & 20 & 40 & 50 & 80 & 100 & 120 & 30 & 60 \\
\hline Benzo(a)anthracene & 1 & 10 & 20 & 40 & 50 & 80 & 100 & 120 & 30 & 60 \\
\hline Chrysene & 1 & 10 & 20 & 40 & 50 & 80 & 100 & 120 & 30 & 60 \\
\hline bis (2-Ethylhexyl) phthalate & 1 & 10 & 20 & 40 & 50 & 80 & 100 & 120 & 30 & 60 \\
\hline Di-n-octylphthalate & $1^{\star \star}$ & 10 & 20 & 40 & 50 & 80 & 100 & 120 & 30 & 60 \\
\hline
\end{tabular}

Page 74 of 94 


\begin{tabular}{|c|c|c|c|c|c|c|c|c|c|c|}
\hline \multirow{2}{*}{\multicolumn{11}{|c|}{$\begin{array}{l}\text { SW846 8270/EPA 625 } \\
\text { Calibration Standard Concentration Levels }\end{array}$}} \\
\hline & & & & & & & & & & \\
\hline MEGA MIX & Level 1 & Level 2 & Level3 & Level 4\# & Level 5 & Level 6 & Level 7 & Level 8 & Level 9 & Level 10 \\
\hline Benzo(b)fluoranthene & 1 & 10 & 20 & 40 & 50 & 80 & 100 & 120 & 30 & 60 \\
\hline Benzo(k)fluoranthene & 1 & 10 & 20 & 40 & 50 & 80 & 100 & 120 & 30 & 60 \\
\hline Benzo(a)pyrene & 1 & 10 & 20 & 40 & 50 & 80 & 100 & 120 & 30 & 60 \\
\hline Indeno-(1,2,3-cd)pyrene & 1 & 10 & 20 & 40 & 50 & 80 & 100 & 120 & 30 & 60 \\
\hline Dibenzo(a,h)anthracene & 1 & 10 & 20 & 40 & 50 & 80 & 100 & 120 & 30 & 60 \\
\hline Benzo(ghi)perylene & 1 & 10 & 20 & 40 & 50 & 80 & 100 & 120 & 30 & 60 \\
\hline m-Dinitrobenzene & & 10 & 20 & 40 & 50 & 80 & 100 & 120 & 30 & 60 \\
\hline $2,3,4,6$-Tetrachlorophenol & & $\overline{10}$ & 20 & 40 & 50 & 80 & 100 & 120 & 30 & 60 \\
\hline Dinoseb & & 10 & 20 & 40 & 50 & 80 & 100 & 120 & 30 & 60 \\
\hline Carbazole & 1 & 10 & 20 & 40 & 50 & 80 & 100 & 120 & 30 & 60 \\
\hline p-Benzoquinone & & 10 & 20 & 40 & 50 & 80 & 100 & 120 & 30 & 60 \\
\hline Methoxychlor & & 10 & 20 & 40 & 50 & 80 & 100 & 120 & 30 & 60 \\
\hline p-Toluidine & & 10 & 20 & 40 & 50 & 80 & 100 & 120 & 30 & 60 \\
\hline m-Toluidine & & 10 & 20 & 40 & 50 & 80 & 10 & 120 & 30 & 60 \\
\hline 1,4-Dinitrobenzene & & 10 & 20 & 40 & 50 & 80 & 100 & 120 & 30 & 60 \\
\hline 2-Ethoxyethanol & & 10 & 20 & 40 & 50 & 80 & 100 & 120 & 30 & 60 \\
\hline Phthalic anhydride & & 10 & 20 & 40 & 50 & 80 & 100 & 120 & 30 & 60 \\
\hline Methylenebis(2-chloroaniline) & & 10 & 20 & 40 & 50 & 80 & 100 & 120 & 30 & 60 \\
\hline Dibenzo(a,e)pyrene & & 10 & 20 & 40 & 50 & 80 & 100 & 120 & 30 & 60 \\
\hline
\end{tabular}

\begin{tabular}{|c|c|c|c|c|c|c|c|c|c|c|}
\hline \multicolumn{11}{|l|}{ SW846 8270/EPA 625} \\
\hline \multicolumn{11}{|l|}{ Calibration Standard Concentration Levels ${ }^{\star}$} \\
\hline AP MIX & $\overline{\text { Level } 1}$ & Level 2 & Level3 & Level 4\# & Level 5 & Level 6 & Level 7 & Level 8 & Level 9 & Level 10 \\
\hline Benzaldehyde & & 10 & 20 & 40 & 50 & 80 & 100 & 120 & 30 & 60 \\
\hline Acetophenone & & 10 & 20 & 40 & 50 & 80 & 100 & 120 & 30 & 60 \\
\hline Caprolactam & & 10 & 20 & 40 & 50 & 80 & 100 & 120 & 30 & 60 \\
\hline 1,1'-Biphenyl & & 10 & 20 & 40 & 50 & 80 & 100 & 120 & 30 & 60 \\
\hline Atrazine & & 10 & 20 & 40 & 50 & 80 & 100 & 120 & 30 & 60 \\
\hline Benzidine & & 10 & 20 & 40 & 50 & 80 & 100 & 120 & 30 & 60 \\
\hline 3,3'-Dichlorobenzidene & & 10 & 20 & 40 & 50 & 80 & 100 & 120 & 30 & 60 \\
\hline 1,4-Dioxane & & 10 & 20 & 40 & 50 & 80 & 100 & 120 & 30 & 60 \\
\hline Methyl methacrylate & & 10 & 20 & 40 & 50 & 80 & 100 & 120 & 30 & 60 \\
\hline Ethyl methacrylate & & 10 & 20 & 40 & 50 & 80 & 100 & 120 & 30 & 60 \\
\hline 2-Picoline & & 10 & 20 & 40 & 50 & 80 & 100 & 120 & 30 & 60 \\
\hline N-Nitrosomethylethylamine & & 10 & 20 & 40 & 50 & 80 & 100 & 120 & 30 & 60 \\
\hline 2-Butoxyethanol & & 10 & 20 & 40 & 50 & 80 & 100 & 120 & 30 & 60 \\
\hline Methyl methanesulfonate & & 10 & 20 & 40 & 50 & 80 & 100 & 120 & 30 & 60 \\
\hline N-Nitrosodiethylamine & & 10 & 20 & 40 & 50 & 80 & 100 & 120 & 30 & 60 \\
\hline Ethyl methanesulfonate & & 10 & 20 & 40 & 50 & 80 & 100 & 120 & 30 & 60 \\
\hline Pentachloroethane & & 10 & 20 & 40 & 50 & 80 & 100 & 120 & 30 & 60 \\
\hline N-Nitrosopyrrolidine & & 10 & 20 & 40 & 50 & 80 & 100 & 120 & 30 & 60 \\
\hline N-Nitrosomorpholine & & 10 & 20 & 40 & 50 & 80 & 100 & 120 & 30 & 60 \\
\hline o-Toluidine & & 10 & 20 & 40 & 50 & 80 & 100 & 120 & 30 & 60 \\
\hline N-Nitrosopiperidine & & 10 & 20 & 40 & 50 & 80 & 100 & 120 & 30 & 60 \\
\hline a,a-Dimethylphenethylamine & & 10 & 20 & 40 & 50 & 80 & 100 & 120 & 30 & 60 \\
\hline 2,6-Dichlorophenol & & 10 & 20 & 40 & 50 & 80 & 100 & 120 & 30 & 60 \\
\hline
\end{tabular}

Page 75 of 94 


\begin{tabular}{|c|c|c|c|c|c|c|c|c|c|c|}
\hline \multirow{2}{*}{\begin{tabular}{|l} 
SW846 8270/EPA 625 \\
Calibration Standard Concentration Levels*
\end{tabular}} & \multirow[b]{3}{*}{ Level 1} & \multirow[b]{3}{*}{$\overline{\text { Level } 2}$} & \multirow[b]{3}{*}{ Level 3} & \multirow[b]{3}{*}{ Level 4A } & \multirow[b]{3}{*}{ Level 5} & \multirow[b]{3}{*}{ Level 6 } & \multirow[b]{3}{*}{ Level 7} & \multirow[b]{3}{*}{ Level 8 } & \multirow[b]{3}{*}{ Level 9} & \multirow[b]{3}{*}{ Level 10} \\
\hline & & & & & & & & & & \\
\hline AP MIX & & & & & & & & & & \\
\hline Hexachloropropene & & 10 & 20 & 40 & 50 & $\overline{80}$ & 100 & 120 & 30 & 60 \\
\hline N-Nitrosodi-n-butylamine & & 10 & 20 & 40 & 50 & 80 & 100 & 120 & 30 & 60 \\
\hline Safrole & & 10 & 20 & 40 & 50 & 80 & 100 & 120 & 30 & 60 \\
\hline 1,2,4,5-Tetrachlorobenzene & & 10 & 20 & 40 & 50 & 80 & 100 & 120 & 30 & 60 \\
\hline Isosafrole & & 10 & 20 & 40 & 50 & 80 & 100 & 120 & 30 & 60 \\
\hline 1,4-Naphthoquinone & & 10 & 20 & 40 & 50 & 80 & 100 & 120 & 30 & 60 \\
\hline Pentachlorobenzene & & 10 & 20 & 40 & 50 & 80 & 100 & 120 & 30 & 60 \\
\hline 1-Naphthylamine & & 10 & 20 & 40 & 50 & 80 & 100 & 120 & 30 & 60 \\
\hline 2-Naphthylamine & & 10 & 20 & 40 & 50 & 80 & 100 & 120 & 30 & 60 \\
\hline 5-Nitro-o-toluidine & & 10 & 20 & 40 & 50 & 80 & 100 & 120 & 30 & 60 \\
\hline 1,3,5-Trinitrobenzene & & 10 & 20 & 40 & 50 & 80 & 100 & 120 & 30 & 60 \\
\hline Phenacetin & & 10 & 20 & 40 & 50 & 80 & 100 & 120 & 30 & 60 \\
\hline Diallate & & 10 & 20 & 40 & 50 & 80 & 100 & 120 & 30 & 60 \\
\hline cis-Diallate & & 1.5 & 3 & 6 & 7.5 & 12 & 15 & 18 & 4.5 & 9 \\
\hline trans-Diallate & & 8.5 & 17 & 34 & 42 & 68 & 85 & 102 & 25.5 & 51 \\
\hline 4-Aminobiphenyl & & 10 & 20 & 40 & 50 & 80 & 100 & 120 & 30 & 60 \\
\hline Pentachloronitrobenzene & & 10 & 20 & 40 & 50 & 80 & 100 & 120 & 30 & 60 \\
\hline Pronamide & & 10 & 20 & 40 & 50 & 80 & 100 & 120 & 30 & 60 \\
\hline 4-Nitroquinoline-1-oxide & & 10 & 20 & 40 & 50 & 80 & 100 & 120 & 30 & 60 \\
\hline Methapyrilene & & 10 & 20 & 40 & 50 & 80 & 100 & 120 & 30 & 60 \\
\hline Isodrin & & 10 & 20 & 40 & 50 & 80 & 100 & 120 & 30 & 60 \\
\hline Aramite & & 10 & 20 & 40 & 50 & 80 & 100 & 120 & 30 & 60 \\
\hline Kepone & & 10 & 20 & 40 & 50 & 80 & 100 & 120 & 30 & 60 \\
\hline p-(Dimethylamino)azobenzene & & 10 & 20 & 40 & 50 & 80 & 100 & 120 & 30 & 60 \\
\hline Chlorobenzilate & & 10 & 20 & 40 & 50 & 80 & 100 & 120 & 30 & 60 \\
\hline 3,3'-Dimethylbenzidine & & 10 & 20 & 40 & 50 & 80 & 100 & 120 & 30 & 60 \\
\hline 2-Acetylaminofluorene & & 10 & 20 & 40 & 50 & 80 & 100 & 120 & 30 & 60 \\
\hline 7,12-Dimethylbenz(a)anthracene & & 10 & 20 & 40 & 50 & 80 & 100 & 120 & 30 & 60 \\
\hline 3-Methylcholanthrene & & 10 & 20 & 40 & 50 & 80 & 100 & 120 & 30 & 60 \\
\hline
\end{tabular}

\begin{tabular}{|c|c|c|c|c|c|c|c|c|c|c|}
\hline \multirow{2}{*}{\multicolumn{11}{|c|}{\begin{tabular}{|l|} 
SW846 8270/EPA 625 \\
Calibration Standard Concentration Levels*
\end{tabular}}} \\
\hline & & & & & & & & & & \\
\hline & Level 1 & Level 2 & Level 3 & Level 4\# & Level 5 & Level 6 & Level 7 & Level 8 & Level 9 & Level 10 \\
\hline Hexachlorophene & & 500 & 1000 & 1250 & 1500 & 1750 & 2000 & & & \\
\hline p-Phenylenediamine & & 500 & 1000 & 1250 & 1500 & 1750 & 2000 & & & \\
\hline
\end{tabular}

Page 76 of 94 


\begin{tabular}{|c|c|c|c|c|c|c|c|c|c|c|}
\hline \multirow{2}{*}{\begin{tabular}{|l} 
SW846 8270/EPA 625 \\
Calibration Standard Concentration Levels* \\
\end{tabular}} & \multirow[b]{3}{*}{ Level 1} & \multirow[b]{3}{*}{ Level 2} & \multirow[b]{3}{*}{ Level3 } & \multirow[b]{3}{*}{ Level 4ff } & \multirow[b]{3}{*}{ Level 5} & \multirow[b]{3}{*}{ Level 6} & \multirow[b]{3}{*}{ Level 7} & \multirow[b]{3}{*}{ Level 8} & \multirow[b]{3}{*}{ Level 9} & \multirow[b]{3}{*}{ Level 10} \\
\hline & & & & & & & & & & \\
\hline PEST MIX & & & & & & & & & & \\
\hline bis(Chloromethyl)ether & & 10 & 20 & 40 & 50 & 80 & 100 & 120 & 30 & 60 \\
\hline Tributylphosphate & & 10 & 20 & 40 & 50 & 80 & 100 & 120 & 30 & 60 \\
\hline Triethylphosphorothioate & & 10 & 20 & 40 & 50 & 80 & 100 & 120 & 30 & 60 \\
\hline Thionazin & & 10 & 20 & 40 & 50 & 80 & 100 & 120 & 30 & 60 \\
\hline Sulfotepp & & 10 & 20 & 40 & 50 & 80 & 100 & 120 & 30 & 60 \\
\hline Phorate & & 10 & 20 & 40 & 50 & 80 & 100 & 120 & 30 & 60 \\
\hline Dimethoate & & 10 & 20 & 40 & 50 & 80 & 100 & 120 & 30 & 60 \\
\hline Disulfoton & & 10 & 20 & 40 & 50 & 80 & 100 & 120 & 30 & 60 \\
\hline Methyl parathion & & 10 & 20 & 40 & 50 & 80 & 100 & 120 & 30 & 60 \\
\hline Famphur & & 10 & 20 & 40 & 50 & 80 & 100 & 120 & 30 & 60 \\
\hline Parathion & & 10 & 20 & 40 & 50 & 80 & 100 & 120 & 30 & 60 \\
\hline
\end{tabular}

\begin{tabular}{|c|c|c|c|c|c|c|c|c|c|c|}
\hline \multicolumn{11}{|l|}{ SW846 8270/EPA 625} \\
\hline \multicolumn{11}{|l|}{ Calibration Standard Concentration Levels ${ }^{\star}$} \\
\hline NEVADA MIX & Level 1 & Level 2 & Level 3 & Level 4\# & Level 5 & Level 6 & Level 7 & Level 8 & Level 9 & Level 10 \\
\hline bis(Chloromethyl)ether & & 10 & 20 & 40 & 50 & 80 & 100 & 120 & 30 & 60 \\
\hline 4-Chlorothiophenol & & 10 & 20 & 40 & 50 & 80 & 100 & 120 & 30 & 60 \\
\hline 4-Chlorothioanisole & & 10 & 20 & 40 & 50 & 80 & 100 & 120 & 30 & 60 \\
\hline Phthalic acid & & 10 & 20 & 40 & 50 & 80 & 100 & 120 & 30 & 60 \\
\hline Hydroxymethyl phthalimide & & 10 & 20 & 40 & 50 & 80 & 100 & 120 & 30 & 60 \\
\hline Diphenyl sulfide & & 10 & 20 & 40 & 50 & 80 & 100 & 120 & 30 & 60 \\
\hline Diphenyl disulfide & & 10 & 20 & 40 & 50 & 80 & 100 & 120 & 30 & 60 \\
\hline Phenyl sulfone & & 10 & 20 & 40 & 50 & 80 & 100 & 120 & 30 & 60 \\
\hline Octachlorostyrene & & 10 & 20 & 40 & 50 & 80 & 100 & 120 & 30 & 60 \\
\hline Thiophenol & 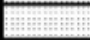 & 10 & 20 & 40 & 50 & 80 & 100 & 120 & 30 & 60 \\
\hline 2,2'-Dichlorobenzil & & 10 & 20 & 40 & 50 & 80 & 100 & 120 & 30 & 60 \\
\hline bis(p-Chlorophenyl)disulfide & & 10 & 20 & 40 & 50 & 80 & 100 & 120 & 30 & 60 \\
\hline bis(p-Chlorophenyl)sulfone & & 10 & 20 & 40 & 50 & 80 & 100 & 120 & 30 & 60 \\
\hline
\end{tabular}

All values are $\mathrm{mg} / \mathrm{L}$ without the prep factor.

\# Indicates the calibration verification concentration level used

* Usual calibration levels using SCAN methodology

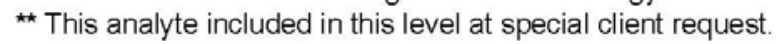

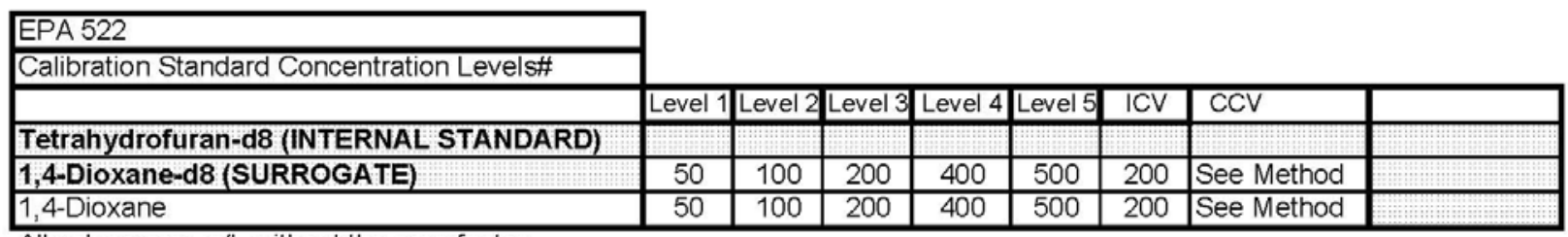

All values are $\mathrm{ug} / \mathrm{L}$ without the prep factor.

\# Usual calibration levels using SIM methodology

Page 77 of 94 


\begin{tabular}{|c|c|c|c|c|c|c|c|c|c|c|}
\hline \multirow{2}{*}{\multicolumn{11}{|c|}{$\begin{array}{l}\text { SW846 8270SIM } \\
\text { Calibration Standard Concentration Levels }\end{array}$}} \\
\hline & & & & & & & & & & \\
\hline MEGASIM analytes (A) & Level 1 & Level 2 & Level 3 & Level 4 & Level 5 & evel 6f & Level 7 & Level 8 & Level 9 & Level 10 \\
\hline \multicolumn{11}{|c|}{ 1,4-Dichlorobenzene-d4 (INTERNAL STANDARD) } \\
\hline \multicolumn{11}{|c|}{ Naphthalene-d8 (INTERNAL STANDARD) } \\
\hline \multicolumn{11}{|c|}{ Acenaphthene-d10 (INTERNAL STANDARD) } \\
\hline \multicolumn{11}{|c|}{ Phenanthrene-d10 (INTERNAL STANDARD) } \\
\hline \multicolumn{11}{|l|}{ Chrysene-d12 (INTERNAL STANDARD) } \\
\hline \multicolumn{11}{|c|}{ Perylene-d12 (INTERNAL STANDARD) } \\
\hline 5-alpha-Androstane (SURROGATE) & $\$ 0.1$ & 0.2 & 0.5 & 1 & 2 & 5 & 10 & 20 & & \\
\hline \$N-Methyl-N-nitrosomethylamine & & 0.2 & 0.5 & 1 & 2 & 5 & 10 & 20 & & \\
\hline Sbis(2-Chloroethyl)ether & 0.1 & 0.2 & 0.5 & 1 & 2 & 5 & 10 & 20 & & \\
\hline \$N-Nitrosodipropylamine & 0.1 & 0.2 & 0.5 & 1 & 2 & 5 & 10 & 20 & & \\
\hline Naphthalene & $\$ 0.1$ & 0.2 & 0.5 & 1 & 2 & 5 & 10 & 20 & & \\
\hline 2-Methylnaphthalene & $\$ 0.1$ & 0.2 & 0.5 & 1 & 2 & 5 & 10 & 20 & & \\
\hline 1-Methylnaphthalene & $\$ 0.1$ & 0.2 & 0.5 & 1 & 2 & 5 & 10 & 20 & & \\
\hline 2-Chloronaphthalene & $\$ 0.1$ & 0.2 & 0.5 & 1 & 2 & 5 & 10 & 20 & & \\
\hline Acenaphthylene & $\$ 0.1$ & 0.2 & 0.5 & 1 & 2 & 5 & 10 & 20 & & \\
\hline Acenaphthene & $\$ 0.1$ & 0.2 & 0.5 & 1 & 2 & 5 & 10 & 20 & & \\
\hline Fluorene & $\$ 0.1$ & 0.2 & 0.5 & 1 & 2 & 5 & 10 & 20 & & \\
\hline Phenanthrene & $\$ 0.1$ & 0.2 & 0.5 & 1 & 2 & 5 & 10 & 20 & & \\
\hline Anthracene & $\$ 0.1$ & 0.2 & 0.5 & 1 & 2 & 5 & 10 & 20 & & \\
\hline Fluoranthene & $\$ 0.1$ & 0.2 & 0.5 & 1 & 2 & 5 & 10 & 20 & & \\
\hline Pyrene & $\$ 0.1$ & 0.2 & 0.5 & 1 & 2 & 5 & 10 & 20 & & \\
\hline Benzo(a)anthracene & $\$ 0.1$ & 0.2 & 0.5 & 1 & 2 & 5 & 10 & 20 & & \\
\hline Chrysene & $\$ 0.1$ & 0.2 & 0.5 & 1 & 2 & 5 & 10 & 20 & & \\
\hline Benzo(b)fluoranthene & $\$ 0.1$ & 0.2 & 0.5 & 1 & 2 & 5 & 10 & 20 & & \\
\hline Benzo(k)fluoranthene & $\$ 0.1$ & 0.2 & 0.5 & 1 & 2 & 5 & 10 & 20 & & \\
\hline Benzo(a)pyrene & $\$ 0.1$ & 0.2 & 0.5 & 1 & 2 & 5 & 10 & 20 & & \\
\hline Indeno-(1,2,3-cd)pyrene & $\$ 0.1$ & 0.2 & 0.5 & 1 & 2 & 5 & 10 & 20 & & \\
\hline Dibenzo $(\mathrm{a}, \mathrm{h})$ anthracene & $\$ 0.1$ & 0.2 & 0.5 & 1 & 2 & 5 & 10 & 20 & & \\
\hline Benzo(ghi)perylene & $\$ 0.1$ & 0.2 & 0.5 & 1 & 2 & 5 & 10 & 20 & & \\
\hline
\end{tabular}

$\$$ By special request - Not for regulatory purposes

SW846 8270SIM

Calibration Standard Concentration Levels ${ }^{*}$

APSIM analytes $(A)$

\$N-Nitrosodimethylamine

\$N-Nitrosopyrrolidine

\$N-Nitrosodi-n-butylamine

\$Benzidine

$\$ 3,3^{\prime}$-Dichlorobenzidine

$\$$ By special request - Not for regulatory purposes

All values are $\mathrm{mg} / \mathrm{L}$ without prep factor.

\# indicates the calibraton verification concentration level used.

* Usual calibration levels using SIM methodology

(10/16/Full list)

Page 78 of 94 
Calibration History Report MSD4

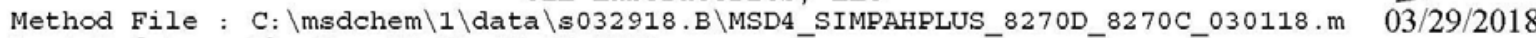

Last Update : Thu Mar 01 12:09:52 2018

Integrator : (RTE Integrator)

Response via : Initial Calibration

Cal Lvl:1 Amt:0.10 Last Updated with: C: \msdchem \1 data \s030118.B \s4c0102.D

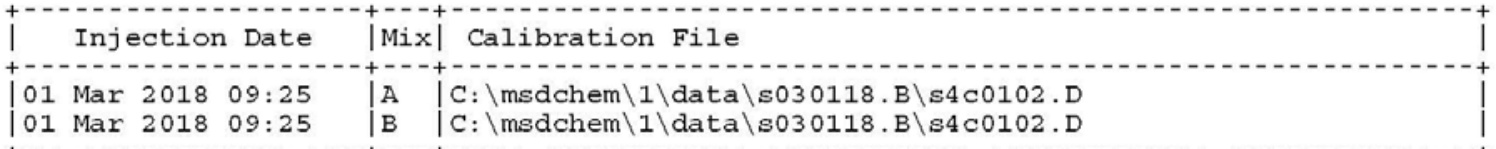

Cal Lvl:2 Amt:0.20 None of the compounds use this level.

| Injection Date |Mix| Calibration File

Cal Lvl:3 Amt:0.50 Last Updated with: C: $\backslash$ msdchem $\backslash 1 \backslash$ data $\backslash$ s030118.B $\backslash$ S4C0103.D

\begin{tabular}{l}
$\mid$ Injection Date $\mid$ Mix| Calibration File \\
\hline 01 Mar $201809: 53 \quad \mid$ A $\mid$ C: $\backslash$ msdchem $\backslash 1 \backslash$ data $\backslash 5030118 . B \backslash S 4 c 0103 . D$ \\
01
\end{tabular}

Cal Lvl:4 Amt:1.00 Last Updated with: C: \msdchem \1\data \s030118.B \s4c0104.D

| Injection Date $\mid$ Mix| Calibration File

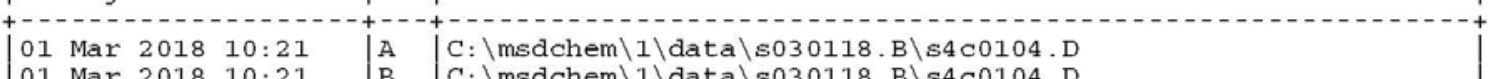

01 Mar $201810: 21 \quad|B| C: \backslash m s d c h e m \backslash 1 \backslash$ data $\backslash$ s030118.B $\backslash s 4 c 0104 . D$

+

Cal Lvl:5 Amt:2.00 None of the compounds use this level.

| Injection Date |Mix| Calibration File

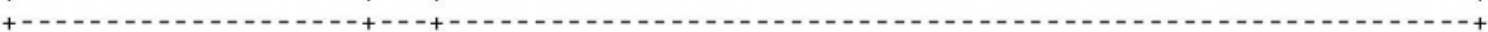

Cal Lvl:6 Amt:5.00 Last Updated with: C: \msdchem \1\data \s030118.B \s4c0105.D

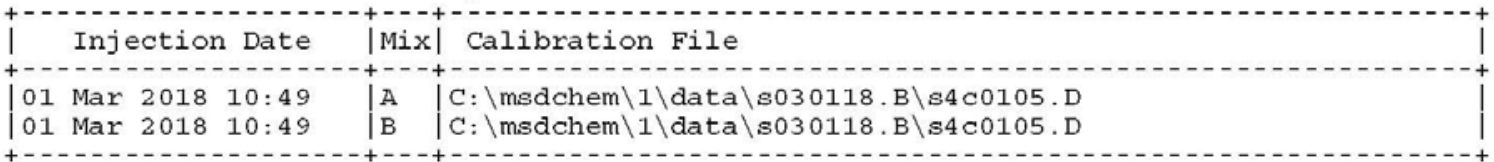

Cal Lv1:7 Amt:10.00 Last Updated with: C:\msdchem \1\data \s030118.B \s4c0106.D

| Injection Date |Mix| Calibration File

01 Mar 2018 11:17 |A |C: \msdchem $\backslash 1 \backslash$ data $\backslash$ s030118.B $\backslash$ s4c0106.D

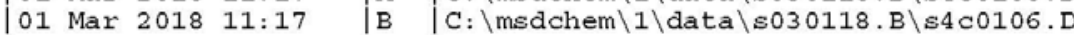

t- Mar 2018 11:17

Cal Lvl:8 Amt:20.00 Last Updated with: C: \msdchem $\backslash 1 \backslash$ data $\backslash$ s030118.B $\backslash$ s4 C0107.D

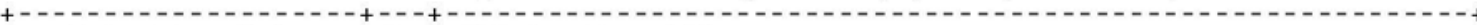
| Injection Date $\mid$ Mix| Calibration

01 Mar 2018 11:46 |A |C: \msdchem $\backslash 1 \backslash$ data $\backslash 5030118 . \mathrm{B} \backslash$ s4c0107.

01 Mar 2018 11:46 |B $\mid \mathrm{C}: \backslash$ msdchem $\backslash 1 \backslash$ data $\backslash 5030118 . \mathrm{B} \backslash \mathrm{s} 4 \mathrm{c0107.D}$

+

MSD4_SIMPAH...70C_030118.m Thu Mar 29 15:33:19 2018

MSD4_SIMPAH...70C_030118.m Thu Mar 29 15:33:16 2018

Page 79 of 94 


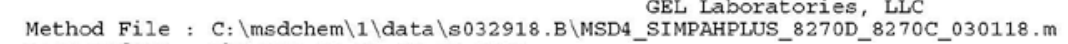

Last Update : Thu Mar 01 12:09:52 2018

Integrator : (RTE Integrator)

Response via : Initial Calibration

For Linear Calibration: $\mathrm{x}=$ concentration ratio, $\mathrm{y}=$ response ratio, $\mathrm{y}=\mathrm{b}+\mathrm{m} 1(\mathrm{x})+\mathrm{m} 2(\mathrm{xE} 2)$

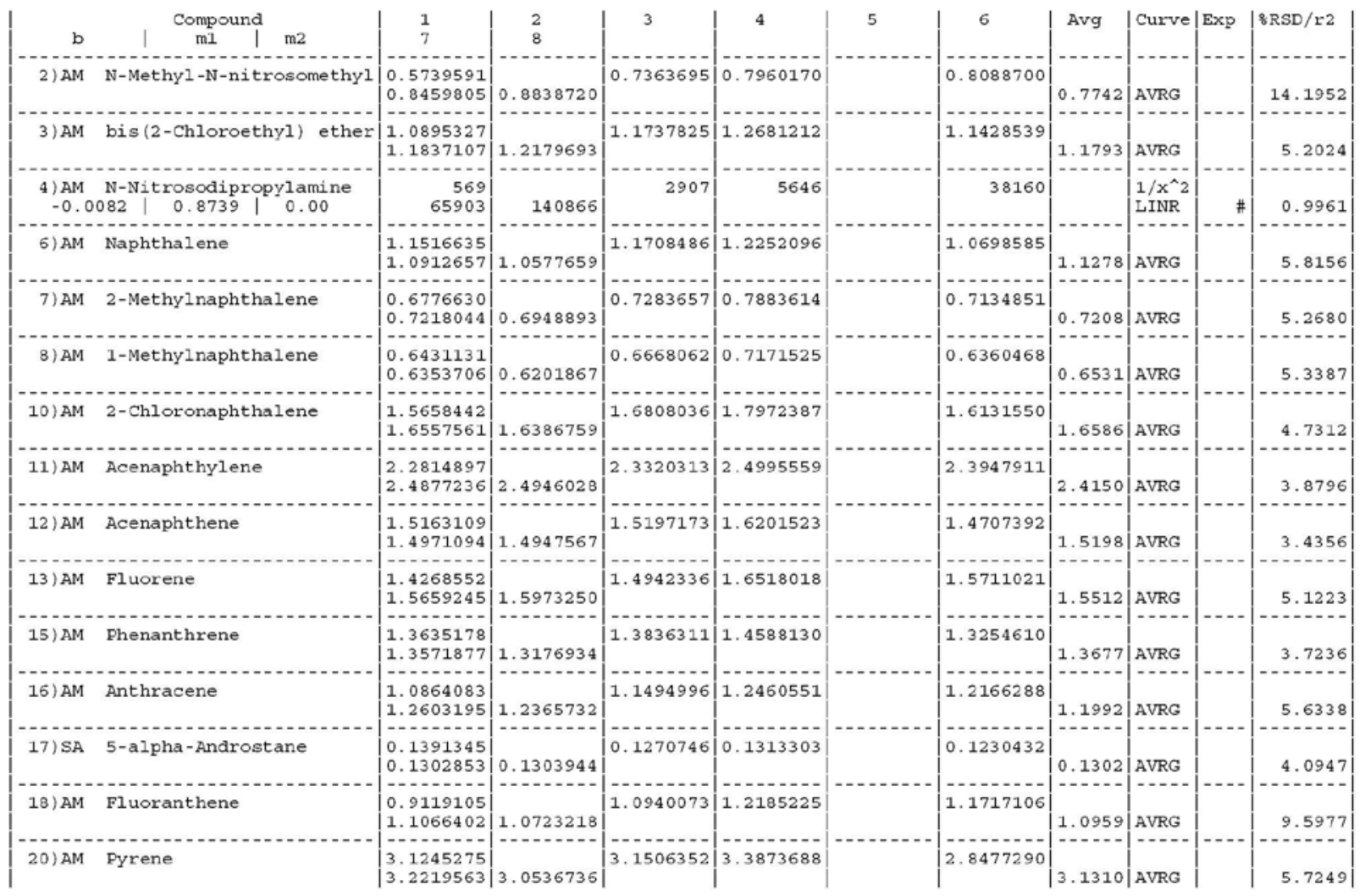

MSD4_SIMPAH...70C_030118.m Thu Mar 29 15:33:16 2018

Page: 1 
Response Factor Report MSD4

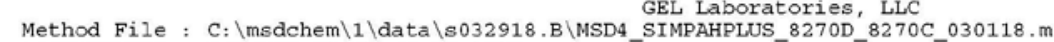

Last Update; Thu Mar 01 12:09:52 2018

Integrator : (RTE Integrator)

Response via : Initial Calibration

For Linear Calibration: $\mathrm{x}=$ concentration ratio, $\mathrm{y}=$ response ratio, $\mathrm{y}=\mathrm{b}+\mathrm{m} 1(\mathrm{x})+\mathrm{m} 2(\mathrm{xE} 2)$

\begin{tabular}{|c|c|c|c|c|c|c|c|c|c|c|}
\hline$\underset{\mathrm{m} 1}{\text { Compound }} \mathrm{m} 2$ & $\begin{array}{l}1 \\
7\end{array}$ & $\begin{array}{l}2 \\
8\end{array}$ & 3 & 4 & 5 & 6 & Avg & | Curve | & Exp & $8 \mathrm{RSD} / \mathrm{r} 2$ \\
\hline 21) AM Benzo(a) anthracene & $\mid \begin{array}{l}1.5844378 \\
1.5220261\end{array}$ & 1.5607207 & 1.3284936 & $|-4619565|$ & & $|1.4448617|$ & 1.4837 & AVRG & & 6.2950 \\
\hline 22) AM Chrysene & $\mid \begin{array}{l}1.5622638 \\
1.5055427\end{array}$ & 1.4872197 & 1.5201452 & 1.6746627 & & 1.4589952 & 1.5348 & AVRG & & 4. 9959 \\
\hline 24) AM Benzo(b) fluoranthene & $\left|\begin{array}{l}\mid-1 .-1 \\
1.6594033 \\
1.9078847\end{array}\right|$ & 2.0213493 & 1.7846222 & 2.0141781 & & 1.8278896 & 1.8692 & |AVRG & & 7.5103 \\
\hline 25)AM Benzo(k) fluoranthene & 1.5990613 & & 1.7159828 & 1.9375277 & & $|1.8381171|$ & & & & \\
\hline & 1.9338279 & 2.0074461 & & & & & 1.8387 & AVRG & & 8.4179 \\
\hline 26) AM Benzo(a) pyrene & $\mid \begin{array}{l}1.3040563 \\
1.5228487\end{array}$ & 1.6607073 & 1.2548675 & 1.4124945 & & 1.3936668 & 1.4248 & AVRG & & 10.4029 \\
\hline 27) AM Indeno(1,2,3-cd) pyrene & $\left|\begin{array}{|c|}\mid \\
0.9520617 \\
0.9880882\end{array}\right|$ & 1.1068396 & 0.8201518 & 0.8560035 & & $|0.8268013|$ & 0.9250 & $-\cdots$ & & 12.1520 \\
\hline 28) AM Dibenzo $(\mathrm{a}, \mathrm{h})$ anthracene & 0.9989943 & & 0.7383126 & 0.7585290 & & 0.7573592 & & & & \\
\hline- & 0.9052565 & 0.9592423 & $\ldots-1$ & & & & 0.8529 & AVRG & & 13.5267 \\
\hline 29) AM Benzo(ghi) perylene & $\mid \begin{array}{l}1.2738854 \\
1.1258160\end{array}$ & 1.1435850 & 1.0269497 & 1.0943731 & & 0.9550384 & 1.1033 & AVRG & & 9.8559 \\
\hline $\begin{array}{l}\text { 31) BM N-Nitrosodiethylamine } \\
-0.0055|0.5325| 0.00\end{array}$ & $\begin{array}{r}332 \\
41143\end{array}$ & 88698 & 1686 & 3274 & & 23295 & & $\begin{array}{l}-\cdot-- \\
1 / x^{\wedge} 2 \\
\text { LINR }\end{array}$ & $\#$ & 0.9916 \\
\hline 32) BM N-Nitrosopyrrolidine & $\left|\begin{array}{l}0.2612503 \\
0.5501720\end{array}\right|$ & 0.5935886 & 0.3831698 & 0.4514570 & & 0.5224042 & 0.4603 & AVRG & \# & 26.6668 \\
\hline - & (-......- & - & $-1-1-1$ & 17 & & 21106 & $\cdots$ & $-1 \hat{x}^{3}$ & & - \\
\hline $\begin{array}{l}\text { 34) BM N-Nitrosodi-n-butylamine } \\
-0.0021|0.2169| 0.00\end{array}$ & $\begin{array}{r}462 \\
54558\end{array}$ & 120445 & 2316 & 4473 & & 31196 & & $\begin{array}{l}1 / \mathrm{x}^{\wedge} \\
\mathrm{LINR}\end{array}$ & \# & 0.9940 \\
\hline $\begin{array}{l}\text { 36) BM Benzidine } \\
-0.1531 \quad 0.3566 \mid 0.00\end{array}$ & 230269 & 578183 & 6072 & 16305 & & 176378 & - & LINR & \# & 0.9976 \\
\hline $\begin{array}{l}\text { 38) BM } 3,3^{\prime} \text {-Dichlorobenzidine } \\
-0.0057 \text { | } 0.3927 \mid 0.00\end{array}$ & $\begin{array}{r}165 \\
19174\end{array}$ & & 689 & 1601 & & 14438 & & $1 / x$ & & 0.9916 \\
\hline
\end{tabular}

\#) = Out of Range (\$) = Individual RF out of Range

AVRG $=$ Average, $\operatorname{LINR}=$ Linear Regression, $1 / x=$ the inverse of concentration, $1 / x^{\wedge} 2=$ the inverse square of concentration 
Continuing Calibration Summary

Instrument ID: MSD4.I

Data File: s030118.B\s4c0108.D

Lab Sample ID WBN180301-89

Quant Type ISTD
Client SDG: $\quad 446517$

Injection Date: $\quad$ 01-MAR-18 12:16

Init. Cal. Date(s) : 01-MAR-18 09:25 - 01-MAR-18 11:4

Method: s030118.BLMSD4_SIMPAHPLUS_8270D_8270C

Method Update: 01-MAR-18 12:09

\begin{tabular}{|l|r|r|r|r|r|r|r|r|r|}
\hline Compound & $\begin{array}{c}\text { AVERF / } \\
\text { Amount }\end{array}$ & $\begin{array}{c}\text { RF } \\
\text { CCV }\end{array}$ & $\begin{array}{c}\text { Nominal } \\
\text { CCV }\end{array}$ & Min RF & $\begin{array}{c}\text { RF } \\
\text { Q }\end{array}$ & $\begin{array}{c}\text { \%D / } \\
\text { \%Drift }\end{array}$ & $\begin{array}{c}\text { Max } \\
\text { Drift } \\
\text { Q }\end{array}$ & $\begin{array}{c}\text { Curve } \\
\text { Type }\end{array}$ \\
\hline S 5-alpha-Androstane & 0.1302 & 0.11819 & & .01 & & -9.22427 & 30 & & Averaged \\
\hline Naphthalene & 1.1278 & 1.06558 & .7 & & -5.51694 & 30 & & Averaged \\
\hline Acenaphthene & 1.5198 & 1.42055 & .9 & & -6.53046 & 30 & & Averaged \\
\hline Fluorene & 1.5512 & 1.51179 & .9 & & -2.54061 & 30 & & Averaged \\
\hline Anthracene & 1.1992 & 1.18409 & .7 & & -1.26001 & 30 & & Averaged \\
\hline Fluoranthene & 1.0959 & 1.10453 & .6 & & 0.78748 & 30 & & Averaged \\
\hline Pyrene & 3.131 & 3.04916 & .6 & & -2.61386 & 30 & & Averaged \\
\hline Benzo(a)anthracene & 1.4837 & 1.36811 & .8 & & -7.79066 & 30 & & Averaged \\
\hline Chrysene & 1.5348 & 1.14189 & .7 & & -25.60008 & 30 & & Averaged \\
\hline Benzo(b)fluoranthene & 1.8692 & 1.77275 & .7 & & -5.15996 & 30 & & Averaged \\
\hline Benzo(k)fluoranthene & 1.8387 & 1.87331 & .7 & & 1.88231 & 30 & & Averaged \\
\hline Benzo(a)pyrene & 1.4248 & 1.32805 & .7 & & -6.79043 & 30 & & Averaged \\
\hline
\end{tabular}

Page 82 of 94 
Continuing Calibration Summary

Instrument ID: MSD4.I

Data File: $\quad$ s032918.B $\backslash$ s4c2908.D

Lab Sample ID WBN180311-86.3

Quant Type ISTD
Client SDG: $\quad 446517$

Injection Date: $\quad$ 29-MAR-18 15:12

Init. Cal. Date(s) : 01-MAR-18 09:25 - 01-MAR-18 11:4

Method: s032918.BMMSD4_SIMPAHPLUS_8270D_8270C

Method Update: 01-MAR-18 12:09

\begin{tabular}{|l|r|r|r|r|r|r|r|r|r|}
\hline Compound & $\begin{array}{c}\text { AVERF / } \\
\text { Amount }\end{array}$ & $\begin{array}{c}\text { RF } \\
\text { CCV }\end{array}$ & $\begin{array}{c}\text { Nominal } \\
\text { CCV }\end{array}$ & Min RF & $\begin{array}{c}\text { RF } \\
\text { Q }\end{array}$ & $\begin{array}{c}\text { \%D / } \\
\text { \%Drift }\end{array}$ & $\begin{array}{c}\text { Max } \\
\text { Drift } \\
\text { Q }\end{array}$ & $\begin{array}{c}\text { Curve } \\
\text { Type }\end{array}$ \\
\hline S 5-alpha-Androstane & 0.1302 & 0.14082 & & .01 & & 8.15668 & 20 & & Averaged \\
\hline Naphthalene & 1.1278 & 1.0773 & .7 & & -4.47774 & 20 & & Averaged \\
\hline Acenaphthene & 1.5198 & 1.52008 & .9 & & 0.01842 & 20 & & Averaged \\
\hline Fluorene & 1.5512 & 1.65894 & .9 & & 6.94559 & 20 & & Averaged \\
\hline Anthracene & 1.1992 & 1.17774 & .7 & & -1.78953 & 20 & & Averaged \\
\hline Fluoranthene & 1.0959 & 1.18478 & .6 & & 8.11023 & 20 & & Averaged \\
\hline Pyrene & 3.131 & 3.17886 & .6 & & 1.52859 & 20 & & Averaged \\
\hline Benzo(a)anthracene & 1.4837 & 1.62921 & .8 & & 9.80724 & 20 & & Averaged \\
\hline Chrysene & 1.5348 & 1.47564 & .7 & & -3.85457 & 20 & & Averaged \\
\hline Benzo(b)fluoranthene & 1.8692 & 1.81313 & .7 & & -2.99968 & 20 & & Averaged \\
\hline Benzo(k)fluoranthene & 1.8387 & 1.67732 & .7 & & -8.77685 & 20 & & Averaged \\
\hline Benzo(a)pyrene & 1.4248 & 1.52591 & .7 & & 7.09643 & 20 & & Averaged \\
\hline
\end{tabular}

Page 83 of 94 
Continuing Calibration Summary

Instrument ID: MSD4.I

Data File: s033018.B $\backslash$ s4c3005.D

Lab Sample ID WBN180311-86.3

Quant Type ISTD
Client SDG: $\quad 446517$

Injection Date: $\quad$ 30-MAR-18 10:20

Init. Cal. Date(s) : 01-MAR-18 09:25 - 01-MAR-18 11:4

Method: s033018.BMMSD4_SIMPAHPLUS_8270D_8270C

Method Update: 01-MAR-18 12:09

\begin{tabular}{|c|c|c|c|c|c|c|c|c|c|}
\hline Compound & $\begin{array}{c}\text { AVERF / } \\
\text { Amount }\end{array}$ & $\begin{array}{c}\mathrm{RF} \\
\mathrm{CCV}\end{array}$ & $\begin{array}{c}\text { Nominal } \\
\mathrm{CCV}\end{array}$ & Min RF & $\begin{array}{c}\text { RF } \\
\text { Q }\end{array}$ & $\begin{array}{c}\text { \%D / } \\
\text { \%Drift }\end{array}$ & Max & $\begin{array}{c}\text { Drift } \\
\text { Q }\end{array}$ & $\begin{array}{c}\text { Curve } \\
\text { Type }\end{array}$ \\
\hline S5-alpha-Androstane & 0.1302 & 0.12923 & & .01 & & -0.74501 & 20 & & Averaged \\
\hline Naphthalene & 1.1278 & 1.08447 & & .7 & & -3.84199 & 20 & & Averaged \\
\hline Acenaphthene & 1.5198 & 1.4859 & & .9 & & -2.23056 & 20 & & Averaged \\
\hline Fluorene & 1.5512 & 1.69419 & & .9 & & 9.21802 & 20 & & Averaged \\
\hline Anthracene & 1.1992 & 1.23301 & & .7 & & 2.81938 & 20 & & Averaged \\
\hline Fluoranthene & 1.0959 & 1.2305 & & .6 & & 12.28214 & 20 & & Averaged \\
\hline Pyrene & 3.131 & 2.53187 & & .6 & & -19.13542 & 20 & & Averaged \\
\hline Benzo(a)anthracene & 1.4837 & 1.66181 & & .8 & & 12.00445 & 20 & & Averaged \\
\hline Chrysene & 1.5348 & 1.75032 & & .7 & & 14.04222 & 20 & & Averaged \\
\hline Benzo(b)fluoranthene & 1.8692 & 1.80649 & & .7 & & -3.35491 & 20 & & Averaged \\
\hline Benzo(k)fluoranthene & 1.8387 & 1.71277 & & .7 & & -6.84886 & 20 & & Averaged \\
\hline Benzo(a)pyrene & 1.4248 & 1.57181 & & .7 & & 10.31794 & 20 & & Averaged \\
\hline
\end{tabular}




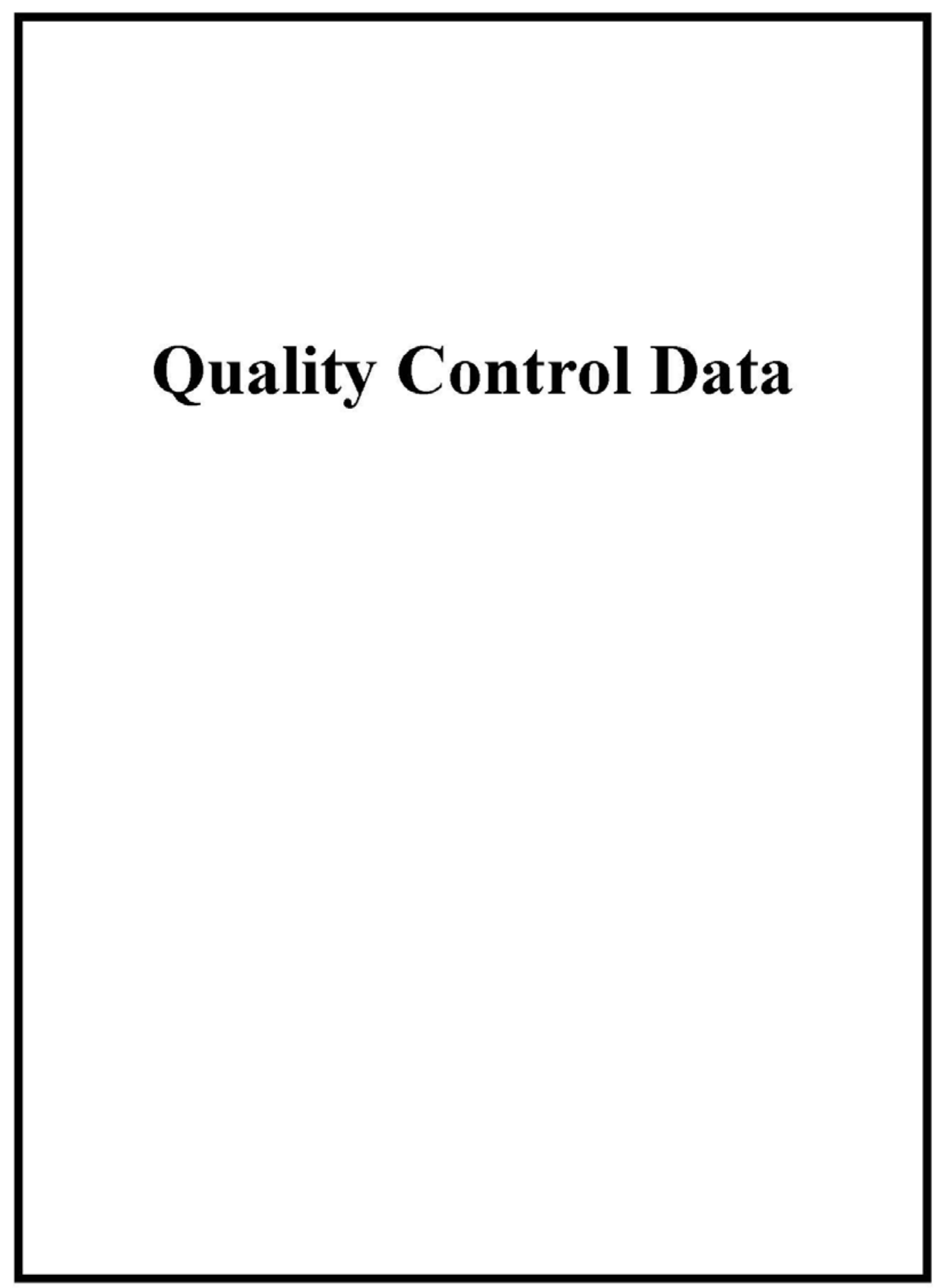

Page 85 of 94 


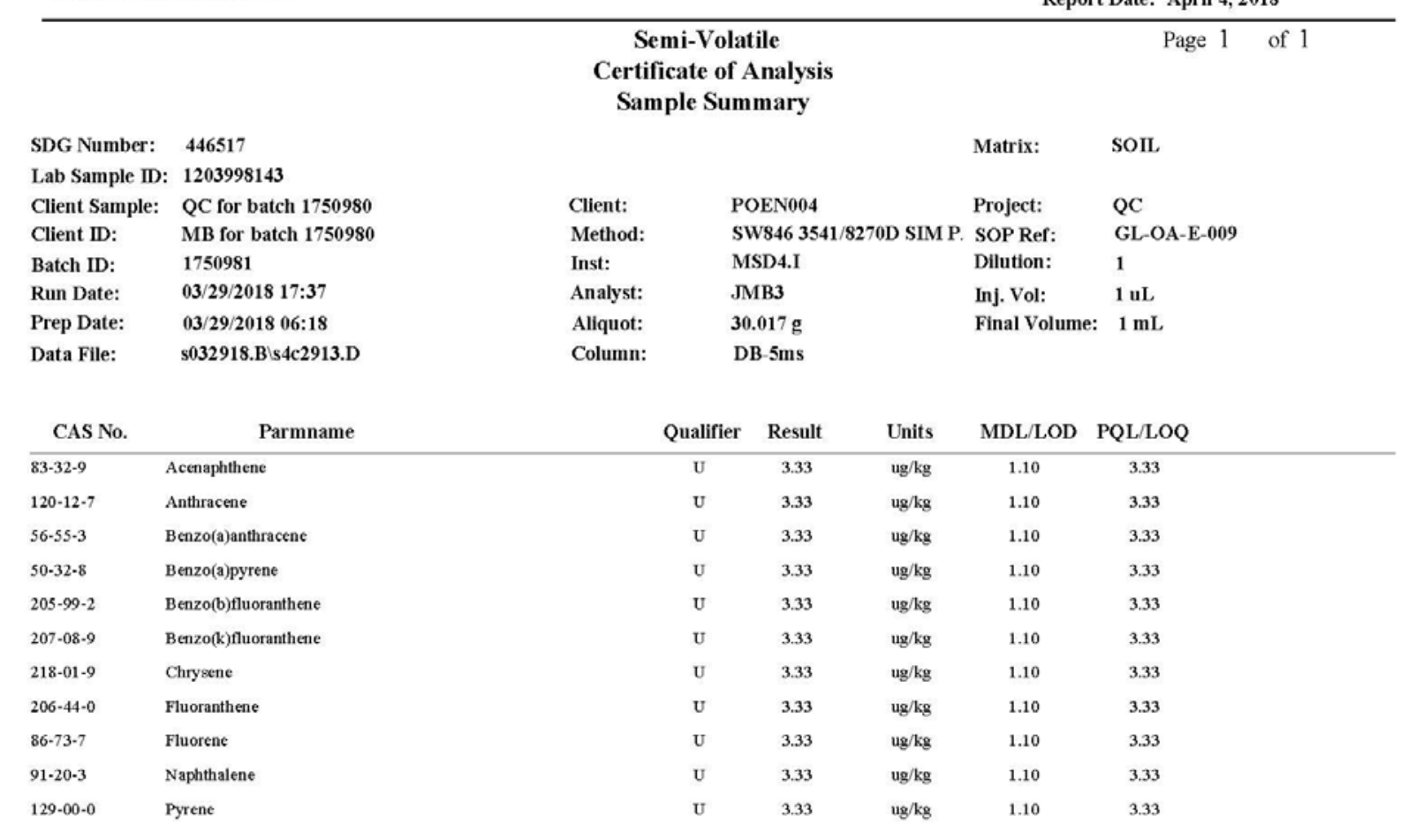




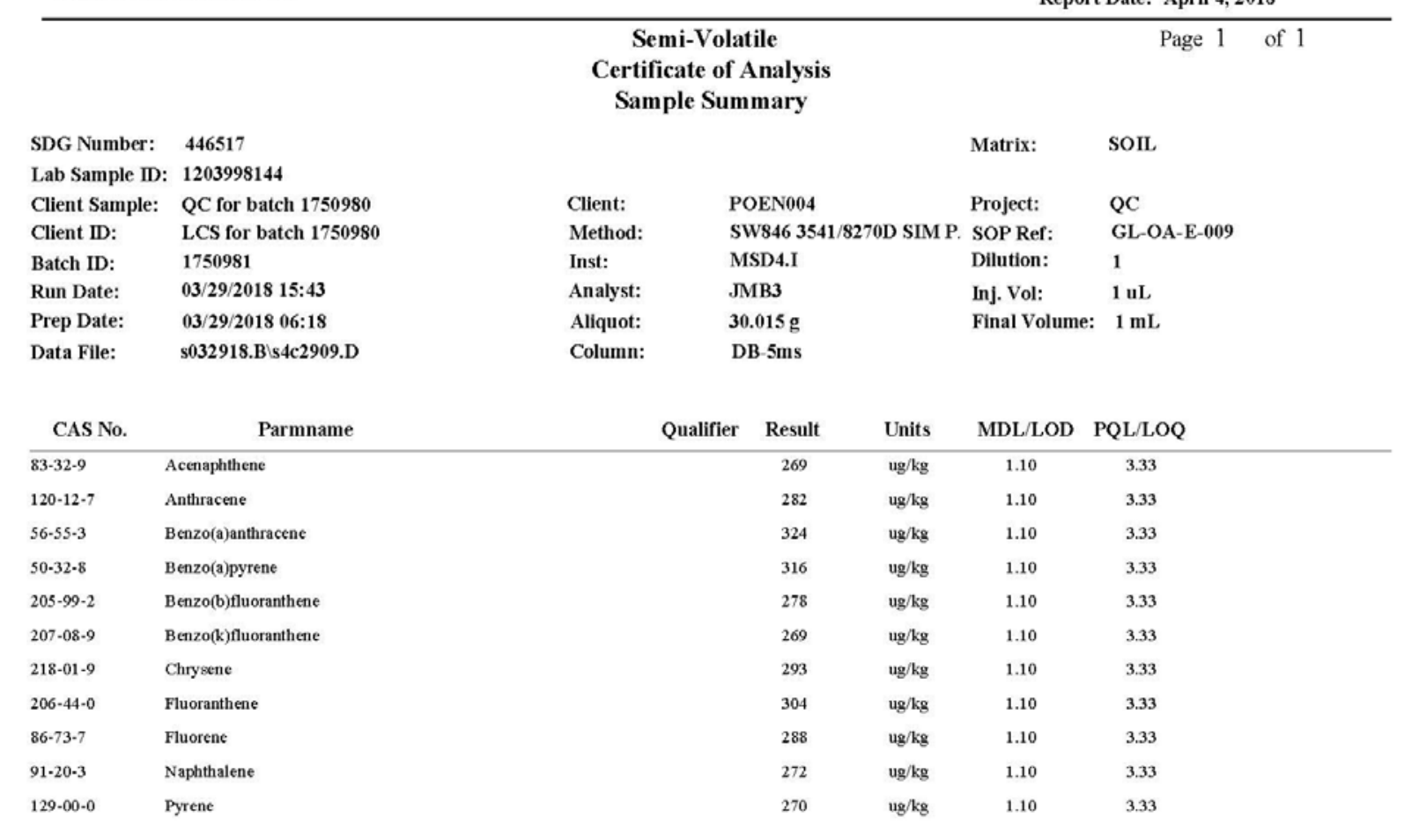




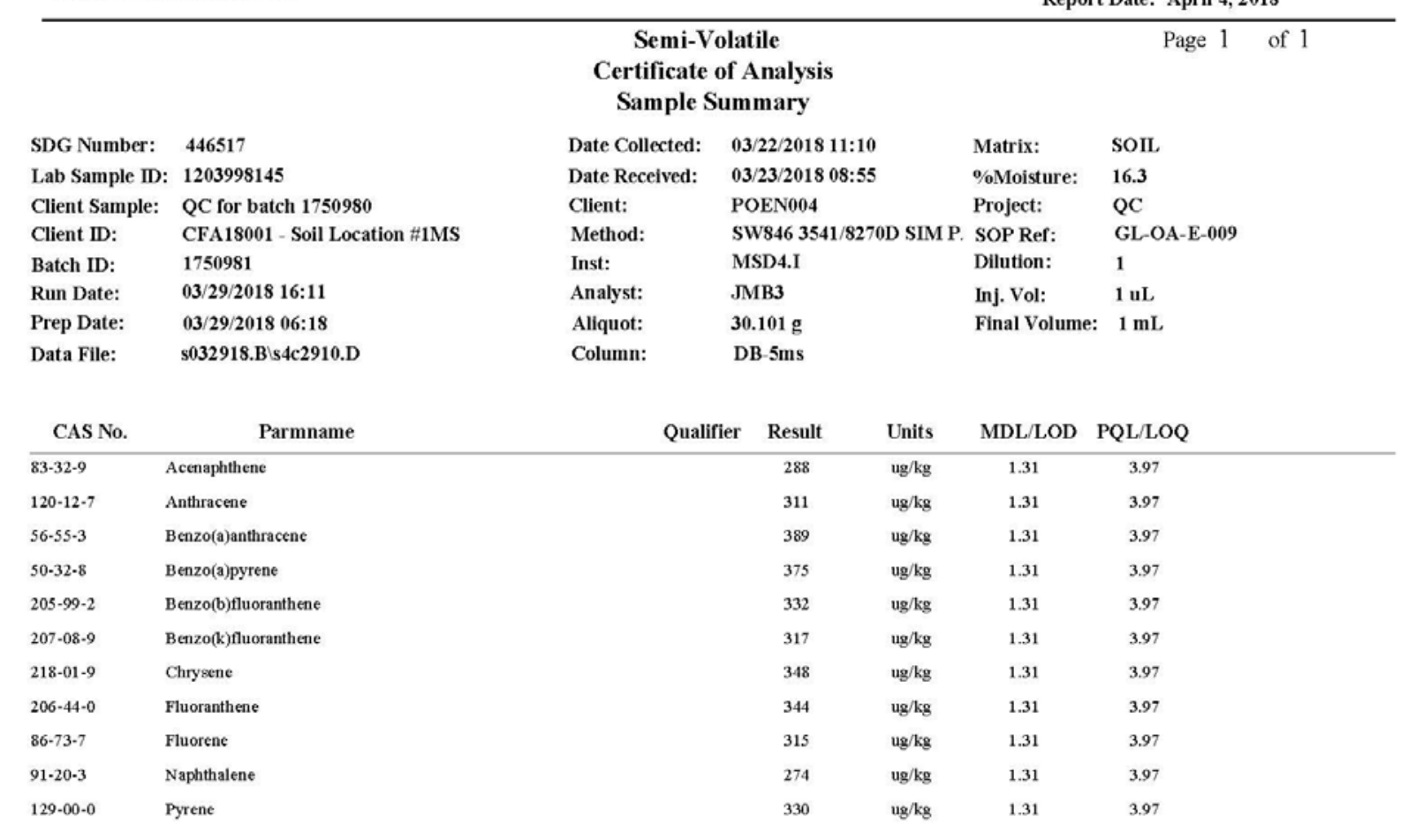




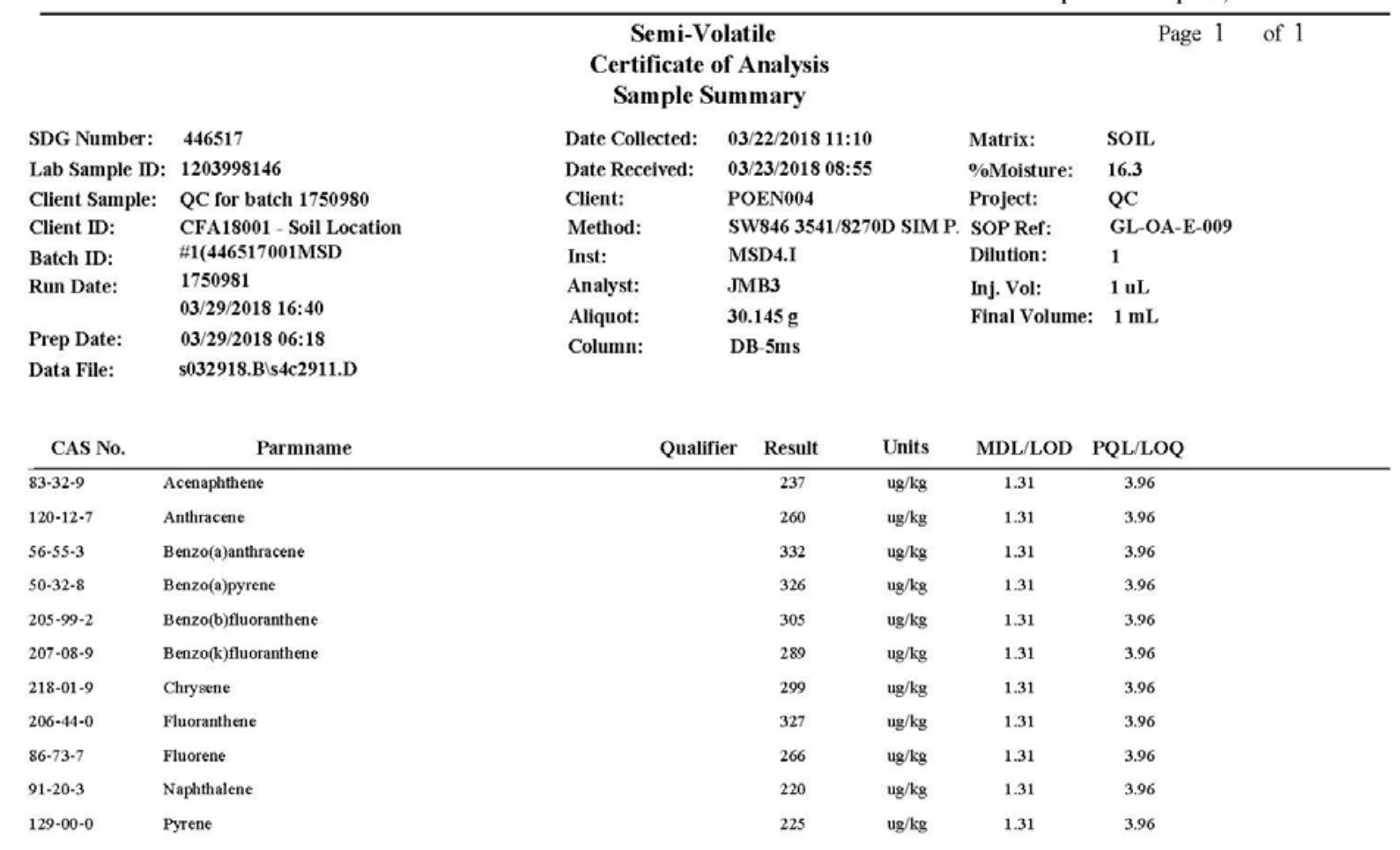




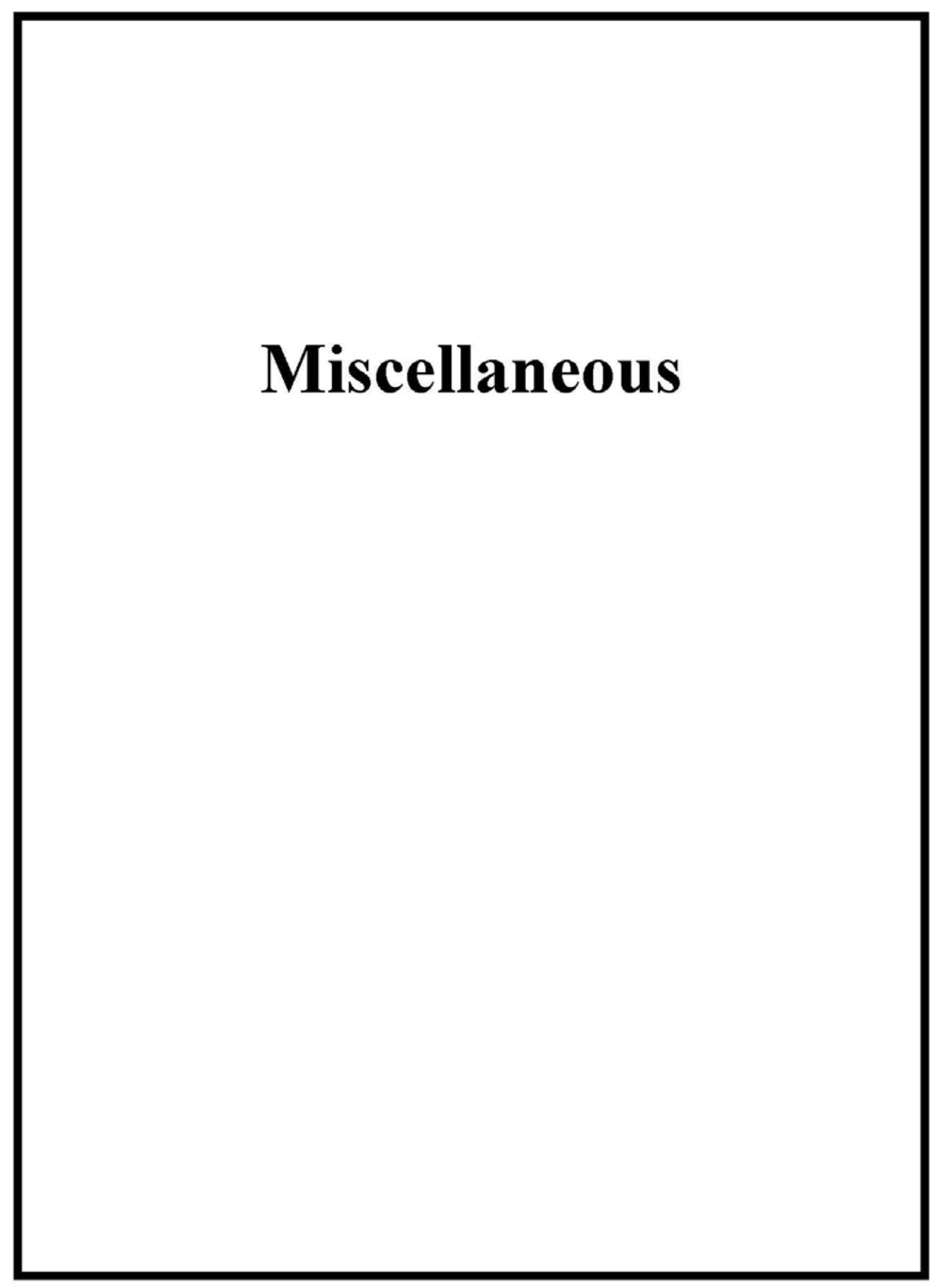

Page 90 of 94 
Prep Logbook

Automated Soxhlet Extraction

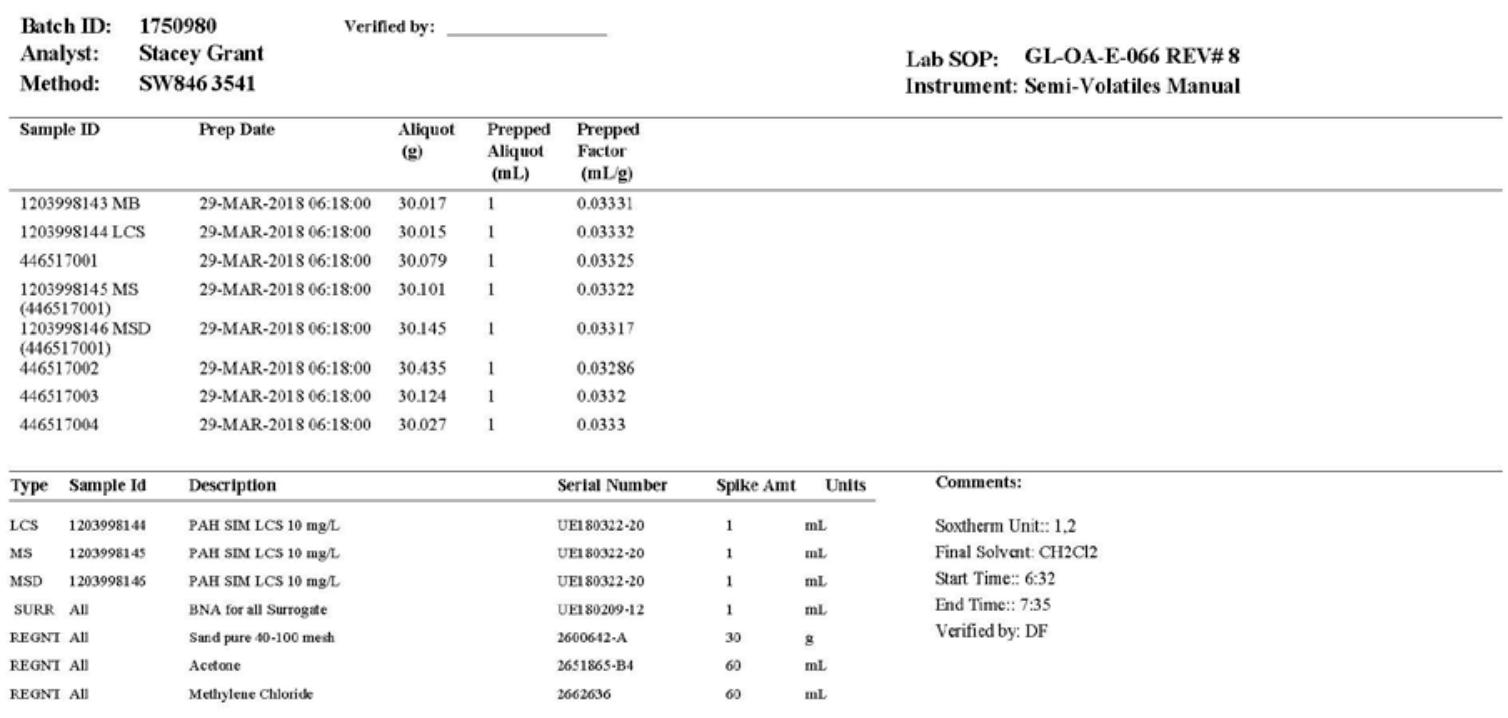




\section{GEL ORGANIC RUN LOG}

$\stackrel{\circ}{\perp}$

$$
\text { DATE: } 1 \text {-Mar-18 }
$$

Multiplier Voltage: 1941 Internal Std ID: UBN171221-01.1

Internal Std ID: WBN180209-99 (SIM) Solvent Reference ID: 2620130

\section{ORGANIC RUN LOG - INSTRUMENT ID\#MSD4}

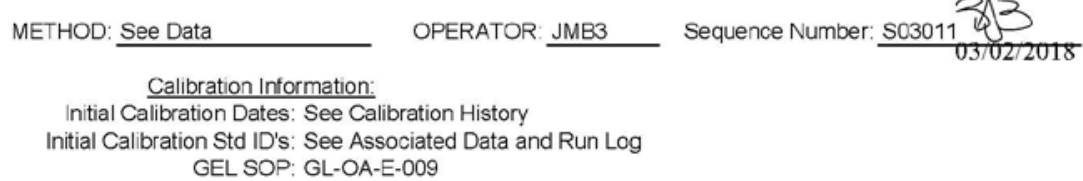
GEL SOP: GL-OA-E-009

\begin{tabular}{|c|c|c|c|c|c|c|c|c|}
\hline Analysis & & & & & Dil. & $\overline{A S}$ & Analyst & Comments \\
\hline Date Time & Data File & Lab Sample ID & Client & Batch \# & Factor & Slot \# & & \\
\hline $03 / 01 / 201809: 09$ & s4c0101.D & WBN180108-99 & DFTPP & DFTPP & 1 & 1 & JMB3 & $\sqrt{1}$ \\
\hline 03/01/2018 09:25 & $\mathrm{s} 4 \mathrm{c} 0102 . \mathrm{D}$ & WBN180209-88 & SIM-1 & ICAL & 1 & 2 & JMB3 & v \\
\hline $03 / 01 / 201809: 53$ & S4C0103.D & WBN180209-86 & SIM-3 & ICAL & 1 & 3 & JMB3 & v \\
\hline $03 / 01 / 2018$ 10:21 & S4c0104.D & WBN180209-85 & SIM-4 & ICAL & 1 & 4 & JMB3 & v \\
\hline $03 / 01 / 201810: 49$ & S4c0105.D & WBN180209-83 & SIM-6 & ICAL & 1 & 5 & JMB3 & V IS1: 35355 \\
\hline $03 / 01 / 201811: 17$ & s4c0106.D & WBN180209-82 & SIM-7 & ICAL & 1 & 6 & JMB3 & v \\
\hline $03 / 01 / 201811: 46$ & s4c0107.D & WBN180209-81 & SIM-8 & ICAL & 1 & 7 & JMB3 & V \\
\hline $03 / 01 / 201812: 16$ & S4c0108.D & WBN180301-89 & SIM-ICV & $\mathrm{ICV}$ & 1 & 8 & JMB3 & v \\
\hline $03 / 01 / 201812: 45$ & s4c0109.D & 1203979323 & MB & 1742417 & 1 & 9 & JMB3 & Report: SIM 3541 \\
\hline $03 / 01 / 201813: 40$ & S4c0110.D & 1203979324 & LCS & 1742417 & 1 & 10 & JMB3 & Report: SIM 3541 \\
\hline $03 / 01 / 201814: 08$ & S4c0111.D & 444392002 & QCQA(1/2MDL) & 1742417 & 1 & 11 & JMB3 & Report: SIM 3541 \\
\hline $03 / 01 / 201814: 36$ & s4c0112.D & 444392003 & QCQA(MDL) & 1742417 & 1 & 12 & JMB3 & Report: SIM 3541 \\
\hline $03 / 01 / 201815: 05$ & s4c0113.D & 444392004 & QCQA(LOD) & 1742417 & 1 & 13 & JMB3 & Report: SIM 3541 \\
\hline $03 / 01 / 201815: 33$ & s4c0114.D & 444392005 & QCQA(LOQ) & 1742417 & 1 & 14 & JMB3 & Report: SIM 3541 \\
\hline $03 / 01 / 201816: 01$ & s4c0115.D & 1203979402 & MB & 1742457 & 1 & 15 & JMB3 & Report: SIM 3510 \\
\hline $03 / 01 / 201816: 29$ & s4c0116.D & 1203979403 & LCS & 1742457 & 1 & 16 & JMB3 & Report: SIM 3510 \\
\hline $03 / 01 / 201816: 57$ & s4c0117.D & 444318002 & QCQA(1/2MDL) & 1742457 & 1 & 17 & JMB3 & Report: SIM 3510 \\
\hline $03 / 01 / 201817: 25$ & s4c0118.D & 444318003 & QCQA(MDL) & 1742457 & 1 & 18 & JMB3 & Report: SIM 3510 \\
\hline $03 / 01 / 201818: 21$ & s4c0120.D & 444318005 & QCQA $(L O Q)$ & 1742457 & 1 & 20 & JMB3 & Report: SIM 3510 \\
\hline $03 / 01 / 201818: 49$ & $s 4 c 0121 . D$ & WBN180209-83 & SIM-CCV & $\mathrm{CCV}$ & 1 & 21 & JMB3 & $\sqrt{ }$ \\
\hline
\end{tabular}




$$
\text { DATE: 29-Mar-18 }
$$

Multiplier Voltage: 1671 Internal Std ID: UBN171221-01.2

Internal Std ID: WBN180311-99 (SIM) Solvent Reference ID: $\frac{2620130}{20}$
METHOD: See Data

OPERATOR: JMB3

\begin{tabular}{|c|c|c|c|c|c|c|c|c|}
\hline Analysis & & & & & Dil. & $\overline{A S}$ & Analyst & Comments \\
\hline Time & Data File & Lab Sample ID & Client & Batch \# & Factor & Slot\# & & \\
\hline $03 / 29 / 201810: 49$ & $\mathrm{~s} 4 \mathrm{c} 2901 . \mathrm{D}$ & RINSE & & & & 100 & JMB3 & DUSE \\
\hline $03 / 29 / 201811: 04$ & $\mathrm{~s} 4 \mathrm{c} 2902 . \mathrm{D}$ & RINSE & & & & 100 & JMB3 & DUSE \\
\hline $03 / 29 / 201811: 19$ & $s 4 c 2903 . D$ & WBN180108-99 & DFTPP & DFTPP & 1 & 1 & JMB3 & DUSE: passed \\
\hline $03 / 29 / 201811: 34$ & s4c2904.D & WBN180220-05.2 & M-CCV & $\mathrm{CCV}$ & 1 & 2 & JMB3 & DUSE: HCCPD, acids low.$->$ performed maintenance \\
\hline $03 / 29 / 201812: 43$ & $s 402905 . D$ & WBN180108-99 & DFTPP & DFTPP & 1 & 1 & JMB3 & DUSE: no PCP \\
\hline $03 / 29 / 201812: 59$ & $s 4 c 2906 . D$ & WBN180220-05.2 & M-CCV & $\mathrm{CCV}$ & 1 & 2 & JMB3 & DUSE: acids low $\rightarrow>$ performed maintenance \\
\hline $03 / 29 / 201814: 56$ & s4c2907.D & WBN180108-99 & DFTPP & DFTPP & 1 & 1 & JMB3 & i no PCP, not required for SIM PAH's \\
\hline $03 / 29 / 201815: 12$ & $s 402908 . D$ & WBN180311-86.3 & S-CCV & $\mathrm{CCV}$ & 1 & 3 & JMB3 & IS1: 44686 \\
\hline $03 / 29 / 201815: 43$ & $S 4 c 2909 . D$ & 1203998144 & LCS & 1750981 & 1 & 4 & JMB3 & Report \\
\hline $03 / 29 / 201816: 11$ & $s 4 c 2910 . D$ & 1203998145 & MS & 1750981 & 1 & 5 & JMB3 & Report \\
\hline $03 / 29 / 201816: 40$ & s4c2911.D & 1203998146 & MSD & 1750981 & 1 & 6 & JMB3 & Report \\
\hline $03 / 29 / 201817: 08$ & $s 4 c 2912 . D$ & 1203998158 & LCS & 1750987 & 1 & 7 & JMB3 & Report \\
\hline $03 / 29 / 201817: 37$ & $\mathrm{~s} 4 \mathrm{c} 2913 . \mathrm{D}$ & 1203998143 & $\mathrm{MB}$ & 1750981 & 1 & 8 & JMB3 & Report \\
\hline $03 / 29 / 201818: 05$ & s4c2914.D & 1203998157 & MB & 1750987 & 1 & 9 & JMB3 & DUSE: failed ISTD - see Ir S4c3006 \\
\hline $03 / 29 / 201818: 34$ & $\mathrm{~s} 4 \mathrm{c} 2915 . \mathrm{D}$ & 446517001 & POEN & 1750981 & 1 & 10 & JMB3 & Report \\
\hline $03 / 29 / 201819: 02$ & $\mathrm{~s} 4 \mathrm{C} 2916 . \mathrm{D}$ & 446517002 & POEN & 1750981 & 1 & 11 & JMB3 & DUSE: failed ISTD - see Ir S4c3007 \\
\hline $03 / 29 / 201819: 31$ & s4c2917.D & 446517003 & POEN & 1750981 & 1 & 12 & JMB3 & Report \\
\hline $03 / 29 / 201819: 59$ & s4c2918.D & 446517004 & POEN & 1750981 & 1 & 13 & JMB3 & Report \\
\hline $03 / 29 / 201820: 28$ & $s 4 c 2919 . D$ & 446352002 & SAME & 1750987 & 1 & 14 & JMB3 & DUSE: failed surr - RX s2d0309 passed w/in hold \\
\hline $03 / 29 / 201820: 56$ & $54 c 2920 . D$ & 446352003 & SAME & 1750987 & 1 & 15 & JMB3 & Report \\
\hline $03 / 29 / 201821: 24$ & $s 4 c 2921 . D$ & 446352004 & SAME & 1750987 & 1 & 16 & JMB3 & DUSE: failed ISTD - see Ir $\$ 403022$ \\
\hline $03 / 29 / 201821: 53$ & $s 4 c 2922 . D$ & 446352005 & SAME & 1750987 & 1 & 17 & JMB3 & DUSE: failed ISTD - see Ir S4c3023 \\
\hline $03 / 29 / 201822: 21$ & $s 402923 . D$ & 446352006 & SAME & 1750987 & 1 & 18 & JMB3 & DUSE: failed ISTD - see Ir s4c3024 \\
\hline $03 / 29 / 201822: 50$ & $\mathrm{~s} 4 \mathrm{c} 2924 . \mathrm{D}$ & 446352008 & SAME & 1750987 & 1 & 19 & JMB3 & DUSE: failed ISTD - see Ir s4c3025 \\
\hline $03 / 29 / 201823: 18$ & $\mathrm{~s} 4 \mathrm{c} 2925 . \mathrm{D}$ & 446352001 & SAME & 1750987 & 1 & 20 & JMB3 & Report \\
\hline $03 / 29 / 201823: 47$ & $s 4 c 2926 . \mathrm{D}$ & 1203998159 & MS & 1750987 & 1 & 21 & JMB3 & Report \\
\hline $03 / 30 / 201800: 15$ & $\mathrm{~s} 4 \mathrm{c} 2927 . \mathrm{D}$ & 1203998160 & MSD & 1750987 & 1 & 22 & JMB3 & DUSE: MSD mislabeled with MSD for Scan - see ir $\$ 4 c 3008$ \\
\hline $03 / 30 / 201800: 43$ & $s 4 c 2928 . D$ & 446352007 & SAME & 1750987 & 1 & 23 & JMB3 & DUSE: possible carryover - see rr \$4c3026 \\
\hline $03 / 30 / 201801: 12$ & $s 4 c 2929 . \mathrm{D}$ & 446352009 & SAME & 1750987 & 1 & 24 & JMB3 & Report \\
\hline $03 / 30 / 201801: 40$ & $\mathrm{~s} 4 \mathrm{c} 2930 . \mathrm{D}$ & 446352010 & SAME & 1750987 & 1 & 25 & JMB3 & Report \\
\hline $03 / 30 / 201802: 08$ & $\mathrm{~s} 4 \mathrm{c} 2931 . \mathrm{D}$ & 446352011 & SAME & 1750987 & 1 & 26 & JMB3 & Report \\
\hline $03 / 30 / 201802: 36$ & $\mathrm{~s} 4 \mathrm{c} 2932 . \mathrm{D}$ & WBN180108-99 & DFTPP & DFTPP & 1 & 1 & JMB3 & DUSE: post sequence screen - breakdown $>20 \%$ \\
\hline
\end{tabular}

Calibration Information:

Initial Calibration Dates: See Calibration History

nitial Calibration Std ID's: See Associated Data and Run Log GEL SOP: GL-OA-E-009 
GEL ORGANIC RUN LOG

$\stackrel{\circ}{\perp}$

DATE: 30 -Mar-18

Multiplier Voltage: 1671

Internal Std ID: UBN171221-01.2

Internal Std ID WBN171226-99 (SIM)

Solvent Reference ID: $\underline{2620130}$
ORGANIC RUN LOG - INSTRUMENT ID\#MSD4

METHOD: See Data

OPERATOR: JMB3

Sequence Number: S033018.B

Calibration Information:

Initial Calibration Dates: See Calibration History

Initial Calibration Std ID's: See Associated Data and Run Log GEL SOP: GL-OA-E-009

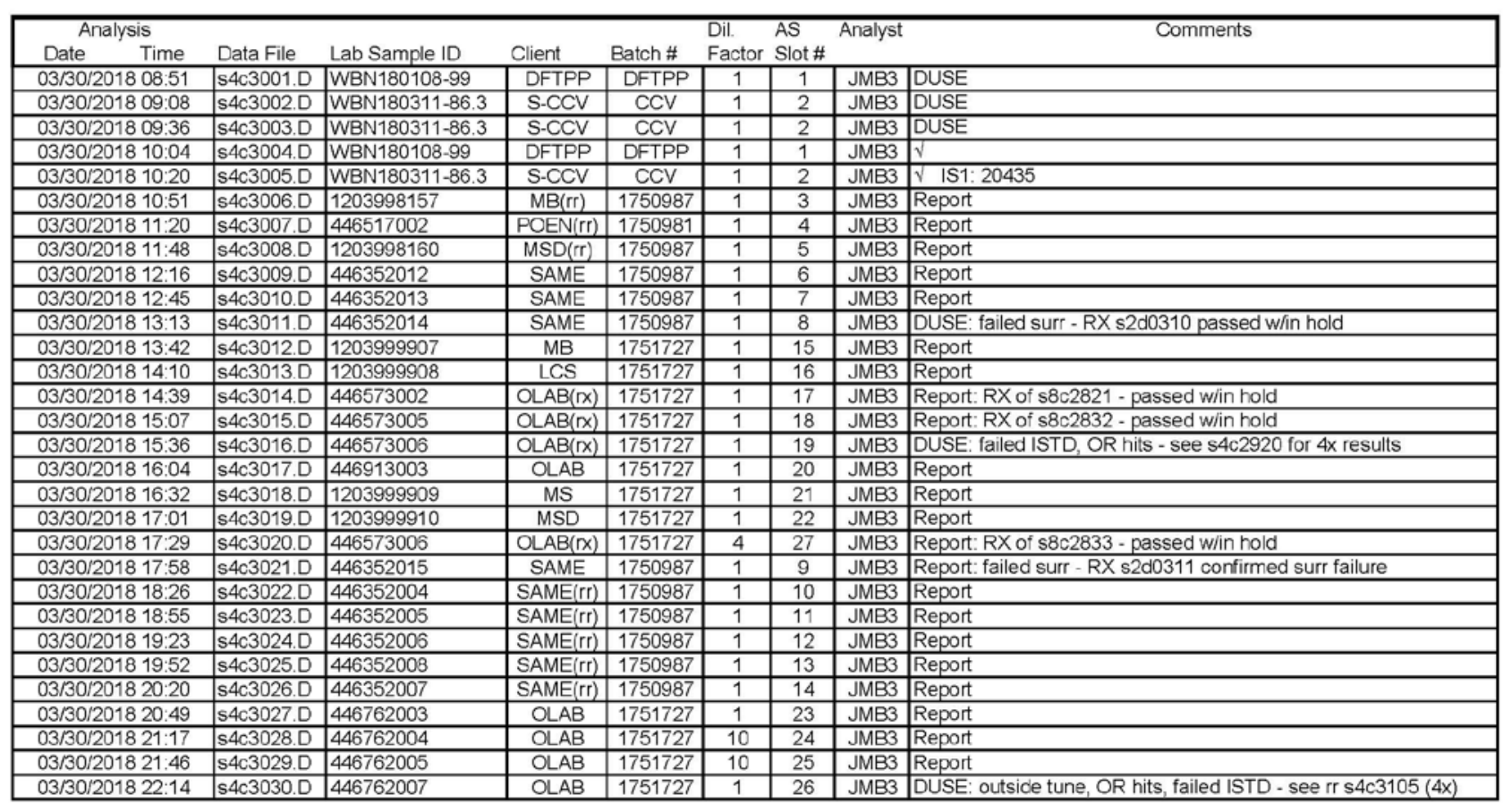

Page: 105 


\title{
Appendix $F$ \\ Idaho Risk Evaluation Manual for Petroleum Releases
}

\author{
Idaho \\ Risk Evaluation Manual \\ for Petroleum Releases
}

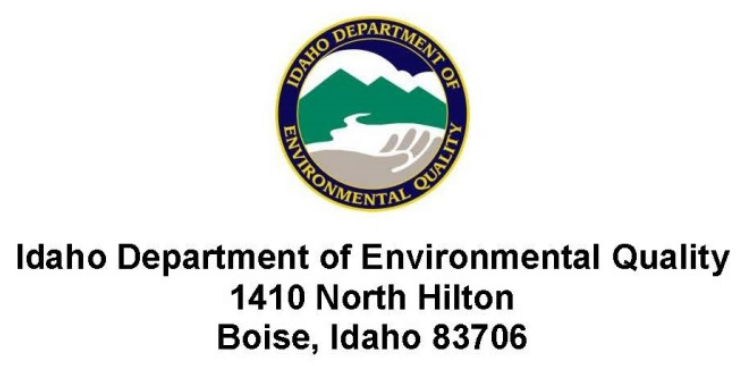

August 2012 
Table 2 lists the screening levels for unrestricted use. For comparison, Table 2 provides the riskbased concentrations in soil and groundwater for all the pathways and ROE listed above. The screening level values incorporated in the Rule are indicated in bold.

Because of the methods and assumptions used in the development of the screening levels and the current limitations of laboratory analytical methods, the calculated screening levels may be lower than the practical quantitation limit reported by a laboratory for selected chemicals. In these situations, site-specific review by DEQ will be required based on the criteria provided in Section 500 of the Rule and Appendix K.

Table 2. Screening Level Concentrations for Soil, Groundwater, and Soil Vapor

\begin{tabular}{|c|c|c|c|c|c|c|c|}
\hline \multirow[b]{3}{*}{ CHEMICAL } & \multicolumn{3}{|c|}{$\begin{array}{c}\text { SOIL } \\
(\mathrm{mg} / \mathrm{kg})\end{array}$} & \multirow{2}{*}{\multicolumn{2}{|c|}{$\begin{array}{l}\text { GROUNDWATER } \\
(\mathrm{mg} / \mathrm{L})\end{array}$}} & \multicolumn{2}{|c|}{$\begin{array}{c}\text { DEEP SOIL VAPOR } \\
\text { (ug/m3) } \\
\text { (>3-5 feet bgs) }\end{array}$} \\
\hline & & & & & & \multirow{2}{*}{$\begin{array}{c}\text { Unrestricted } \\
\text { Use } \\
\text { Vapor } \\
\text { Intrusion } \\
\end{array}$} & \multirow{2}{*}{$\begin{array}{c}\begin{array}{c}\text { Commercial/ } \\
\text { Industrial }\end{array} \\
\text { Vapor } \\
\text { Intrusion }\end{array}$} \\
\hline & \begin{tabular}{|c|} 
Vapor \\
Intrusion
\end{tabular} & $\begin{array}{c}\text { Direct } \\
\text { Contact }\end{array}$ & $\begin{array}{c}\text { Groundwater } \\
\text { Protection }\end{array}$ & $\begin{array}{l}\text { Vapor } \\
\text { Intrusion }\end{array}$ & Ingestion & & \\
\hline Benzene & 0.08 & 8.3 & 0.025 & 0.044 & 0.005 & 31 & 160 \\
\hline Toluene & 1300 & 7930 & 6.6 & 340 & 1 & 520000 & 2200000 \\
\hline Ethylbenzene & 0.25 & 39 & 7.4 & 0.05 & 0.700 & 97 & 490 \\
\hline Xylenes & 27 & 6170 & 91 & 8.7 & 10 & 10000 & 44000 \\
\hline Naphthalene & 0.12 & 44 & 9.2 & 0.07 & 0.73 & 7 & 36 \\
\hline MTBE & 2.4 & 340 & 0.08 & 6.8 & 0.04 & 940 & 4700 \\
\hline 1,2-Dichloroethane & 0.02 & 3.7 & 0.013 & 0.03 & 0.005 & 9 & 47 \\
\hline Ethylene Dibromide & 0.001 & 0.27 & 0.00014 & 0.004 & 0.00005 & 0.4 & 2 \\
\hline Acenaphthene & NA & 4470 & 200 & NA & 2.2 & NA & NA \\
\hline Anthracene & NA & 22300 & 3200 & NA & 11 & NA & NA \\
\hline Benz(a)anthracene & NA & 0.19 & 0.09 & NA & 0.00003 & NA & NA \\
\hline Benzo(a)pyrene & NA & 0.02 & 2.1 & NA & 0.0002 & NA & NA \\
\hline Benzo(b)fluoranthene & NA & 0.19 & 0.31 & NA & 0.00003 & NA & NA \\
\hline Benzo(k)fluoranthene & NA & 1.9 & 3.1 & NA & 0.0003 & NA & NA \\
\hline Chrysene & NA & 19 & 9.5 & NA & 0.003 & NA & NA \\
\hline Fluoranthene & NA & 2970 & 1400 & NA & 1.5 & NA & NA \\
\hline Fluorene & NA & 2970 & 240 & NA & 1.5 & NA & NA \\
\hline Pyrene & NA & 2230 & 1000 & NA & 1.1 & NA & NA \\
\hline
\end{tabular}

Values in bold are current screening level values specified in the Rule. Screening level values for deep soil vapor are equivalent to EPA Regional Screening Levels (EPA, 2012) for residential and industrial ambient air divided by an attenuation factor of 0.01 . NA: not applicable because the chemical does not meet EPA volatility criteria or does not have a Regional Screening Level for ambient air. 